\title{
Geometric Structures on Spaces of Weighted Submanifolds
}

\author{
Brian LEE
}

Department of Mathematics, University of Toronto, Toronto, ON M5S $2 E_{4}$ Canada

E-mail: brianlee@math.toronto.edu

Received May 31, 2009, in final form October 25, 2009; Published online November 02, 2009 doi:10.3842/SIGMA.2009.099

\begin{abstract}
In this paper we use a diffeo-geometric framework based on manifolds that are locally modeled on "convenient" vector spaces to study the geometry of some infinite dimensional spaces. Given a finite dimensional symplectic manifold $(M, \omega)$, we construct a weak symplectic structure on each leaf $\mathbf{I}_{w}$ of a foliation of the space of compact oriented isotropic submanifolds in $M$ equipped with top degree forms of total measure 1 . These forms are called weightings and such manifolds are said to be weighted. We show that this symplectic structure on the particular leaves consisting of weighted Lagrangian submanifolds is equivalent to a heuristic weak symplectic structure of Weinstein [Adv. Math. 82 (1990), 133-159]. When the weightings are positive, these symplectic spaces are symplectomorphic to reductions of a weak symplectic structure of Donaldson [Asian J. Math. 3 (1999), 1-15] on the space of embeddings of a fixed compact oriented manifold into $M$. When $M$ is compact, by generalizing a moment map of Weinstein we construct a symplectomorphism of each leaf $\mathbf{I}_{w}$ consisting of positive weighted isotropic submanifolds onto a coadjoint orbit of the group of Hamiltonian symplectomorphisms of $M$ equipped with the Kirillov-Kostant-Souriau symplectic structure. After defining notions of Poisson algebras and Poisson manifolds, we prove that each space $\mathbf{I}_{w}$ can also be identified with a symplectic leaf of a Poisson structure. Finally, we discuss a kinematic description of spaces of weighted submanifolds.
\end{abstract}

Key words: infinite dimensional manifolds; weakly symplectic structures; convenient vector spaces; Lagrangian submanifolds; isodrastic foliation

2000 Mathematics Subject Classification: 58B99

\section{Introduction}

In the same way that finite dimensional manifolds are locally modeled on $\mathbb{R}^{n}$, many collections of geometric objects can be viewed as infinite dimensional manifolds locally modeled on interesting geometric spaces. For example, if $N$ and $M$ are smooth manifolds then the following local models are known:

\begin{tabular}{|l||l|}
\hline Collection $\mathcal{M}$ & Modeling Space at $x \in \mathcal{M}$ \\
\hline \hline diffeomorphisms of $N$ & vector fields on $N$ \\
\hline \hline Riemannian metrics on $N$ & symmetric 2-tensors on $N$ \\
\hline \hline smooth maps from $N$ to $M$ & sections of $x^{*} T M$ \\
\hline \hline Lagrangian submanifolds & closed 1-forms on $x$ \\
\hline
\end{tabular}

These local models represent certain choices, as many geometric structures coincident in finite dimensions diverge in infinite dimensions. For example, there are typically more derivations than equivalence classes of paths; there are many ways to define the dual of a tangent space; there may fail to exist holomorphic charts even when the Nijenhuis tensor vanishes, etc. Accordingly, there are many frameworks available to study differential geometric structures in infinite dimensions. 
Depending on the problem, one might choose to work with Fréchet manifolds [7], Diffeology [15], Differential Spaces [13], the Global Analysis framework of Frölicher, Kriegl and Michor [6, 9], etc., or just choose to work heuristically.

Once a framework has been chosen, and a local model identified, the geometry of a collection $\mathcal{M}$ can be explored using the following correspondence: structures inherent to objects in $\mathcal{M}$ induce global structures on $\mathcal{M}$. For example, if $N$ and $M$ are as above then

- the set of Riemannian metrics on $N$ inherits weak Riemannian structures (Ebin 1970 [5], Smolentzev 1994 [14]);

- if $M$ is symplectic and $L \rightarrow M$ is a prequantization line bundle, then the space of sections $\Gamma(L)$ inherits a weak symplectic structure (Donaldson 2001 [4]);

- the set of embeddings of $N$ into $M$ is the total space of a principal fiber bundle with structure group $\operatorname{Diff}(N)$, the diffeomorphisms of $N$, and base the set of submanifolds of $M$ diffeomorphic to $N$ (Binz, Fischer 1981 [1]).

In this paper we study a particularly interesting example of this phenomenon involving Lagrangian submanifolds equipped with certain measures. From the very beginning, we study these objects in the "Convenient Setup" of Frölicher, Kriegl, and Michor (see [9]).

The starting point for this framework is the definition of smooth curves in locally convex spaces called convenient vector spaces. Once the smooth curves have been specified, smooth maps between convenient vector spaces can be defined as maps which send smooth curves to smooth curves. Smooth manifolds then are defined as sets that can be modeled on convenient vector spaces via charts, whose transition functions are smooth. Once the appropriate notions of smoothness are specified, objects in differential geometry are defined by choosing how to generalize finite dimensional constructions to infinite dimensions (e.g. Lie groups, principal $G$ bundles, vector fields, differential forms, etc.) An important feature of this approach is that the modeling space $E_{U}$ for each chart $(\varphi, U)$ can be different for different chart neighbourhoods $U$. This differs from the usual description of finite dimensional manifolds which are always modeled on the same vector space $\mathbb{R}^{n}$. This flexibility is useful in describing the local structure of many infinite dimensional manifolds, including the collection of Lagrangian submanifolds in a symplectic manifold.

In 1990 Alan Weinstein [23] introduced a foliation $\mathcal{F}$ of the space of Lagrangian submanifolds in a fixed symplectic manifold $(M, \omega)$. A leaf of $\mathcal{F}$ consists of Lagrangian submanifolds that can be joined by flowing along Hamiltonian vector fields. $\mathcal{F}$ lifts to a foliation $\mathcal{F}_{w}$ of the space of pairs $(L, \rho)$, where $L$ is a Lagrangian submanifold in $M$ equipped with a smooth density $\rho$ of total measure 1. Weinstein called such pairs weighted Lagrangian submanifolds and leaves of $\mathcal{F}$ and $\mathcal{F}_{w}$ isodrasts. He showed that each leaf $\mathbf{I}_{w}$ of $\mathcal{F}_{w}$ can be given a weakly nondegenerate symplectic structure $\Omega^{W}$. He also showed that the leaves consisting of Lagrangian submanifolds equipped with positive densities can be identified with coadjoint orbits of the group of Hamiltonian symplectomorphisms. All of these constructions were done on a heuristic level.

Instead of starting with the Lagrangian submanifolds directly, we instead begin by showing that the set of Lagrangian embeddings of a fixed compact oriented manifold $L_{0}$ into $M$ is the total space of a principal fiber bundle with structure group Diff $+\left(L_{0}\right)$, the orientation preserving diffeomorphisms of $L_{0}$. The base $\operatorname{Lag}(M)$ is naturally identified with the space of oriented Lagrangian submanifolds in $M$ diffeomorphic to $L_{0}$. We define a foliation $\mathcal{E}$ of the total space which descends to the isodrastic foliation $\mathcal{F}$ of the space of Lagrangian submanifolds. Similarly, the product of the space of Lagrangian embeddings with the space of top degree forms on $L_{0}$ that integrate to 1 is the total space of a principal Diff ${ }_{+}\left(L_{0}\right)$ bundle. The base of this bundle can be identified with the set of pairs $(L, \rho)$, where $L$ is an oriented Lagrangian submanifold in $M$ diffeomorphic to $L_{0}$ equipped with a top degree form $\rho$ (not necessarily non-vanishing) 
satisfying $\int_{L} \rho=1$. The foliation $\mathcal{E}$ gives a foliation $\mathcal{E}_{w}$ of the total space that descends to the isodrastic foliation $\mathcal{F}_{w}$ of the base. We define a basic 2 -form $\Omega$ on the leaves of $\mathcal{E}_{w}$ which descends to a weakly nondegenerate symplectic structure on the leaves of $\mathcal{F}_{w}$. We then show that the tangent spaces to the space of pairs $(L, \rho)$ in the "Convenient Setup" can be identified with the tangent spaces in Weinstein's heuristic construction, and that $\Omega$ corresponds to $\Omega^{W}$. In this way we make rigourous Weinstein's original construction.

The set of pairs $(L, \rho)$ consisting of Lagrangian submanifolds equipped with volume forms of total measure 1 is an open subset of the set of all weighted Lagrangian submanifolds. The leaves of $\mathcal{F}_{w}$ in this open subset of positive weighted Lagrangian submanifolds inherit the symplectic structure $\Omega$ and provide a link between Weinstein's symplectic structure and a symplectic structure defined by Simon Donaldson on the space of smooth mappings between manifolds described briefly as follows.

In 1999 Donaldson [3] heuristically wrote down a symplectic structure $\Omega^{D}$ on the space of smooth mappings $C^{\infty}\left(S_{0}, M\right)$ of a compact oriented manifold $S_{0}$, equipped with a fixed volume form $\eta_{0}$, into a symplectic manifold $(M, \omega)$. Under some topological restrictions on $\omega$ and $S_{0}$, Donaldson described a moment map $\mu$ for the $\operatorname{Diff}\left(S_{0}, \eta_{0}\right)$-action of volume preserving diffeomorphisms on $C^{\infty}\left(S_{0}, M\right)$. This $\operatorname{Diff}\left(S_{0}, \eta_{0}\right)$-action restricts to a Hamiltonian action on the space of embeddings $\operatorname{Emb}\left(S_{0}, M\right) \subset C^{\infty}\left(S_{0}, M\right)$, with respect to the restrictions of $\Omega^{D}$ and $\mu$. By a lemma of Moser, symplectic quotients of $\operatorname{Emb}\left(S_{0}, M\right)$ by $\operatorname{Diff}\left(S_{0}, \eta_{0}\right)$ can be identified with spaces of submanifolds in $M$ equipped with volume forms of fixed total measure. In fact when $S_{0}$ is half the dimension of $M$ the level surface $\mu^{-1}\{0\}$ consists of Lagrangian embeddings. This suggests that when $\eta_{0}$ has total measure 1 the symplectic quotients of $\left(\operatorname{Emb}\left(S_{0}, M\right), \Omega^{D}\right)$ should be related to the leaves of $\mathcal{F}_{w}$ consisting of positive weighted Lagrangian submanifolds.

The main result of this paper is that reductions of $\left(\operatorname{Emb}\left(S_{0}, M\right), \Omega^{D}\right)$ can be defined, in the "Convenient Setup", without any topological restrictions on $\omega$ or $S_{0}$ and that these reductions are symplectomorphic to leaves of $\mathcal{F}_{w}$ consisting of positive weighted Lagrangian submanifolds when the dimension of $S_{0}$ is half the dimension of $M$. In this way we obtain not only a rigorous formulation of Donaldson's heuristic constructions, but also a precise relationship between Weinstein's symplectic structure and Donaldson's symplectic structure. Namely, symplectic quotients of Donaldson's symplectic space can be identified with Weinstein's symplectic spaces in the particular case of leaves consisting of positive weighted Lagrangian submanifolds.

For $S_{0}$ of dimension less than or equal to half the dimension of $M$, symplectic reductions of $\left(\operatorname{Emb}\left(S_{0}, M\right), \Omega^{D}\right)$ are still well defined in the "Convenient Setup" and yield symplectic spaces consisting of positive weighted isotropic submanifolds in $M$. This suggests that the symplectic structure $\Omega$ on weighted Lagrangian submanifolds should have a generalization to weighted isotropic submanifolds. We show that indeed such a generalization exists, and that the corresponding symplectic spaces in the particular case of leaves consisting of positive weighted isotropic submanifolds are symplectomorphic to reductions of $\left(\operatorname{Emb}\left(S_{0}, M\right), \Omega^{D}\right)$. In this way we obtain a generalization of our observed relationship between Weinstein's symplectic structure and Donaldson's symplectic structure to the case of weighted isotropic submanifolds.

Our next result takes its cue from this generalization to weighted isotropic submanifolds. Namely, we show that the symplectic spaces of positive weighted isotropic submanifolds are symplectomorphic to coadjoint orbits of the group $\operatorname{Ham}(M)$ of Hamiltonian symplectomorphisms of $M$ equipped with the Kirillov-Kostant-Souriau symplectic structure. This symplectomorphism is given by a generalization of the moment map written down by Weinstein in his identification of positive weighted Lagrangian submanifolds with coadjoint orbits of $\operatorname{Ham}(M)$. The heuristic idea is that any submanifold $I$ equipped with a volume form $\rho$ can be viewed as an element of the dual of the Lie algebra of Hamiltonian vector fields via the mapping $(I, \rho) \mapsto\left(f \mapsto \int_{I} f \rho\right)$. This mapping is equivariant, injective, and hence induces a coadjoint 
orbit symplectic structure on spaces of positive weighted submanifolds that can be joined by Hamiltonian deformations.

These positive weighted isotropic submanifolds have yet another interpretation akin to leaves of Poisson manifolds in finite dimensions. Given a finite dimensional Poisson manifold $(P,\{\cdot, \cdot\})$, for each smooth function $f \in C^{\infty}(P, \mathbb{R})$ on $P$ there exists a unique vector field $X_{f}$ on $P$ satisfying $\mathrm{d} g\left(X_{f}\right)=\{f, g\}$ for all $g \in C^{\infty}(P, \mathbb{R})$. The leaves swept out by integral curves to such vector fields $X_{f}$ are symplectic manifolds. This picture can be adapted to infinite dimensions in the following sense. Given an infinite dimensional manifold $P$, for a subalgebra $\mathcal{A} \subset C^{\infty}(P, \mathbb{R})$ we define a Poisson bracket $\{\cdot, \cdot\}$ on $\mathcal{A}$ and a Poisson algebra $(\mathcal{A},\{\cdot, \cdot\})$ in the usual way. If for every $f \in \mathcal{A}$ there exists a unique vector field $X_{f}$ on $P$ satisfying $\mathrm{d} g\left(X_{f}\right)=\{f, g\}$ for all $g \in \mathcal{A}$, then the directions swept out by such vector fields on each point in $P$ define a distribution on $P$. We call maximal integral manifolds of this distribution leaves. By defining a Poisson algebra on Emb $\left(S_{0}, M\right)$, which restricts to a Poisson algebra on the space of isotropic embeddings, which descends to a Poisson algebra on the space of positive weighted isotropic submanifolds, we show that the reductions of $\left(\operatorname{Emb}\left(S_{0}, M\right), \Omega^{D}\right)$ are symplectic leaves of a Poisson structure.

As a result we arrive at three different interpretations of the symplectic spaces consisting of positive weighted isotropic submanifolds. Namely, they can be identified with reductions of the space of embeddings $\left(\operatorname{Emb}\left(S_{0}, M\right), \Omega^{D}\right)$, with coadjoint orbits of the group $\operatorname{Ham}(M)$ of Hamiltonian symplectomorphisms, and with symplectic leaves of Poisson structures.

We then take a kinematic approach to the leaves of the foliation $\mathcal{F}$ of the space of Lagrangian submanifolds to obtain a phase space symplectic structure. That is, by viewing the leaves of $\mathcal{F}$ as possible configurations for a submanifold moving in $M$, on each Lagrangian we can associate "conjugate momenta" with top degree forms that integrate to 0 . We call such pairs $(L, \chi)$ with $L$ in a leaf of $\mathcal{F}$ satisfying $\int_{L} \chi=0$ momentum weighted Lagrangian submanifolds. By writing down what should be the canonical 1-form on this set of momentum weighted Lagrangian submanifolds and calculating its exterior derivative, we obtain a weakly symplectic structure.

Finally, we apply this kinematic approach to the set of pseudo Riemannian metrics of a fixed signature on a finite dimensional manifold $N$. This collection can be viewed as a set of submanifolds by identifying each metric with its graph as a section. Weightings then can be assigned to each metric by pulling up a structure assigned to $N$. By equipping each metric in this way with a compactly supported symmetric 2 -tensor on $N$, we show that the set of all such weighted metrics has a natural exact symplectic structure.

\section{Conventions}

Unless stated otherwise, all finite dimensional manifolds are smooth, connected, and paracompact. For manifolds $M$ and $N$ and vector bundle $E \rightarrow M$, we will use the following notation:

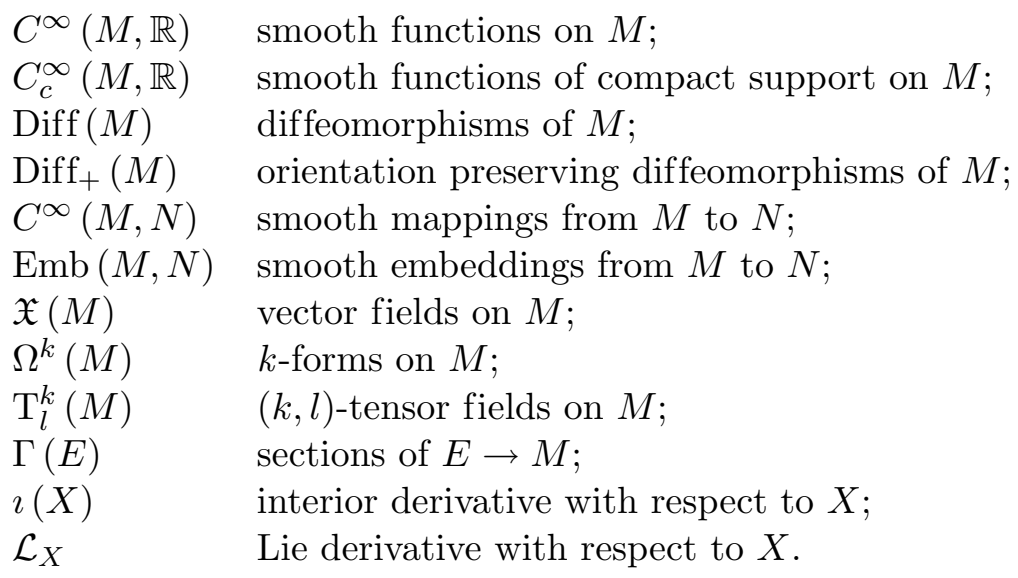

In the absence of summation signs repeated indices are summed over. 


\section{Basic definitions}

We begin by describing the "Convenient Setup" of Frölicher, Kriegl, and Michor in order to establish what we will mean by smoothness, tangent vectors, etc. on some infinite dimensional manifolds. Many definitions will be taken verbatim from [9]. All references like [9, X.X] in this section refer to sections in [9].

\subsection{Locally convex spaces}

A real topological vector space $E$ is a vector space equipped with a topology under which addition $+: E \times E \rightarrow E$ and scalar multiplication $\mathbb{R} \times E \rightarrow E$ are continuous.

A subset $C$ in $E$ is said to be

1) circled if $\lambda C \subset C$ whenever $|\lambda| \leq 1$;

2) convex if $\lambda_{1} C+\lambda_{2} C \subset C$ for all $\lambda_{1}, \lambda_{2} \geq 0$ satisfying $\lambda_{1}+\lambda_{2}=1$;

3) absolutely convex if $C$ is circled and convex.

A locally convex space is a Hausdorff topological vector space $E$, for which every neighbourhood of 0 contains an absolutely convex neighbourhood of 0 .

\subsection{Bounded sets}

A subset $C$ of a locally convex space $E$ is bounded if for each neighbourhood $U$ of 0 there exists a $\rho>0$ such that $C \subset \rho U$. The family of all bounded sets in $E$ is called the bornology of $E$. A linear map $T: E \rightarrow F$ between locally convex spaces is bounded if it maps bounded sets to bounded sets $[9,1.1]$.

\section{$2.3 \quad$ Smooth curves}

Let $E$ be a locally convex space. A curve $c: \mathbb{R} \rightarrow E$ is called differentiable if the derivative $c^{\prime}(t):=\lim _{h \rightarrow 0} \frac{1}{h}[c(t+h)-c(t)]$ at $t$ exists for all $t$. A curve $c: \mathbb{R} \rightarrow E$ is called smooth if all iterated derivatives exist. The set of all smooth curves in $E$ will be denoted by $C^{\infty}(\mathbb{R}, E)[9$, $1.2]$.

One would hope that reasonable definitions of smoothness would imply that "diffeomorphisms" are homeomorphisms. For this purpose we will make use of another topology on locally convex spaces.

\subsection{The $c^{\infty}$-topology}

The $c^{\infty}$-topology on a locally convex space $E$ is the finest topology for which all smooth curves $c: \mathbb{R} \rightarrow E$ are continuous $[9,2.12]$. The $c^{\infty}$-topology is finer than the locally convex topology on $E[9,4.7]$. If $E$ is a Fréchet space, (i.e. a complete and metrizable locally convex space), then the two topologies coincide $[9,4.1,4.11]$.

\subsection{Convenient vector spaces}

A convenient vector space is a locally convex space $E$ with the following property: For any $c_{1} \in C^{\infty}(\mathbb{R}, E)$ there is a $c_{2} \in C^{\infty}(\mathbb{R}, E)$ with $c_{2}^{\prime}=c_{1}$. Any $c^{\infty}$-closed subspace of a convenient vector space is convenient $[9,2.12,2.13,2.14]$. 


\subsection{Space of curves}

The set of smooth curves $C^{\infty}(\mathbb{R}, E)$ in a convenient vector space $E$ has a natural convenient structure. Moreover, a locally convex space $E$ is convenient if and only if $C^{\infty}(\mathbb{R}, E)$ is convenient $[9,3.7]$.

We would like to study sets that can be locally modeled on convenient vector spaces. To define "smooth transition functions" we need to define smooth mappings between convenient vector spaces.

\section{Convention}

For the rest of this section $E$ and $F$ will denote convenient vector spaces.

\subsection{Mappings between convenient vector spaces}

Let $U \subset E$ be a $c^{\infty}$-open subset. A mapping $f: U \rightarrow F$ is called smooth if it maps smooth curves in $U$ to smooth curves in $F$. Let $C^{\infty}(U, F)$ denote the set of all smooth mappings $f: U \rightarrow F$, equipped with the finest topology on $C^{\infty}(U, F)$ for which all maps $c^{*}: C^{\infty}(U, F) \rightarrow C^{\infty}(\mathbb{R}, F)$, given by pullback along smooth curves (i.e. $c^{*}(f)=f \circ c$ ), are continuous. Then $C^{\infty}(U, F)$ is a convenient vector space $[9,3.11]$.

\subsection{Spaces of linear mappings}

Let $L(E, F)$ denote the set of all bounded linear mappings from $E$ to $F$. Then $L(E, F)$ is contained in $C^{\infty}(E, F)[9,2.11]$ and inherits a convenient structure [9, 3.17]. The set of invertible maps in $L(E, F)$ with bounded inverse will be denoted by $G L(E, F)$.

\subsection{The differentiation operator and chain rule}

Let $U \subset E$ be a $c^{\infty}$-open subset. The differentiation operator

$$
\mathrm{d}: C^{\infty}(U, F) \rightarrow C^{\infty}(U, L(E, F)), \quad \mathrm{d} f(x) v:=\lim _{t \rightarrow 0} \frac{f(x+t v)-f(x)}{t}
$$

exists, is linear and bounded (smooth). Note that the above limit is taken in the locally convex topology of $F$. Also the chain rule

$$
\mathrm{d}(f \circ g)(x) v=\mathrm{d} f(g(x)) \mathrm{d} g(x) v
$$

holds $[9,3.18]$.

\subsection{Examples of convenient vector spaces}

The following spaces have natural convenient structures:

$$
\begin{array}{ll}
L_{\text {alt }}^{k}(E, F) & \text { bounded alternating multilinear maps } E \times \cdots \times E \rightarrow F[9,5.9,5.13] ; \\
C^{\infty}(M, \mathbb{R}) & \text { smooth functions on a finite dimensional manifold } M[9,6.1] ; \\
C_{c}^{\infty}(M, \mathbb{R}) & \text { smooth functions of compact support on a finite } \\
& \text { dimensional manifold } M[9,6.2] ; \\
\Gamma_{c}(Q) & \text { compactly supported smooth sections of a vector bundle } Q \rightarrow M ; \\
& \text { with finite dimensional total space, base, and fibers }[9,30.4] .
\end{array}
$$




\subsection{Manifolds}

A chart $(U, \varphi)$ on a set $M$ is a bijection $\varphi: U \rightarrow E_{U}$ from a subset $U \subset M$ onto a $c^{\infty}$-open set in a convenient vector space $E_{U}$. A family of charts $\left(U_{\alpha}, \varphi_{\alpha}\right)_{\alpha \in A}$ is called an atlas for $M$, if the $U_{\alpha}$ cover $M$ and all transition functions $\varphi_{\alpha \beta}:=\varphi_{\alpha} \circ \varphi_{\beta}^{-1}: \varphi_{\beta}\left(U_{\alpha} \cap U_{\beta}\right) \rightarrow \varphi_{\alpha}\left(U_{\alpha} \cap U_{\beta}\right)$ are smooth. Two atlases are equivalent if their union is again an atlas on $M$. A smooth manifold $M$ is a set together with an equivalence class of atlases on it $[9,27.1]$.

\subsection{Smooth mappings between manifolds}

A mapping $f: M \rightarrow N$ between manifolds is smooth if for every $x \in M$ and chart $(V, \psi)$ on $N$ with $f(x) \in V$ there exists a chart $(U, \varphi)$ on $M$ with $x \in U$ and $f(U) \subset V$ such that $\psi \circ f \circ \varphi^{-1}$ is smooth. So a mapping $f: M \rightarrow N$ is smooth if and only if it maps smooth curves to smooth curves. A smooth mapping $f: M \rightarrow N$ is a diffeomorphism if it is a bijection and if its inverse is smooth [9, 27.2]. The set of smooth maps from $M$ to $N$ will be denoted by $C^{\infty}(M, N)$.

\subsection{Submanifolds}

A subset $N$ of a smooth manifold $M$ is called a submanifold, if for each $x \in N$ there is a chart $(U, \varphi)$ of $M$ such that $\varphi(U \cap N)=\varphi(U) \cap F_{U}$, where $F_{U}$ is a $c^{\infty}$-closed linear subspace of the convenient model space $E_{U}[9,27.11]$. A curve in a submanifold $N$ of $M$ is smooth if and only if it is smooth as a curve in $M$.

\subsection{Tangent spaces of a convenient vector space}

Let $a \in E$. A tangent vector with base point $a$ is a pair $(a, X)$ with $X \in E$. For each neighbourhood $U$ of $a$ in $E$, a tangent vector $(a, X)$ defines a derivation $C^{\infty}(U, \mathbb{R}) \rightarrow \mathbb{R}$ by $X_{a} f:=\mathrm{d} f(a)(X)[9,28.1]$.

Remark 1. In [9] these tangent vectors are called kinematic tangent vectors since they can be realized as derivatives $c^{\prime}(0)$ at 0 of smooth curves $c: \mathbb{R} \rightarrow E$. This is to distinguish them from more general derivations which are called operational tangent vectors.

\subsection{The tangent bundle}

Let $M$ be a smooth manifold with an atlas $\left(U_{\alpha}, \varphi_{\alpha}\right)$ indexed by $\alpha \in A$. On the disjoint union

$$
\bigsqcup_{\alpha \in A} \mathcal{U}_{\alpha} \times E_{\alpha} \times\{\alpha\}
$$

define the following equivalence relation:

$$
(x, v, \alpha) \sim(y, w, \beta) \Longleftrightarrow x=y \text { and } \mathrm{d} \varphi_{\alpha \beta}\left(\varphi_{\beta}(y)\right) w=v
$$

A tangent vector at $x \in M$ is an equivalence class $[(x, v, \alpha)]$. The quotient $\bigsqcup_{\alpha \in A} \mathcal{U}_{\alpha} \times E_{\alpha} \times\{\alpha\} / \sim$ will be called the tangent bundle of $M$ and will be denoted by $T M$.

Let $\pi: T M \rightarrow M$ denote the projection $[(x, v, \alpha)] \mapsto x$. TM inherits a smooth manifold structure from $M$. For $x \in M$ the set $T_{x} M:=\pi^{-1}(x)$ is called the tangent space at $x$. Since each transition function $\varphi_{\alpha \beta}$ is smooth, each differential $\mathrm{d} \varphi_{\alpha \beta}(x)$ is bounded linear, which means each tangent space $T_{x} M$ has a well defined bornology independent of the choice of chart (cf. [9, $1.1,2.11])$.

Alternatively, we can describe tangent vectors to a smooth manifold by means of equivalence classes of smooth curves. We will say that two smooth curves $c_{1}$ and $c_{2}$ in $M$ are equivalent at 
$x \in M$, (and write $c_{1} \sim_{x} c_{2}$ ), if $c_{1}(0)=x=c_{2}(0)$ and $\left.\frac{d}{d t}\right|_{t=0} \varphi_{\alpha} \circ c_{1}(t)=\left.\frac{d}{d t}\right|_{t=0} \varphi_{\alpha} \circ c_{2}(t)$ for a chart $\varphi_{\alpha}$ in an atlas $\left(U_{\alpha}, \varphi_{\alpha}\right)_{\alpha \in A}$ on $M$. The tangent space at $x$ then is equal to $C^{\infty}(\mathbb{R}, M) / \sim_{x}$ (compare with [9, 28.12]).

\subsection{Tangent mappings}

Let $f: M \rightarrow N$ be a smooth mapping between manifolds. Then $f$ induces a linear map $\mathrm{d} f(x): T_{x} M \rightarrow T_{f(x)} N$ for each $x \in M$ by the following formula. If $X=[c] \in C^{\infty}(\mathbb{R}, M) / \sim_{x}$ then $\mathrm{d} f(x) X:=[f \circ c]$. This defines a fiberwise linear map $\mathrm{d} f: T M \rightarrow T N$ called the differential of $f$ (compare with $[9,28.15]$ ).

\subsection{Distributions}

A distribution on a smooth manifold $M$ is an assignment to each point $x \in M$ a $c^{\infty}$-closed subspace $\mathcal{D}_{x}$ of $T_{x} M$. If $\mathcal{D}=\left\{\mathcal{D}_{x}\right\}$ is a distribution on a manifold $M$ and $i: N \hookrightarrow M$ is the inclusion map of a connected submanifold $N$ of $M$, then $N$ is called an integral manifold of $\mathcal{D}$ if $\mathrm{d} i\left(T_{x} N\right)=\mathcal{D}_{i(x)}$ for all $x \in N$. An integral manifold of $\mathcal{D}$ is called maximal if it is not properly contained in any other integral manifold.

Let $\mathcal{D}$ be a distribution on a manifold $M$. The set of locally defined vector fields $X$ on $M$ satisfying $X(x) \in \mathcal{D}_{x}$ will be denoted by $\mathfrak{X}_{\mathcal{D}}(M)$.

Remark 2. In finite dimensions such distributions defined without any assumptions regarding continuity or smoothness are sometimes called "generalized distributions". If a generalized distribution $\mathcal{D}$ is "smooth" in the sense that every $v \in \mathcal{D}_{x} \subset T_{x} M$ can be realized as $X(x)$ for a locally defined vector field $X \in \mathfrak{X}_{\mathcal{D}}(M)$, then there exist results on integrability of such distributions (see e.g. [18, 19, 16, 17]).

\subsection{Foliations}

Let $M$ be a smooth manifold. A foliation of $M$ is a distribution $\mathcal{F}=\left\{\mathcal{F}_{x}\right\}$ on $M$, for which there exists an atlas $\left(U_{\alpha}, \varphi_{\alpha}\right)$ of charts $\varphi_{\alpha}: U_{\alpha} \rightarrow E_{\alpha}$ on $M$ and a family of $c^{\infty}$-closed subspaces $\left\{F_{\alpha} \subset E_{\alpha}\right\}$, such that the inverse image under $\varphi_{\alpha}$ of translations of $F_{\alpha}$ are integral manifolds of $\mathcal{F}$, and such that if $N \subset U_{\alpha}$ is an integral manifold of $\mathcal{F}$ then $\varphi_{\alpha}(N)$ is contained in a translation of $F_{\alpha}$. The charts $\varphi_{\alpha}$ will be called distinguished charts.

Let $\varphi_{\alpha}: U_{\alpha} \rightarrow E_{\alpha}$ be a distinguished chart of a foliation $\mathcal{F}$ of $M$ and $y+F_{\alpha}$ a translation of $F_{\alpha} \subset E_{\alpha}$. Then $\psi_{\alpha, y}:=\left.\varphi_{\alpha}\right|_{\varphi^{-1}\left(y+F_{\alpha}\right)}-y$ defines a chart into $F_{\alpha}$, and the set of all such charts $\psi_{\alpha, y}$ defines an alternative smooth structure on the set $M$ modeled on the spaces $F_{\alpha}$. The set $M$ equipped with this alternative manifold structure will be denoted by $M^{\mathcal{F}}$. A leaf of the foliation $\mathcal{F}$ is a connected component of $M^{\mathcal{F}}$. Since $T_{x} M^{\mathcal{F}}=\mathcal{F}_{x}$ for all $x \in M$, every leaf is a maximal integral manifold of $\mathcal{F}$ (compare with $[9,27.16]$ ).

Remark 3. Our definition of foliation differs from the definition in $[9,27.16]$ because we wish to describe foliations on manifolds modeled on different convenient spaces in different charts.

\subsection{Fiber bundles}

A fiber bundle $(Q, p, M)$ consists of manifolds $Q$ (the total space), $M$ (the base), and a smooth mapping $p: Q \rightarrow M$ (the projection) such that for every $x \in M$ there exists an open neighbourhood $U$ of $x$, a smooth manifold $S_{U}$, and a diffeomorphism $\psi$ such that the following diagram 
commutes:

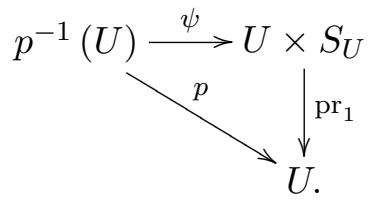

Such a pair $(U, \psi)$ as above is called a fiber bundle chart. A fiber bundle atlas $\left(U_{\alpha}, \psi_{\alpha}\right)_{\alpha \in A}$ is a set of fiber bundle charts such that $\left\{U_{\alpha}\right\}_{\alpha \in A}$ is an open cover of $M$. If we fix a fiber bundle atlas, then $\psi_{\alpha} \circ \psi_{\beta}^{-1}(x, s)=\left(x, \psi_{\alpha \beta}(x, s)\right)$ where $\psi_{\alpha \beta}:\left(U_{\alpha} \cap U_{\beta}\right) \times S_{\beta} \rightarrow S_{\alpha}$ is smooth, and where $\psi_{\alpha \beta}(x, \cdot)$ is a diffeomorphism of $S_{\beta}$ onto $S_{\alpha}$ for each $x \in U_{\alpha \beta}:=U_{\alpha} \cap U_{\beta}$. The mappings $\psi_{\alpha \beta}$ are called the transition functions of the bundle. When $S_{U_{\alpha}}=S$ for all charts $\left(U_{\alpha}, \psi_{\alpha}\right)$ for some smooth manifold $S$, then $S$ is called the standard fiber (compare with [9, 37.1]).

Remark 4. Our definition of a fiber bundle differs from the definition in $[9,37.1]$ in the sense that it allows for different $S_{U}$ for different neighbourhoods $U$.

\subsection{Vector bundles}

Let $(Q, p, M)$ be a fiber bundle. A fiber bundle chart $(U, \psi)$ is called a vector bundle chart if $S_{U}$ is a convenient vector space. Two vector bundle charts $\left(U_{\alpha}, \psi_{\alpha}\right)$ and $\left(U_{\beta}, \psi_{\beta}\right)$ are compatible if the transition function $\psi_{\alpha \beta}$ is bounded and linear in the fibers, i.e. $\psi_{\alpha \beta}(x, s)=\left(x, \phi_{\alpha \beta}(x) s\right)$ for some mapping $\phi_{\alpha \beta}: U_{\alpha \beta} \rightarrow G L\left(S_{\beta}, S_{\alpha}\right) \subset L\left(S_{\beta}, S_{\alpha}\right)$. A vector bundle atlas is a fiber bundle atlas $\left(U_{\alpha}, \psi_{\alpha}\right)_{\alpha \in A}$ consisting of pairwise compatible vector bundle charts. Two vector bundle atlases are equivalent if their union is again a vector bundle atlas. A vector bundle $(Q, p, M)$ is a fiber bundle together with an equivalence class of vector bundle atlases (compare with $[9,29.1])$.

Remark 5. Here again our definition differs from that in $[9,29.1]$ in that we allow for different $S_{U}$ in different neighbourhoods $U$. However, this more general version of vector bundles is subsequently used implicitly throughout the text (see e.g. [9, 29.9] and [9, 29.10] where the tangent bundle $T M$ of any smooth manifold $M$ is taken to be a vector bundle).

\subsection{Constructions with vector bundles}

If $Q \rightarrow M$ and $R \rightarrow M$ are vector bundles then we have vector bundles $Q^{*}, L(Q, R)$, and $L_{\text {alt }}^{k}(Q, R)$ whose fibers over $x \in M$ are $\left(Q_{x}\right)^{*}$ (the space of bounded linear functionals on $Q_{x}$ ), $L\left(Q_{x}, R_{x}\right)$ and $L_{\text {alt }}^{k}\left(Q_{x}, R_{x}\right)$ respectively [9, 29.5].

Remark 6. We will always use $E^{*}$ to denote the space of bounded linear functionals on a locally convex space $E$. In [9] $E^{*}$ is reserved for the space of continuous (in the locally convex topology) linear functionals while $E^{\prime}$ is used to denote the space of bounded linear functionals.

\subsection{Cotangent bundles}

Since $T M$ is a vector bundle for any manifold $M$, the bundle $(T M)^{*}$ with fiber over $x \in M$ equal to $\left(T_{x} M\right)^{*}$ is also a vector bundle. This vector bundle is called the cotangent bundle of $M$ and will be denoted by $T^{*} M[9,33.1]$.

\subsection{Spaces of sections of vector bundles}

A section of a vector bundle $p: Q \rightarrow M$ is a smooth map $s: M \rightarrow Q$ such that $p \circ s=\operatorname{Id}_{M}$. The set of sections of $p: Q \rightarrow M$ will be denoted by $\Gamma(Q)$, and the set of sections with compact 
support by $\Gamma_{c}(Q)$. The space $\Gamma(Q)$ has a natural convenient structure [9, 30.1]. If $M$ is finite dimensional and paracompact then $\Gamma_{c}(Q)$ has a natural convenient structure [9, 30.4], and if $W \subset Q$ is an open subset, then $\left\{s \in \Gamma_{c}(Q) \mid s(M) \subset W\right\}$ is $c^{\infty}$-open in $\Gamma_{c}(Q)$ [9, 30.10]. If $p: Q \rightarrow M$ is a finite dimensional vector bundle over a finite dimensional paracompact base, then the $c^{\infty}$-topology on $\Gamma_{c}(Q)$ is induced from the Whitney $C^{\infty}$-topology on $C^{\infty}(M, Q)$ (which coincides with the Fréchet topology; see Section 2.4) [9, 41.13].

We will be interested primarily in sets that can be locally modeled on spaces of sections of vector bundles. To understand notions of smoothness on such sets, it is enough to identify the smooth curves.

\subsection{Curves in spaces of sections}

Let $Q \rightarrow M$ be a vector bundle. A curve $c: \mathbb{R} \rightarrow \Gamma(Q)$ is smooth if and only if the associated map $c^{\wedge}: \mathbb{R} \times M \rightarrow Q$ defined by $c^{\wedge}(t, x):=c(t)(x)$ is smooth $[9,30.8]$.

\subsection{Example: manifold of mappings}

Let $M$ and $N$ be finite dimensional manifolds. The space $C^{\infty}(M, N)$ is a smooth manifold modeled on spaces $\Gamma_{c}\left(f^{*} T N\right)$ of compactly supported sections of the pullback bundle along $f \in C^{\infty}(M, N)[9,42.1]$. The charts can be described as follows. Choose a Riemannian metric on $N$ and let $\exp : T N \supset U \rightarrow N$ be the smooth exponential map of this metric. If $\pi_{N}: T N \rightarrow N$ denotes the projection of the tangent bundle, then we can assume that $\left(\pi_{N}, \exp \right): U \rightarrow N \times N$ is a diffeomorphism onto an open neighbourhood $W$ of the diagonal. For $f, g \in C^{\infty}(M, N)$, we will write $f \sim g$ if $f$ and $g$ differ only on a compact set in $M$. The charts $\left(V_{f}, \psi_{f}\right)$ indexed by $f \in C^{\infty}(M, N)$ are given by

$$
\begin{aligned}
& V_{f}:=\left\{g \in C^{\infty}(M, N) \mid g \sim f,(f(x), g(x)) \in W \text { for all } x \in M\right\}, \\
& \psi_{f}: V_{f} \rightarrow \Gamma_{c}\left(f^{*} T N\right), \\
& \psi_{f}(g)(x):=\left(x, \exp _{f(x)}^{-1}(g(x))\right)=\left(x,\left(\left(\pi_{N}, \exp \right)^{-1}(f(x), g(x))\right)\right) .
\end{aligned}
$$

\subsection{Vector fields}

Let $M$ be a smooth manifold. A vector field $X$ on $M$ is a smooth section of the tangent bundle $T M[9,32.1]$. The set of all vector fields on $M$ will be denoted by $\mathfrak{X}(M)$. Each vector field $X$ specifies a map

$$
\begin{aligned}
& C^{\infty}(M, \mathbb{R}) \rightarrow C^{\infty}(M, \mathbb{R}), \\
& f \mapsto X f, \\
& X f(x):=\mathrm{d} f(x) X(x) .
\end{aligned}
$$

\subsection{The Lie bracket}

Let $X$ and $Y$ be smooth vector fields on a manifold $M$. Each such vector field is a smooth mapping $M \rightarrow T M$ between manifolds, and so it makes sense to compute the differentials $\mathrm{d} X$ and $\mathrm{d} Y$. The Lie bracket $[X, Y]$ of $X$ and $Y$ is the vector field on $M$ given by the expression

$$
[X, Y]=\mathrm{d} Y(X)-\mathrm{d} X(Y) .
$$

The bracket $[\cdot, \cdot]: \mathfrak{X}(M) \times \mathfrak{X}(M) \rightarrow \mathfrak{X}(M)$ defines a Lie algebra structure on $\mathfrak{X}(M)[9,32.5$, 32.8 . 


\subsection{Differential forms}

A differential $k$-form on a manifold $M$ is a section $\omega \in \Gamma\left(L_{\text {alt }}^{k}(T M, M \times \mathbb{R})\right)$. The set of all differential $k$-forms will be denoted by $\Omega^{k}(M)$ [9, 33.22].

\subsection{The pullback of a differential form}

Let $f: N \rightarrow M$ be a smooth mapping and $\omega \in \Omega^{k}(M)$ be a differential $k$-form on $M$. The pullback $f^{*} \omega \in \Omega^{k}(N)$ of $\omega$ is defined by

$$
f^{*} \omega_{x}\left(X_{1}, \ldots, X_{k}\right):=\omega_{f(x)}\left(\mathrm{d} f(x) X_{1}, \ldots, \mathrm{d} f(x) X_{k}\right),
$$

see $[9,33.9]$.

\subsection{The insertion operator}

For a vector field $X \in \mathfrak{X}(M)$ on a manifold $M$, the insertion operator $\imath(X)$ is defined by

$$
\begin{aligned}
\imath(X): & \Gamma\left(L_{\mathrm{alt}}^{k}(T M, M \times \mathbb{R})\right) \rightarrow \Gamma\left(L_{\mathrm{alt}}^{k-1}(T M, M \times \mathbb{R})\right) \\
& (\iota(X) \omega)\left(Y_{1}, \ldots, Y_{k-1}\right):=\omega\left(X, Y_{1}, \ldots, Y_{k-1}\right),
\end{aligned}
$$

see $[9,33.10]$.

\subsection{The exterior derivative}

Let $U \subset E$ be a $c^{\infty}$-open subset and let $\omega \in C^{\infty}\left(U, L_{\text {alt }}^{k}(E, \mathbb{R})\right)$ be a differential $k$-form on $U$. The exterior derivative $d \omega \in C^{\infty}\left(U, L_{\text {alt }}^{k+1}(E, \mathbb{R})\right)$ of $\omega$ is the skew symmetrization of the differential d $\omega$ :

$$
(d \omega)(x)\left(X_{0}, \ldots, X_{k}\right)=\sum_{i=0}^{k}(-1)^{i} \mathrm{~d} \omega(x)\left(X_{i}\right)\left(X_{0}, \ldots, \hat{X}_{i}, \ldots, X_{k}\right) .
$$

(Note that the differential $\mathrm{d} \omega$ with plain text $\mathrm{d}$ is used to define the exterior derivative d $\omega$ with italicized $d$.) If $\omega$ is a differential $k$-form on a manifold $M$, then this local formula defines a differential $k+1$-form $d \omega$ on $M$. The above local expression for the exterior derivative induces the global formula

$$
\begin{aligned}
(d \omega)(x)\left(X_{0}, \ldots, X_{k}\right)= & \sum_{i=0}^{k}(-1)^{i} X_{i}\left(\omega \circ\left(X_{0}, \ldots, \hat{X}_{i}, \ldots, X_{k}\right)\right) \\
& +\sum_{i<j}(-1)^{i+j} \omega \circ\left(\left[X_{i}, X_{j}\right], X_{0}, \ldots, \widehat{X}_{i}, \ldots, \widehat{X}_{j}, \ldots, X_{k}\right),
\end{aligned}
$$

where $X_{0}, \ldots, X_{k} \in \mathfrak{X}(M)[9,33.12]$.

\subsection{Lie groups}

A Lie group $G$ is a smooth manifold and a group such that multiplication $\mu: G \times G \rightarrow G$ and inversion $\nu: G \rightarrow G$ are smooth. The Lie algebra of a Lie group $G$ is the tangent space to $G$ at the identity $e$, which inherits a Lie bracket from the identification with left invariant vector fields. The Lie algebra will be denoted either by $\mathfrak{g}$ or $\operatorname{Lie}(G)[9,36.1,36.3]$. 


\subsection{Basic differential forms}

Let $l: G \times M \rightarrow M$ be a smooth action of a Lie group $G$ on a smooth manifold $M$. Let $l_{g}: M \rightarrow M$ denote the left multiplication mapping $x \mapsto g \cdot x$. For $\xi \in \mathfrak{g}$ the generating vector field $\xi_{M}$ is defined by $\xi_{M}(x):=\mathrm{d} l_{(e, x)}(\xi, 0)$. A differential $k$-form $\omega \in \Omega^{k}(M)$ on $M$ is $G$ invariant if $l_{g}^{*} \omega=\omega$ for all $g \in G$ and horizontal if $\omega\left(\xi_{M}, \cdot\right)=0 \in \Omega^{k-1}(M)$ for all $\xi \in \mathfrak{g}$. A differential $k$-form $\omega \in \Omega^{k}(M)$ is basic if it is both $G$-invariant and horizontal. The set of all basic $k$-forms on $M$ will be denoted by $\Omega_{\text {hor }}^{k}(M)^{G}[9,37.23]$.

\subsection{Principal $G$ bundles}

Let $G$ be a Lie group. A principal $G$ bundle is a fiber bundle $(P, p, M, G)$ with standard fiber $G$ whose transition functions act on $G$ via left translation: There is a family of smooth mappings $\left\{\phi_{\alpha \beta}: U_{\alpha \beta} \rightarrow G\right\}$ that satisfy the cocycle condition $\phi_{\alpha \beta}(x) \phi_{\beta \gamma}(x)=\phi_{\alpha \gamma}(x)$ for $x \in U_{\alpha} \cap U_{\beta} \cap U_{\gamma}$, $\phi_{\alpha \alpha}(x)=e($ the identity in $G)$, and $\psi_{\alpha \beta}(x, g)=\phi_{\alpha \beta}(x) \cdot g[9,37.7,37.8]$. The pull back through the projection $p^{*}: \Omega^{k}(M) \rightarrow \Omega_{\mathrm{hor}}^{k}(P)^{G}$ is an isomorphism [9, 37.30].

\subsection{Diffeomorphism groups}

The following diffeomorphism groups are examples of infinite dimensional Lie groups:

- the group $\operatorname{Diff}(M)$ of diffeomorphisms of a finite dimensional paracompact manifold $M$; the Lie algebra is the space $\mathfrak{X}_{c}(M)$ of compactly supported vector fields on $M$. In fact, $\operatorname{Diff}(M)$ is open in $C^{\infty}(M, M)$ so the tangent space at each $f \in \operatorname{Diff}(M)$ is equal to $\Gamma\left(f^{*} T M\right)[9,43.1]$.

- the group $\operatorname{Symp}(M)$ of symplectomorphisms of a (finite dimensional) symplectic manifold $(M, \sigma)$; the Lie algebra is the space $\mathfrak{X}_{c}^{\text {symp }}(M)$ of compactly supported symplectic vector fields [9, 43.12]. $\left(\phi \in \operatorname{Diff}(M)\right.$ is a symplectomorphism if $\phi^{*} \sigma=\sigma ; X \in \mathfrak{X}(M)$ is a symplectic vector field if $\mathcal{L}_{X} \sigma=0$.)

- the group $\operatorname{Ham}(M)$ of Hamiltonian symplectomorphisms of a (finite dimensional) symplectic manifold $(M, \sigma)$; the Lie algebra is the space $\mathfrak{X}_{c}^{\text {ham }}(M)$ of compactly supported Hamiltonian vector fields $[9,43.12,43.13] . \quad(X \in \mathfrak{X}(M)$ is a Hamiltonian vector field if $\imath(X) \sigma$ is exact; $\phi \in \operatorname{Symp}(M)$ is a Hamiltonian symplectomorphism if it is the time 1 flow of a time dependent Hamiltonian vector field.)

Remark 7. In heuristic approaches to infinite dimensional Lie groups, the Lie algebra to the group Diff $(M)$ of diffeomorphisms of a finite dimensional manifold $M$ is often taken to be the space of smooth vector fields on $M$. In the convenient setup, the Lie algebra of $\operatorname{Diff}(M)$ is given by the space $\mathfrak{X}_{c}(M)$ of compactly supported vector fields on $M$ because of the choice of charts.

\subsection{The adjoint representation}

Let $G L(E)$ denote the set of bounded invertible linear transformations of $E$. Let $G$ be a Lie group with Lie algebra $\mathfrak{g}$. Every element $g \in G$ defines an automorphism $\psi_{g}: G \rightarrow G$ by conjugation: $\psi_{g}(a):=g a g^{-1}$. The adjoint representation of $G$ denoted by $\mathrm{Ad}: G \rightarrow G L(\mathfrak{g}) \subset$ $L(\mathfrak{g}, \mathfrak{g})$ is given by $\operatorname{Ad}(g):=\mathrm{d}_{e} \psi_{g}: \mathfrak{g} \rightarrow \mathfrak{g}$ for $g \in G$. The adjoint representation of $\mathfrak{g}$ denoted by ad $: \mathfrak{g} \rightarrow \mathfrak{g l}(\mathfrak{g}):=L(\mathfrak{g}, \mathfrak{g})$ is given by ad $:=\mathrm{d}_{e} \operatorname{Ad}[9,36.10]$.

\subsection{Weak symplectic manifolds}

A 2-form $\sigma \in \Omega^{2}(M)$ on a manifold $M$ is called a weak symplectic structure on $M$ if it is closed $(d \sigma=0)$ and if its associated vector bundle homomorphism $\sigma^{b}: T M \rightarrow T^{*} M$ is injective. This 
last condition is equivalent to weak nondegeneracy: for every $x \in M$ and $v \in T_{x} M$ there exists a $w \in T_{x} M$ such that $\sigma_{x}(v, w) \neq 0$. If $\sigma^{b}: T M \rightarrow T^{*} M$ is invertible with a smooth inverse then $\sigma$ is called a strong symplectic structure on $M[9,48.2]$. A vector field $X \in \mathfrak{X}(M)$ will be called Hamiltonian if $\iota(X) \sigma=d H$ for some $H \in C^{\infty}(M, \mathbb{R})$, and the function $H$ will called a Hamiltonian of $X$.

\section{Isodrastic foliations}

In this section we will describe our approach towards describing Lagrangian submanifolds as Lagrangian embeddings modulo reparametrizations. We will show that the space of Lagrangian embeddings into a fixed symplectic manifold $(M, \omega)$ is a smooth manifold which has a natural foliation $\mathcal{E}$. Moreover, the space of Lagrangian embeddings of the form $L_{0} \hookrightarrow M$ is the total space of a principal $\operatorname{Diff}_{+}\left(L_{0}\right)$ bundle over the space of Lagrangian submanifolds in $M$. The leaves of $\mathcal{E}$ will turn out to be orbits of the group of Hamiltonian symplectomorphisms under the natural left composition action. Meanwhile the foliation $\mathcal{E}$ descends to a foliation $\mathcal{F}$ of the space of Lagrangian submanifolds in $M$. In all of these constructions, the key will be to use Weinstein's Lagrangian Neighbourhood Theorem which says that any symplectic manifold near a Lagrangian $L$ looks like a neighbourhood of the zero section in the cotangent bundle $T^{*} L$.

Let $(M, \omega)$ be a finite dimensional symplectic manifold. Let $L_{0}$ be an oriented, compact manifold of half the dimension of $M$.

\section{Notation}

By $\operatorname{Lag}\left(L_{0}, M\right)$ we will denote the set of Lagrangian embeddings of $L_{0}$ into $(M, \omega)$. That is,

$$
\operatorname{Lag}\left(L_{0}, M\right):=\left\{i \in \operatorname{Emb}\left(L_{0}, M\right) \mid i^{*} \omega=0\right\} .
$$

Let $Z^{k}(N)$ and $B^{k}(N)$ denote the set of closed and exact $k$-forms respectively on a manifold $N$. That is,

$$
Z^{k}(N):=\operatorname{Ker}(d) \cap \Omega^{k}(N), \quad B^{k}(N):=\operatorname{Im}(d) \cap \Omega^{k}(N) .
$$

We will show that $\operatorname{Lag}\left(L_{0}, M\right)$ is a smooth manifold by defining an atlas of charts using the following Lagrangian neighbourhood theorem of Weinstein:

Theorem 1 (see Theorem 6.1 and Corollary 6.2 in [21]). Let $L$ be a Lagrangian submanifold of a symplectic manifold $(M, \omega)$. Then there exists an open neighbourhood $U$ of $L$ and a symplectic embedding $\psi: U \rightarrow T^{*} L$ such that $\left.\psi\right|_{L}=1_{L}$ and $\psi^{*} \omega_{T^{*} L}=\omega$.

Proposition 1. $\operatorname{Lag}\left(L_{0}, M\right)$ is a smooth manifold modeled on the space $Z^{1}\left(L_{0}\right) \oplus \mathfrak{X}\left(L_{0}\right)$.

Proof. The idea of the proof is as follows. By Theorem 1, Lagrangian submanifolds near a given Lagrangian submanifold can be identified with the graphs of closed 1-forms in $T^{*} L_{0}$. It follows that Lagrangian embeddings near a given one can be identified with closed 1-forms viewed as maps from $L_{0}$ to $T^{*} L_{0}$ precomposed with diffeomorphisms of $L_{0}$.

Given $i \in \operatorname{Lag}\left(L_{0}, M\right)$, by Theorem 1 the embedding $i$ can be extended on a neighbourhood $W_{i}$ of the zero section in $T^{*} L_{0}$ to a symplectic embedding $\lambda_{i}: W_{i} \rightarrow M$. Let $V_{e}$ be a chart neighbourhood of the identity map $e \in \operatorname{Diff}\left(L_{0}\right)$ and denote by $\psi_{e}: V_{e} \rightarrow \mathfrak{X}\left(L_{0}\right)$ the corresponding chart as part of an atlas on Diff $\left(L_{0}\right)$. Define

$$
\begin{aligned}
& U_{i}:=\left\{l \in \operatorname{Lag}\left(L_{0}, M\right) \mid l=\lambda_{i} \circ \alpha \circ a, \alpha \in Z^{1}\left(L_{0}\right), \alpha\left(L_{0}\right) \subset W_{i}, a \in V_{e}\right\}, \\
& \varphi_{i}: U_{i} \rightarrow Z^{1}\left(L_{0}\right) \oplus \mathfrak{X}\left(L_{0}\right), \quad \varphi_{i}(l):=\left(\alpha, \psi_{e}(a)\right) .
\end{aligned}
$$


The space $\mathfrak{X}\left(L_{0}\right)=\Gamma\left(T L_{0}\right)$ is convenient by Section 2.10. The space $Z^{1}\left(L_{0}\right)$ is a $c^{\infty}$-closed subspace of $\Gamma\left(T^{*} L_{0}\right)$ since it is the kernel of the continuous map $d: \Gamma\left(T^{*} L_{0}\right) \rightarrow \Gamma\left(\bigwedge^{2} T^{*} L_{0}\right)$, and therefore it is convenient. The set $\left\{\alpha \in Z^{1}\left(L_{0}\right) \mid \alpha\left(L_{0}\right) \subset W_{i}\right\}$ is $c^{\infty}$-open in $Z^{1}\left(L_{0}\right)$ (see Section 2.23). Thus $\varphi_{i}$ is a bijection of $U_{i}$ onto a $c^{\infty}$-open subset of $Z^{1}\left(L_{0}\right) \oplus \mathfrak{X}\left(L_{0}\right)$.

The collection $\left(U_{i}, \varphi_{i}\right)_{i \in \operatorname{Lag}\left(L_{0}, M\right)}$ defines a smooth atlas on $\operatorname{Lag}\left(L_{0}, M\right)$, since the chart changings $\varphi_{i k}$ are smooth by smoothness of the exponential map, by smoothness of each symplectic embedding $\lambda_{i}$, and by Section 2.24.

To explicitly describe the tangent space to $\operatorname{Lag}\left(L_{0}, M\right)$ at a point $i$, we will make use of the following notation.

\section{Notation}

If $S_{0}$ is a manifold (not necessarily of half the dimension of $M$ ), then for every $i \in \operatorname{Emb}\left(S_{0}, M\right)$ we can view the tangent bundle $T S_{0}$ as a subbundle of the pullback bundle $i^{*} T M$. The symplectic form $\omega$ defines a vector bundle isomorphism $\omega^{b}: T M \rightarrow T^{*} M$, which induces a vector space isomorphism $\mu: \Gamma\left(i^{*} T M\right) \rightarrow \Gamma\left(i^{*} T^{*} M\right)$. There is a natural surjection from the pullback bundle $i^{*} T^{*} M$ onto the cotangent bundle $T^{*} S_{0}$. This induces a linear map $\nu: \Gamma\left(i^{*} T^{*} M\right) \rightarrow$ $\Gamma\left(T^{*} S_{0}\right)=\Omega^{1}\left(S_{0}\right)$. For $X \in \Gamma\left(i^{*} T M\right)$, let $\alpha_{X} \in \Omega^{1}\left(S_{0}\right)$ denote the image of $X$ under the composition $\nu \circ \mu$. That is,

$$
\begin{aligned}
& X \mapsto \alpha_{X} \in \Omega^{1}\left(S_{0}\right), \\
& \alpha_{X}(\xi):=\omega_{i(x)}(X(x), \mathrm{d} i(x) \cdot \xi) \quad \forall \xi \in T_{x} S_{0} .
\end{aligned}
$$

Also, set

$$
\begin{aligned}
& \left.\Gamma_{\text {closed }} i^{*} T M\right):=\left\{X \in \Gamma\left(i^{*} T M\right) \mid \alpha_{X} \in Z^{1}\left(S_{0}\right)\right\}, \\
& \Gamma_{\text {exact }}\left(i^{*} T M\right):=\left\{X \in \Gamma\left(i^{*} T M\right) \mid \alpha_{X} \in B^{1}\left(S_{0}\right)\right\} .
\end{aligned}
$$

Remark 8. If $Y \in \mathfrak{X}\left(L_{0}\right)$ then $\alpha_{Y}=0$ for all $i \in \operatorname{Lag}\left(L_{0}, M\right)$ since such embeddings are Lagrangian.

Proposition 2. For each $i \in \operatorname{Lag}\left(L_{0}, M\right)$, the sequence

$$
0 \rightarrow \mathfrak{X}\left(L_{0}\right) \stackrel{f_{1}}{\longrightarrow} \Gamma_{\text {closed }}\left(i^{*} T M\right) \stackrel{f_{2}}{\longrightarrow} Z^{1}\left(L_{0}\right) \rightarrow 0,
$$

where $f_{1}(Y)=\mathrm{d} i(Y)$ and $f_{2}(X)=\alpha_{X}$, is a $\operatorname{Diff}\left(L_{0}\right)$-equivariant exact sequence.

Proof. If $i \in \operatorname{Lag}\left(L_{0}, M\right)$ and $X \in \Gamma\left(i^{*} T M\right)$, then $\alpha_{X}=0$ if and only if $X$ is tangent to $i\left(L_{0}\right)$ since $i$ is Lagrangian. Thus $\operatorname{Ker}\left(f_{2}\right)=\operatorname{Im}\left(f_{1}\right)$. To check that $f_{2}$ is onto, let $\alpha \in Z^{1}\left(L_{0}\right)$. By Theorem 1, it is enough to prove the assertion when $M=T^{*} L_{0}$ and $i$ is the zero section inclusion. Let $\pi: T^{*} L_{0} \rightarrow L_{0}$ denote the canonical projection. Define $Z_{\alpha} \in \mathfrak{X}\left(T^{*} L_{0}\right)$ by

$$
\imath\left(Z_{\alpha}\right) \omega:=\pi^{*} \alpha .
$$

Then $\alpha_{Z_{\alpha} \circ i}=\alpha$, which means $f_{2}$ is surjective and so the sequence is exact.

Remark 9. Each symplectic embedding $\lambda_{i}: T^{*} L_{0} \supset W_{i} \rightarrow M$ defines a splitting map $s_{i}$ : $Z^{1}\left(L_{0}\right) \rightarrow \Gamma_{\text {closed }}\left(i^{*} T M\right)$ of the exact sequence (1) given by

$$
s_{i}(\alpha):=\left.\mathrm{d} \lambda_{i} \circ Z_{\alpha}\right|_{L_{0}}
$$

where $\imath\left(Z_{\alpha}\right) \omega_{T^{*} L_{0}}=\pi^{*} \alpha$. Under this splitting $\Gamma_{\text {closed }}\left(i^{*} T M\right)=Z^{1}\left(L_{0}\right) \oplus \mathfrak{X}\left(L_{0}\right)$. 
Proposition 3. For each $i \in \operatorname{Lag}\left(L_{0}, M\right)$,

$$
T_{i} \operatorname{Lag}\left(L_{0}, M\right)=\Gamma_{\text {closed }}\left(i^{*} T M\right) .
$$

Proof. Let $j_{t}$ be a smooth curve in $\operatorname{Lag}\left(L_{0}, M\right)$ such that $j_{0}=i$. For each $x \in L_{0}$ we have that $\left.\frac{d}{d t}\right|_{t=0} j_{t}(x) \in T_{i(x)} M$, which means $\left.\frac{d}{d t}\right|_{t=0} j_{t} \in \Gamma\left(i^{*} T M\right)$. The fact that $j_{t}^{*} \omega=0$ implies that $\left.\frac{d}{d t}\right|_{t=0} j_{t} \in \Gamma_{\text {closed }}\left(i^{*} T M\right)$. If $\left(U_{l}, \varphi_{l}\right)$ is a chart corresponding to a symplectic embedding $\lambda_{l}: T^{*} L_{0} \supset W_{l} \rightarrow M$ with $i \in U_{l}$, then the derivative $\left.\frac{d}{d t}\right|_{t=0} \varphi_{l}\left(j_{t}\right)$ in $Z^{1}\left(L_{0}\right) \oplus \mathfrak{X}\left(L_{0}\right) \subset$ $\left.T\left(T^{*} L_{0}\right)\right|_{L_{0}}$ is canonically identifed with $\left.\frac{d}{d t}\right|_{t=0} j_{t}$ via

$$
\left.\frac{d}{d t}\right|_{t=0} \varphi_{l}\left(j_{t}\right)=\left.\alpha_{\frac{d}{d t}}\right|_{t=0} j_{t}+\left.\mathrm{d} \pi \circ \mathrm{d} \lambda_{l}^{-1} \circ \frac{d}{d t}\right|_{t=0} j_{t}
$$

So, $T_{i} \operatorname{Lag}\left(L_{0}, M\right) \subset \Gamma_{\text {closed }}\left(i^{*} T M\right)$.

Conversely, suppose that $X \in \Gamma_{\text {closed }}\left(i^{*} T M\right)$ and denote by $\lambda_{i}: T^{*} L_{0} \supset W_{i} \rightarrow M$ the symplectic embedding associated to the chart $\left(U_{i}, \varphi_{i}\right)$. Define a smooth curve in $\operatorname{Symp}\left(T^{*} L_{0}\right)$ by $\psi_{t}(x, p):=\left(x, p+t \alpha_{X}(x)\right)$ and a vector field on $L_{0}$ by $Y:=\mathrm{d} \pi \circ \mathrm{d} \lambda_{i}^{-1} \circ X$. If $a_{t}$ denotes the flow generated by $Y$ on $L_{0}$ and $\mathbf{O}$ denotes the zero section, then $\left.\mathrm{d} \lambda_{i} \circ \frac{d}{d t}\right|_{t=0}\left(\psi_{t} \circ \mathbf{O} \circ a_{t}\right)=X$. Thus $X \in \Gamma_{\text {closed }}\left(i^{*} T M\right)$ corresponds to the class $\left[\lambda_{i} \circ \psi_{t} \circ \mathbf{O} \circ a_{t}\right]$ in $T_{i} \operatorname{Lag}\left(L_{0}, M\right)$.

The set Ham $(M)$ of Hamiltonian symplectomorphisms is a subgroup of $\operatorname{Symp}(M)$ (see e.g. [12]). So left composition defines an action of $\operatorname{Ham}(M)$ on $\operatorname{Lag}\left(L_{0}, M\right)$ via

$$
\phi \cdot i:=\phi \circ i \text {. }
$$

Proposition 4. The spaces $\Gamma_{\text {exact }}\left(i^{*} T M\right) \subset \Gamma_{\text {closed }}\left(i^{*} T M\right)$ and charts $\left(U_{i}, \varphi_{i}\right)$ for $i \in \operatorname{Lag}\left(L_{0}, M\right)$ define a foliation $\mathcal{E}$ of $\operatorname{Lag}\left(L_{0}, M\right)$, whose leaves consist of $\operatorname{Ham}(M)$ orbits.

Proof. Set $\mathcal{E}_{i}:=\Gamma_{\text {exact }}\left(i^{*} T M\right)$ for $i \in \operatorname{Lag}\left(L_{0}, M\right)$. We will first show that $\Gamma_{\text {exact }}\left(i^{*} T M\right)$ is a $c^{\infty}$-closed (i.e. convenient) subspace of $\Gamma_{\text {closed }}\left(i^{*} T M\right)$ for all $i \in \operatorname{Lag}\left(L_{0}, M\right)$. If $\Gamma_{\text {closed }}\left(i^{*} T M\right)$ is identified with $Z^{1}\left(L_{0}\right) \oplus \mathfrak{X}\left(L_{0}\right)$ via the splitting map $s_{i}: Z^{1}\left(L_{0}\right) \rightarrow \Gamma_{\text {closed }}\left(i^{*} T M\right)$, then to show that $\Gamma_{\text {exact }}\left(i^{*} T M\right)$ is $c^{\infty}$-closed in $\Gamma_{\text {closed }}\left(i^{*} T M\right)$ it is enough to show that $F:=B^{1}\left(L_{0}\right) \oplus$ $\mathfrak{X}\left(L_{0}\right)$ is $c^{\infty}$-closed in $Z^{1}\left(L_{0}\right) \oplus \mathfrak{X}\left(L_{0}\right)$. Let $c_{1}: \mathbb{R} \rightarrow F$ be a smooth curve in $F$. If $c_{1}(t)=$ $\left(d f_{t}, Y_{t}\right)$ then $p_{1}: t \mapsto f_{t}$ can be chosen to be a smooth curve in $C^{\infty}\left(L_{0}, \mathbb{R}\right)$. Since $C^{\infty}\left(L_{0}, \mathbb{R}\right)$ is convenient, there exists a smooth curve $p_{2}: t \mapsto g_{t}$ in $C^{\infty}\left(L_{0}, \mathbb{R}\right)$ such that $p_{2}^{\prime}=p_{1}$. Similarly for the curve $q_{1}: t \mapsto Y_{t}$ there exists a smooth curve $q_{2}: t \mapsto Z_{t}$ in $\mathfrak{X}\left(L_{0}\right)$ such that $q_{2}^{\prime}=q_{1}$. Then $c_{2}(t):=\left(d g_{t}, Z_{t}\right)$ is an antiderivative of $c_{1}$, i.e. $c_{2}^{\prime}=c_{1}$. This means $F$ is a convenient subspace.

We will next show that the $\operatorname{Ham}(M)$ orbits in $\operatorname{Lag}\left(L_{0}, M\right)$ are maximal integral manifolds of $\mathcal{E}=\left\{\mathcal{E}_{i}\right\}$. The tangent vectors to a $\operatorname{Ham}(M)$ orbit at a point $i \in \operatorname{Lag}\left(L_{0}, M\right)$ are of the form $X_{H} \circ i$ where $X_{H}$ is a Hamiltonian vector field on $M$. Since $\alpha_{X_{H} \circ i}=i^{*} d H$ it follows that $T_{i}(\operatorname{Ham}(M) \cdot i) \subset \mathcal{E}_{i}$. Conversely, if $X \in \mathcal{E}_{i}$ then there exists a Hamiltonian vector field $X_{H}$ defined on a neighbourhood of $i\left(L_{0}\right)$ satisfying $X=X_{H} \circ i$. By multiplying $H$ by a cutoff function which is equal to 1 near $i\left(L_{0}\right)$ we may assume that $X_{H}$ is defined on all of $M$. It follows that $\mathcal{E}_{i} \subset T_{i}(\operatorname{Ham}(M) \cdot i)$. So $\operatorname{Ham}(M)$ orbits are integral manifolds. To show they are maximal, we first consider the case when $M=T^{*} L_{0}$. Let $i: L_{0} \hookrightarrow T^{*} L_{0}$ denote the zero section inclusion and $\left(U_{i}, \varphi_{i}\right)$ the corresponding chart on $\operatorname{Lag}\left(L_{0}, T^{*} L_{0}\right)$. Let $j_{t}$ be a smooth curve in an integral manifold $N$ contained in $U_{i}$. For every $t, \frac{d}{d t} j_{t} \in \Gamma_{\text {exact }}\left(j_{t}^{*} T\left(T^{*} L_{0}\right)\right)$ which means $\alpha_{\frac{d}{d t} j_{t}}=d h_{t}$ for a family of functions $h_{t} \in C^{\infty}\left(L_{0}, \mathbb{R}\right)$. This family $h_{t}$ can be chosen to be a smooth curve in $C^{\infty}\left(L_{0}, \mathbb{R}\right)$. Since $j_{t}$ is contained in $U_{i}$, there exist smooth curves $\beta_{t}$ in $Z^{1}\left(L_{0}\right)$ and $a_{t}$ in $\operatorname{Diff}\left(L_{0}\right)$ such that $j_{t}=\beta_{t} \circ a_{t}$. Since $\frac{d}{d t}\left(\beta_{t} \circ a_{t}\right)=\left(\frac{d}{d t} \beta_{t}\right) \circ a_{t}+\mathrm{d} \beta_{t}\left(\frac{d}{d t} a_{t}\right)$, it follows that 
$-a_{t}^{*} \frac{d}{d t} \beta_{t}=d h_{t}$ for all $t$. Thus, $\beta_{t}=\psi_{t} \circ \beta_{0}$ where $\psi_{t}(x, p)=\left(x, p-\left(d \int_{0}^{t} h_{s} \circ a_{s}^{-1} d s\right)_{x}\right)$, which means $\beta_{t}$ is contained in the $\operatorname{Ham}\left(T^{*} L_{0}\right)$ orbit through $\beta_{0}$. For each $t$ we can write $\beta_{0} \circ a_{t}=b_{t} \circ \beta_{0}$ where $b_{t} \in \operatorname{Diff}\left(\operatorname{Graph}\left(\beta_{0}\right)\right)$ in such a way that $b_{t}$ is a smooth curve in $\operatorname{Diff}\left(\operatorname{Graph}\left(\beta_{0}\right)\right)$. Define $c_{t}:=b_{t} \circ b_{0}^{-1}$. By means of Theorem 1 we can identify an open neighbourhood of Graph $\left(\beta_{0}\right)$ with an open neighbourhood of the zero section in $T^{*} \operatorname{Graph}\left(\beta_{0}\right)$. Under this identification, if $C_{t}$ denotes the cotangent lift of $c_{t}$, i.e. $C_{t}(y, z)=\left(c_{t}(y),\left(c_{t}^{-1}\right)^{*} z\right)$, then $\beta_{0} \circ a_{t}=C_{t} \circ b_{0} \circ \beta_{0}$. Since $c_{t}$ is a smooth curve in $\operatorname{Diff}\left(\operatorname{Graph}\left(\beta_{0}\right)\right)$ passing through the identity map, the cotangent lift $C_{t}$ is a smooth curve in $\operatorname{Ham}\left(T^{*} \operatorname{Graph}\left(\beta_{0}\right)\right)$. Thus $j_{t}=\beta_{t} \circ a_{t}=\psi_{t} \circ C_{t} \circ b_{0} \circ \beta_{0}$ lies in the $\operatorname{Ham}\left(T^{*} L_{0}\right)$ orbit through $b_{0} \circ \beta_{0}$, which means the integral manifold $N$ is contained in a $\operatorname{Ham}\left(T^{*} L_{0}\right)$ orbit. For the general case when $M$ is any symplectic manifold, the previous discussion implies that the intersection of any integral manifold with a chart neighbourhood $U_{i}$ on $\operatorname{Lag}\left(L_{0}, M\right)$ lies in a $\operatorname{Ham}(M)$ orbit. Thus any integral manifold containing a point $i \in$ $\operatorname{Lag}\left(L_{0}, M\right)$ is contained in $\operatorname{Ham}(M) \cdot i$, which means that such orbits are maximal integral manifolds.

Finally, we will show that the atlas $\left(U_{i}, \varphi_{i}\right)_{i \in \operatorname{Lag}\left(L_{0}, M\right)}$ consists of distinguished charts. The affine translations of $F$ in $Z^{1}\left(L_{0}\right) \oplus \mathfrak{X}\left(L_{0}\right)$ consist of elements $(\alpha, Y)$ which are pairwise cohomologous in the first factor, i.e $(\alpha, Y) \in\left(\alpha_{0}, Y_{0}\right)+F$ if and only if $\alpha-\alpha_{0} \in B^{1}\left(L_{0}\right)$. Let $\left(U_{i}, \varphi_{i}\right)$ be a chart on $\operatorname{Lag}\left(L_{0}, M\right)$ with corresponding symplectic embedding $\lambda_{i}: T^{*} L_{0} \supset W_{i} \rightarrow M$. The zero section in $T^{*} L_{0}$ can be deformed to the graph of any 1-form $\alpha \in \Omega^{1}\left(L_{0}\right)$ on $L_{0}$ by taking the time 1 flow of the transformation $(x, p) \mapsto\left(x, p+t \alpha_{x}\right)$ of the cotangent bundle. When $\alpha$ is closed this transformation is symplectic; when $\alpha$ is exact it is a Hamiltonian symplectomorphism. So the graph of any exact form can be obtained by deforming the zero section in $T^{*} L_{0}$ along a Hamiltonian vector field. Conversely, suppose that $\phi \in \operatorname{Ham}\left(T^{*} L_{0}\right)$ is a Hamiltonian symplectomorphism and $\left\{\psi_{t}\right\}$ is a collection of symplectomorphisms satisfying $\psi_{0}=\mathrm{Id}, \psi_{1}=\phi$, and $\dot{\psi}_{t}=X_{H_{t}} \circ \psi_{t}$ for some family of Hamiltonian vector fields $X_{H_{t}}$ on $T^{*} L_{0}$. If $\mathbf{O}$ denotes the zero section, then $j_{t}:=\varphi_{i}\left(\lambda_{i} \circ \psi_{t} \circ \mathbf{O}\right)$ is a smooth curve in $Z^{1}\left(L_{0}\right) \oplus \mathfrak{X}\left(L_{0}\right)$ with time derivative equal to $\left(\alpha_{\mathrm{d} \lambda_{i} \circ \dot{\psi}_{t} \circ \mathbf{O}}, \mathrm{d} \pi \circ \dot{\psi}_{t} \circ \mathbf{O}\right)$ for all $t$. Since $\alpha_{\mathrm{d} \lambda_{i} \circ \dot{\psi}_{t} \circ \mathbf{O}}=\left(\psi_{t} \circ \mathbf{O}\right)^{*} d H_{t}$, the curve $j_{t}$ must be contained in $B^{1}\left(L_{0}\right) \oplus \mathfrak{X}\left(L_{0}\right)$, which means $\phi \circ \mathbf{O}=\psi_{1} \circ \mathbf{O}$ is the graph of an exact 1 -form precomposed with a diffeomorphism of $L_{0}$. It follows that two 1-forms are cohomologous if and only if their graphs in $T^{*} L_{0}$ can be joined by flowing along a Hamiltonian vector field. So a curve in $U_{i}$ lies in a $\operatorname{Ham}(M)$ orbit if and only if it is mapped into a translation of $F$ under $\varphi_{i}$. Thus inverse images of translations of $F$ are integral manifolds of $\mathcal{E}$ and intersections of integral manifolds with each chart neighbourhood $U_{i}$ get mapped into translations of $F$ under $\varphi_{i}$.

Definition 1. In the spirit of Weinstein's terminology in [23], we will call the foliation $\mathcal{E}$ the isodrastic foliation of $\operatorname{Lag}\left(L_{0}, M\right)$. An individual leaf of $\mathcal{E}$ will be called an isodrast in $\operatorname{Lag}\left(L_{0}, M\right)$.

The group of orientation preserving diffeomorphisms $\operatorname{Diff}_{+}\left(L_{0}\right)$ acts freely on $\operatorname{Lag}\left(L_{0}, M\right)$ via

$$
a \cdot i:=i \circ a^{-1} \text {. }
$$

The quotient Lag $\left(L_{0}, M\right) / \operatorname{Diff}_{+}\left(L_{0}\right)$ is naturally identified with the set of oriented, compact Lagrangian submanifolds in $M$ diffeomorphic to $L_{0}$.

\section{Notation}

Set

$$
\operatorname{Lag}(M):=\operatorname{Lag}\left(L_{0}, M\right) / \operatorname{Diff}_{+}\left(L_{0}\right) .
$$


Proposition 5. The set $\operatorname{Lag}(M)$ of oriented Lagrangian submanifolds in $M$ diffeomorphic to $L_{0}$ is a smooth manifold modeled on spaces $Z^{1}(L)$ for $L \in \operatorname{Lag}(M)$. The tangent spaces to Lag $(M)$ are given by

$$
T_{L} \operatorname{Lag}(M)=Z^{1}(L)
$$

and for each representative $i \in \operatorname{Lag}\left(L_{0}, M\right)$ in the class $L \in \operatorname{Lag}(M)$,

$$
T_{[i]} \operatorname{Lag}(M)=\Gamma_{\text {closed }}\left(i^{*} T M\right) / \mathfrak{X}\left(L_{0}\right) .
$$

Proof. We will first describe the manifold structure on $\operatorname{Lag}(M)$. For each Lagrangian $L \in$ Lag $(M)$, by Theorem (1) there exists a symplectic embedding $\lambda_{L}: W_{L} \rightarrow T^{*} L$ of an open neighbourhood of $L$ onto an open neighbourhood of the zero section in the cotangent bundle. Define

$$
\begin{aligned}
& U_{L}:=\left\{N \in \operatorname{Lag}(M) \mid N \subset W_{L}, \lambda_{L}(N)=\operatorname{Graph}(\alpha), \alpha \in Z^{1}(L)\right\}, \\
& \varphi_{L}: U_{L} \rightarrow Z^{1}(L), \quad \varphi_{L}(N):=\alpha .
\end{aligned}
$$

The mapping $\varphi_{L}$ maps $U_{L}$ onto the set $\left\{\alpha \in Z^{1}(L) \mid \alpha(L) \subset \lambda_{L}\left(W_{L}\right)\right\}$ which is $c^{\infty}$-open in $Z^{1}(L)$. Thus the collection $\left(U_{L}, \varphi_{L}\right)_{L \in \operatorname{Lag}(M)}$ defines a smooth atlas on $\operatorname{Lag}(M)$ as the transition functions $\varphi_{L N}$ are smooth by smoothness of the symplectic embeddings $\lambda_{L}$.

As for the tangent spaces, suppose that $K_{t}$ is a smooth curve in $\operatorname{Lag}(M)$ such that $K_{0}=L$. If $\left(U_{L^{\prime}}, \varphi_{L^{\prime}}\right)$ is a chart with $L \in U_{L^{\prime}}$, and $\varphi_{L^{\prime}}\left(K_{t}\right)=\operatorname{Graph}\left(\alpha_{t}\right)$ for a smooth curve $\alpha_{t}$ in $Z^{1}(N)$, then the derivative $\left.\frac{d}{d t}\right|_{t=0} \varphi_{N}\left(K_{t}\right) \in Z^{1}\left(L^{\prime}\right)$ canonically defines an element $\beta \in Z^{1}(L)$ via

$$
\alpha_{0}^{*} \beta:=\left.\frac{d}{d t}\right|_{t=0} \varphi_{L^{\prime}}\left(K_{t}\right) .
$$

Thus, $T_{L} \operatorname{Lag}(M) \subset Z^{1}(L)$. Conversely, each $\beta \in Z^{1}(L)$ defines a smooth curve in $\operatorname{Symp}\left(T^{*} L\right)$ via $\psi_{t}(x, p):=(x, p+t \beta(x))$. If $\mathbf{O}$ denotes the zero section of $T^{*} L$, then $\operatorname{Graph}\left(\psi_{t} \circ \mathbf{O}\right)$ is a smooth curve in $\operatorname{Lag}\left(T^{*} L\right)$. If $\lambda_{L}: M \supset W_{L} \rightarrow T^{*} L$ is the symplectic embedding associated to the chart $\left(U_{L}, \varphi_{L}\right)$, then $c(t):=\lambda_{L}^{-1}\left(\operatorname{Graph}\left(\psi_{t} \circ \mathbf{O}\right)\right)$ is a smooth curve in $\operatorname{Lag}(M)$ such that $\left.\frac{d}{d t}\right|_{t=0} \varphi_{L} \circ c(t)=\beta$. So $Z^{1}(L) \subset T_{L} \operatorname{Lag}(M)$.

We will now describe the identification of tangent spaces of $\operatorname{Lag}(M)$ with vector spaces $\Gamma_{\text {closed }}\left(i^{*} T M\right) / \mathfrak{X}\left(L_{0}\right)$. Let $i \in \operatorname{Lag}\left(L_{0}, M\right)$ be a representative in the class $L \in \operatorname{Lag}(M)$. Let $\lambda_{i}: T^{*} L_{0} \supset W_{i} \rightarrow M$ be the symplectic embedding chosen in the definition of the chart $\left(U_{i}, \varphi_{i}\right)$ on $\operatorname{Lag}\left(L_{0}, M\right)$, and $s_{i}: Z^{1}\left(L_{0}\right) \rightarrow \Gamma_{\text {closed }}\left(i^{*} T M\right)$ the corresponding splitting map (see Remark 9). Then the linear map

$$
Z^{1}(L) \rightarrow \Gamma_{\text {closed }}\left(i^{*} T M\right) / \mathfrak{X}\left(L_{0}\right), \quad \alpha \mapsto\left[s_{i}\left(i^{*} \alpha\right)\right]
$$

is a vector space isomorphism.

Proposition 6. The manifold $\operatorname{Lag}\left(L_{0}, M\right)$ is the total space of a principal Diff ${ }_{+}\left(L_{0}\right)$ bundle over Lag $(M)$.

Proof. We begin by describing a fiber bundle atlas. Let $p: \operatorname{Lag}\left(L_{0}, M\right) \rightarrow \operatorname{Lag}(M)$ denote the projection to the quotient. For $i \in \operatorname{Lag}\left(L_{0}, M\right)$ let $\lambda_{i}: T^{*} L_{0} \supset W_{i} \rightarrow M$ be the symplectic embedding chosen in defining the chart $\left(U_{i}, \varphi_{i}\right)$ on $\operatorname{Lag}\left(L_{0}, M\right)$. Define

$$
U_{[i]}:=\left\{N \in \operatorname{Lag}(M) \mid N=\lambda_{i}(\operatorname{Graph}(\alpha)), \alpha \in Z^{1}\left(L_{0}\right), \alpha\left(L_{0}\right) \subset W_{i}\right\} .
$$


Then $p^{-1}\left(U_{[i]}\right)$ consists of all $l \in \operatorname{Lag}\left(L_{0}, M\right)$ such that $l=\lambda_{i} \circ \alpha \circ a$ where $\alpha \in Z^{1}\left(L_{0}\right)$, $\alpha\left(L_{0}\right) \subset W_{i}$, and $a \in \operatorname{Diff}_{+}\left(L_{0}\right)$. Define $\psi_{[i]}: p^{-1}\left(U_{[i]}\right) \rightarrow U_{[i]} \times \operatorname{Diff}_{+}\left(L_{0}\right)$ by

$$
\psi_{[i]}\left(\lambda_{i} \circ \alpha \circ a\right):=\left(\lambda_{i}(\operatorname{Graph}(\alpha)), a\right)
$$

so that the collection $\left(U_{[i]}, \psi_{[i]}\right)_{i \in \operatorname{Lag}\left(L_{0}, M\right)}$ defines a fiber bundle atlas.

$$
\begin{aligned}
& \text { If } N \in U_{[i][j]}:=U_{[i]} \cap U_{[j]} \text {, and } \psi_{[j]}^{-1}(N, a)=\lambda_{j} \circ \alpha \circ a \text { then } \\
& \psi_{[i][j]}(N, a)=\left(N, \pi \circ \lambda_{i}^{-1} \circ \lambda_{j} \circ \alpha \circ a\right) .
\end{aligned}
$$

So if $N \in U_{[i][j]}$ and $N=\lambda_{j}(\operatorname{Graph}(\alpha))$ then define $u_{[i][j]}: U_{[i][j]} \rightarrow \operatorname{Diff}_{+}\left(L_{0}\right)$ by

$$
u_{[i][j]}(N):=\pi \circ \lambda_{i}^{-1} \circ \lambda_{j} \circ \alpha .
$$

It follows that if $N \in U_{[i]} \cap U_{[j]} \cap U_{[k]}$ and $N=\lambda_{j}(\operatorname{Graph}(\alpha))=\lambda_{k}(\operatorname{Graph}(\beta))$ then

$$
\begin{aligned}
& u_{[i][j]}(N) u_{[j][k]}(N)=\pi \circ \lambda_{i}^{-1} \circ \lambda_{j} \circ \alpha \circ \pi \circ \lambda_{j}^{-1} \circ \lambda_{k} \circ \beta=\pi \circ \lambda_{i}^{-1} \circ \lambda_{k} \circ \beta=u_{[i][k]}(N), \\
& u_{[i][i]}(N)=\operatorname{Id}_{L_{0}}, \\
& \psi_{[i][j]}(N, a)=u_{[i][j]} \circ a .
\end{aligned}
$$

So the fiber bundle atlas $\left(U_{[i]}, \psi_{[i]}\right)_{i \in \operatorname{Lag}\left(L_{0}, M\right)}$ and the collection of maps $u_{[i][j]}: U_{[i][j]} \rightarrow$ $\operatorname{Diff}_{+}\left(L_{0}\right)$ define a principal Diff $+\left(L_{0}\right)$ structure.

The group of Hamiltonian symplectomorphisms Ham $(M)$ acts on Lag $(M)$ via

$$
\operatorname{Ham}(M) \circlearrowright \operatorname{Lag}(M): \phi \cdot L:=\phi(L) \text {. }
$$

As in the proof of Proposition 4, the family of subspaces $B^{1}(L) \subset T_{L} \operatorname{Lag}(M)$ and charts $\left(U_{L}, \varphi_{L}\right)$ for $L \in \operatorname{Lag}(M)$ define a foliation $\mathcal{F}$ on $\operatorname{Lag}(M)$. This foliation has finite codimension since the transverse space at each Lagrangian submanifold $L$ is modeled on $H^{1}(L)$.

Definition 2. The foliation $\mathcal{F}$ will be called the isodrastic foliation of $\operatorname{Lag}(M)$, and each leaf of $\mathcal{F}$ will be called an isodrast in $\operatorname{Lag}(M)$.

The foliation $\mathcal{E}$ gives the set $\operatorname{Lag}\left(L_{0}, M\right)$ the structure of a smooth manifold $\operatorname{Lag}\left(L_{0}, M\right)^{\mathcal{E}}$ modeled on spaces $\Gamma_{\text {exact }}\left(i^{*} T M\right)$ for $i \in \operatorname{Lag}\left(L_{0}, M\right)$. Similarly, the foliation $\mathcal{F}$ gives Lag $(M)$ the structure of a smooth manifold $\operatorname{Lag}(M)^{\mathcal{F}}$ modeled on spaces $B^{1}(L)$ for $L \in \operatorname{Lag}(M)$. As in Proposition 6, fiber bundle charts can be chosen to define a principal $\operatorname{Diff}_{+}\left(L_{0}\right)$ bundle $p: \operatorname{Lag}\left(L_{0}, M\right)^{\mathcal{E}} \rightarrow \operatorname{Lag}(M)^{\mathcal{F}}$. This bundle restricts to a principal Diff $+\left(L_{0}\right)$ bundle over each connected component of $\operatorname{Lag}(M)^{\mathcal{F}}$, i.e. over each isodrast in $\operatorname{Lag}(M)$.

\section{Weighted Lagrangian submanifolds}

In this section we introduce the notion of weightings and weighted submanifolds. The set $\operatorname{Lag}_{w}\left(L_{0}, M\right)$ of pairs $(i, \eta)$ consisting of Lagrangian embeddings $i: L_{0} \hookrightarrow M$ and top degree forms $\eta$ that satisfy $\int_{L_{0}} \eta=1$ has the smooth structure of the Cartesian product $\operatorname{Lag}\left(L_{0}, M\right) \times$ $\left\{\eta \in \Omega^{n}\left(L_{0}\right) \mid \int_{L_{0}} \eta=1\right\}$. The foliation $\mathcal{E}$ of $\operatorname{Lag}\left(L_{0}, M\right)$ canonically induces a foliation $\mathcal{E}_{w}$ of $\operatorname{Lag}_{w}\left(L_{0}, M\right)$. The space $\operatorname{Lag}_{w}\left(L_{0}, M\right)$ is the total space of a principal Diff $+\left(L_{0}\right)$ bundle, whose base can be identified with the set $\operatorname{Lag}_{w}(M)$ of Lagrangian submanifolds in $M$ equipped with a top degree form of total measure 1 . The foliation $\mathcal{E}_{w}$ descends to a foliation $\mathcal{F}_{w}$ of the base, so that $\operatorname{Lag}_{w}\left(L_{0}, M\right)^{\mathcal{E}_{w}}$ (cf. Section 2.18) is the total space of a principal Diff $+\left(L_{0}\right)$ bundle over $\operatorname{Lag}_{w}(M)^{\mathcal{F}_{w}}$. On each leaf of $\mathcal{E}_{w}$ we define a 2 -form $\Omega$, basic with respect to this principal group 
action, which descends to a weak symplectic structure on $\operatorname{Lag}_{w}(M)^{\mathcal{F}_{w}}$. Finally, we show that the tangent spaces of $\operatorname{Lag}_{w}(M)$ and of leaves of $\mathcal{F}_{w}$ can be identified with the tangent space descriptions in Weinstein's original construction, and that Weinstein's symplectic structure $\Omega^{W}$ corresponds to our symplectic structure $\Omega$.

Definition 3. A weighting of a compact oriented manifold $L$ is a top degree form $\rho$ on $L$ satisfying $\int_{L} \rho=1$. A pair $(L, \rho)$ will be called a weighted manifold.

\section{Notation}

Let $\Omega_{1}^{n}\left(S_{0}\right)$ denote the set of $n$-forms on a manifold $S_{0}$ that integrate to 1 (where $n=\operatorname{dim} S_{0}$ ), $\Omega_{0}^{n}\left(S_{0}\right)$ the set of $n$-forms on $S_{0}$ that integrate to 0 , and $\operatorname{Lag}_{w}\left(L_{0}, M\right)$ the product $\operatorname{Lag}\left(L_{0}, M\right) \times$ $\Omega_{1}^{n}\left(L_{0}\right)$. That is,

$$
\begin{aligned}
& \Omega_{1}^{n}\left(S_{0}\right):=\left\{\eta \in \Omega^{n}\left(S_{0}\right) \mid \int_{S_{0}} \eta=1\right\}, \\
& \Omega_{0}^{n}\left(S_{0}\right):=\left\{\vartheta \in \Omega^{n}\left(S_{0}\right) \mid \int_{S_{0}} \vartheta=0\right\}, \\
& \operatorname{Lag}_{w}\left(L_{0}, M\right):=\operatorname{Lag}\left(L_{0}, M\right) \times \Omega_{1}^{n}\left(L_{0}\right) .
\end{aligned}
$$

Integration along $L_{0}$ defines a continuous linear functional $\int_{L_{0}}: \Omega^{n}\left(L_{0}\right) \rightarrow \mathbb{R}$ on the convenient vector space $\Omega^{n}\left(L_{0}\right)=\Gamma\left(\bigwedge^{n} T^{*} L_{0}\right)$. So the kernel $\Omega_{0}^{n}\left(L_{0}\right)$ is a $c^{\infty}$-closed (convenient) subspace. The space $\Omega_{1}^{n}\left(L_{0}\right)$ is an affine translation of $\Omega_{0}^{n}\left(L_{0}\right)$, which means it is a smooth manifold modeled on $\Omega_{0}^{n}\left(L_{0}\right)$. So $\operatorname{Lag}_{w}\left(L_{0}, M\right)$ is a smooth manifold modeled on the space $Z^{1}\left(L_{0}\right) \oplus \mathfrak{X}\left(L_{0}\right) \oplus \Omega_{0}^{n}\left(L_{0}\right)$ with the product atlas $\left(U_{(i, \eta)}, \varphi_{(i, \eta)}\right)_{(i, \eta) \in \operatorname{Lag}_{w}\left(L_{0}, M\right)}$. That is, if $\left(U_{i}, \varphi_{i}\right)_{i \in \operatorname{Lag}\left(L_{0}, M\right)}$ is the atlas on $\operatorname{Lag}\left(L_{0}, M\right)$ defined in Proposition 1 then the charts $\left(U_{(i, \eta)}, \varphi_{(i, \eta)}\right)$ are defined by

$$
\begin{aligned}
& U_{(i, \eta)}:=U_{i} \times\left(\eta+\Omega_{0}^{n}\left(L_{0}\right)\right), \\
& \varphi_{(i, \eta)}: U_{(i, \eta)} \rightarrow Z^{1}\left(L_{0}\right) \oplus \mathfrak{X}\left(L_{0}\right) \oplus \Omega_{0}^{n}\left(L_{0}\right), \\
& \varphi_{(i, \eta)}(l, \eta+\vartheta):=\left(\varphi_{i}(l), \vartheta\right)=(\alpha, Y, \vartheta) .
\end{aligned}
$$

This atlas and the subspace $B^{1}\left(L_{0}\right) \oplus \mathfrak{X}\left(L_{0}\right) \oplus \Omega_{0}^{n}\left(L_{0}\right)$ define a foliation $\mathcal{E}_{w}$ on $\operatorname{Lag}_{w}\left(L_{0}, M\right)$.

Definition 4. We will call the foliation $\mathcal{E}_{w}$ the isodrastic foliation of $\operatorname{Lag}_{w}\left(L_{0}, M\right)$ and a leaf of $\mathcal{E}_{w}$ will be called an isodrast in $\operatorname{Lag}_{w}\left(L_{0}, M\right)$.

For each $(i, \eta) \in \operatorname{Lag}_{w}\left(L_{0}, M\right)$, the tangent space $T_{(i, \eta)} \operatorname{Lag}_{w}\left(L_{0}, M\right)$ equals the vector space $\Gamma_{\text {closed }}\left(i^{*} T M\right) \oplus \Omega_{0}^{n}\left(L_{0}\right)$. Meanwhile, the tangent space to an isodrast $\mathbf{H}_{w}$ in $\operatorname{Lag}_{w}\left(L_{0}, M\right)$ at $(i, \eta)$ is given by $T_{(i, \eta)} \mathbf{H}_{w}=\Gamma_{\text {exact }}\left(i^{*} T M\right) \oplus \Omega_{0}^{n}\left(L_{0}\right)$. To each point $(i, \eta) \in \mathbf{H}_{w}$ in an isodrast we assign a skew-symmetric bilinear form on $T_{(i, \eta)} \mathbf{H}_{w}$ via the expression

$$
\Omega_{(i, \eta)}\left(\left(X_{1}, \vartheta_{1}\right),\left(X_{2}, \vartheta_{2}\right)\right):=\int_{L_{0}}\left[\omega\left(X_{1}, X_{2}\right) \eta+h_{1} \vartheta_{2}-h_{2} \vartheta_{1}\right]
$$

where $\alpha_{X_{k}}=d h_{k}$ for some $h_{k} \in C^{\infty}\left(L_{0}, \mathbb{R}\right)$. This assignment does not depend on the choice of primitives $h_{k}$ since the top degree forms $\vartheta_{k}$ integrate to 0 . Equivalently, the pointwise assignment in (3) can be viewed as an assignment on the smooth manifold $\operatorname{Lag}_{w}\left(L_{0}, M\right)^{\mathcal{E}_{w}}$.

Proposition 7. The pointwise assignment in (3) on $\operatorname{Lag}_{w}\left(L_{0}, M\right)^{\mathcal{E}_{w}}$ defines a basic differential 2-form $\Omega$ with respect to the action of $\operatorname{Diff}_{+}\left(L_{0}\right)$ on $\operatorname{Lag}_{w}\left(L_{0}, M\right)^{\mathcal{E}_{w}}$ given by $a \cdot(i, \eta):=$ $\left(i \circ a^{-1},\left(a^{-1}\right)^{*} \eta\right)$. 
Proof. We will first show that the assignment $\Omega$ defines a differential 2 -form on each leaf $\mathbf{H}_{w}$ of $\mathcal{E}_{w}$. The assignment in (3) defines a map $\Omega: \mathbf{H}_{w} \rightarrow L_{\text {alt }}^{2}\left(T \mathbf{H}_{w}, \mathbf{H}_{w} \times \mathbb{R}\right)$. To check that this map is smooth, it is enough to check it in each chart. If $\left(U_{(i, \eta)}, \varphi_{(i, \eta)}\right)$ denotes a chart on $\mathbf{H}_{w}$ then $\Omega$ defines a map from $U_{(i, \eta)}$ to $L_{\text {alt }}^{2}\left(\Gamma_{\text {exact }}\left(i^{*} T M\right) \oplus \Omega_{0}^{n}\left(L_{0}\right), \mathbb{R}\right)\left(\operatorname{after} B^{1}\left(L_{0}\right) \oplus \mathfrak{X}\left(L_{0}\right) \oplus\right.$ $\Omega_{0}^{n}\left(L_{0}\right)$ has been identified with $\Gamma_{\text {exact }}\left(i^{*} T M\right) \times \Omega_{0}^{n}\left(L_{0}\right)$ via the splitting map $s_{i}: Z^{1}\left(L_{0}\right) \rightarrow$ $\Gamma_{\text {closed }}\left(i^{*} T M\right)$ (see Remark 9$)$ ). This map is smooth if it maps smooth curves in $U_{(i, \eta)}$ to smooth curves in $L_{\text {alt }}^{2}\left(\Gamma_{\text {exact }}\left(i^{*} T M\right) \oplus \Omega_{0}^{n}\left(L_{0}\right), \mathbb{R}\right)$. A curve in $L_{\text {alt }}^{2}\left(\Gamma_{\text {exact }}\left(i^{*} T M\right) \oplus \Omega_{0}^{n}\left(L_{0}\right), \mathbb{R}\right)$ is smooth if the induced map $\mathbb{R} \times\left(\Gamma_{\text {exact }}\left(i^{*} T M\right) \oplus \Omega_{0}^{n}\left(L_{0}\right)\right)^{2} \rightarrow \mathbb{R}$ is smooth. Thus to verify that $\Omega$ is smooth, it is enough to check the following statement: If $(M, \omega)=\left(T^{*} L_{0}, \omega_{T^{*} L_{0}}\right), i: L_{0} \hookrightarrow T^{*} L_{0}$ denotes the zero section inclusion, $\left(\alpha_{t} \circ a_{t}, \eta_{t}\right)$ is a smooth curve in $\mathbf{H}_{w},\left(X_{1}(t), \vartheta_{1}(t)\right)$ and $\left(X_{2}(t), \vartheta_{2}(t)\right)$ are smooth curves in $\Gamma_{\text {exact }}\left(i^{*} T\left(T^{*} L_{0}\right)\right) \oplus \Omega_{0}^{n}\left(L_{0}\right)$ satisfying $\alpha_{X_{k}(t)}=d h_{k}(t)$ for smooth curves $h_{k}(t)$ in $C^{\infty}\left(L_{0}, \mathbb{R}\right), Z_{1}(t)$ and $Z_{2}(t)$ are the unique time dependent vector fields on $T^{*} L_{0}$ satisfying $\imath\left(Z_{k}(t)\right) \omega=\pi^{*} \alpha_{X_{k}(t)}$ for all $t, Y_{k}(t):=X_{k}(t)-\left.Z_{k}(t)\right|_{L_{0}} \in \mathfrak{X}\left(L_{0}\right)$, and $s \in C^{\infty}(\mathbb{R}, \mathbb{R})$ is a smooth map, then the map

$$
\begin{aligned}
& \mathbb{R} \rightarrow \mathbb{R}, \\
& \qquad \mapsto \int_{L_{0}}\left[\omega \left(Z_{1}(t) \circ \alpha_{s(t)} \circ a_{s(t)}+\mathrm{d} \alpha_{s(t)} \cdot Y_{1}(t) a_{s(t)},\right.\right. \\
& \left.\left.\quad Z_{2}(t) \circ \alpha_{s(t)} \circ a_{s(t)}+\mathrm{d} \alpha_{s(t)} \cdot Y_{2}(t)_{a_{s(t)}}\right) \eta_{s(t)}+h_{1}(t) \vartheta_{2}(t)-h_{2}(t) \vartheta_{1}(t)\right]
\end{aligned}
$$

is smooth. Since this statement follows from the smoothness of all quantities in the integral, $\Omega$ is indeed a section of $L_{\text {alt }}^{2}\left(T \mathbf{H}_{w}, \mathbf{H}_{w} \times \mathbb{R}\right) \rightarrow \mathbf{H}_{w}$.

We will now show that $\Omega$ is basic with respect to the action of $\operatorname{Diff}_{+}\left(L_{0}\right)$ on $\operatorname{Lag}_{w}\left(L_{0}, M\right)^{\mathcal{E}_{w}}$. If $\left(i_{t}, \eta_{t}\right)$ is a smooth curve in $\operatorname{Lag}_{w}\left(L_{0}, M\right)^{\mathcal{E}_{w}}$ with time derivative $(X, \vartheta) \in \Gamma_{\text {exact }}\left(i^{*} T M\right) \oplus \Omega_{0}^{n}\left(L_{0}\right)$ at $t=0$, then the tangent vector to the curve $l_{a}\left(i_{t}, \eta_{t}\right)=\left(i_{t} \circ a^{-1},\left(a^{-1}\right)^{*} \eta_{t}\right)$ at $t=0$ is given by $\left(X \circ a^{-1},\left(a^{-1}\right)^{*} \vartheta\right)$. Thus,

$$
\begin{aligned}
l_{a}^{*} \Omega_{(i, \eta)}\left(\left(X_{1}, \vartheta_{1}\right),\left(X_{2}, \vartheta_{2}\right)\right)= & {\left[\int_{L_{0}} \omega\left(X_{1} \circ a^{-1}, X_{2} \circ a^{-1}\right)\left(a^{-1}\right)^{*} \eta\right.} \\
& \left.+h_{1} \circ a^{-1}\left(a^{-1}\right) \vartheta_{2}-h_{2} \circ a^{-1}\left(a^{-1}\right)^{*} \vartheta_{1}\right] \\
= & \Omega_{(i, \eta)}\left(\left(X_{1}, \vartheta_{1}\right),\left(X_{2}, \vartheta_{2}\right)\right),
\end{aligned}
$$

which means $\Omega$ is $\operatorname{Diff}_{+}\left(L_{0}\right)$-invariant. To check that $\Omega$ is horizontal, let $Y \in \mathfrak{X}\left(L_{0}\right)$ be in the Lie algebra of Diff ${ }_{+}\left(L_{0}\right)$. If $a_{t}$ is a smooth curve in $\operatorname{Diff}_{+}\left(L_{0}\right)$ through the identity map with time derivative $Y$ at $t=0$, then the generating vector field at a point $(i, \eta) \in \operatorname{Lag}_{w}\left(L_{0}, M\right)$ is given by

$$
Y_{\operatorname{Lag}_{w}\left(L_{0}, M\right)}(i, \eta)=\left.\frac{d}{d t}\right|_{t=0}\left(i \circ a_{t}^{-1},\left(a_{t}^{-1}\right)^{*} \eta\right)=\left(-Y,-\mathcal{L}_{Y} \eta\right) .
$$

Since

$$
\Omega_{(i, \eta)}\left(\left(Y, \mathcal{L}_{Y} \eta\right),\left(X_{2}, \vartheta_{2}\right)\right)=\int_{L_{0}}\left[\omega\left(Y, X_{2}\right) \eta-h_{2} \mathcal{L}_{Y} \eta\right]=0
$$

(after integrating by parts, $\int_{L_{0}} \mathcal{L}_{Y} h_{2} \eta=-\int_{L_{0}} h_{2} \mathcal{L}_{Y} \eta$ ), we conclude that $\Omega$ is also horizontal and thus basic.

The quotient $\operatorname{Lag}_{w}\left(L_{0}, M\right) / \operatorname{Diff}_{+}\left(L_{0}\right)$ is naturally identified with the set of weighted Lagrangian submanifolds. Explicitly, the identification of the quotient $\operatorname{Lag}_{w}\left(L_{0}, M\right) / \operatorname{Diff}_{+}\left(L_{0}\right)$ with the set of pairs $(L, \rho)$ is via the correspondence $[(i, \eta)] \leftrightarrow(L, \rho)$ where $L=i\left(L_{0}\right)$ and $i^{*} \rho=\eta$. 


\section{Notation}

Set

$$
\operatorname{Lag}_{w}(M):=\operatorname{Lag}_{w}\left(L_{0}, M\right) / \operatorname{Diff}_{+}\left(L_{0}\right) .
$$

Proposition 8. $\operatorname{Lag}_{w}(M)$ is a smooth manifold modeled on spaces $Z^{1}(L) \oplus \Omega_{0}^{n}(L)$ for $L \in$ $\operatorname{Lag}(M)$. For each representative $(i, \eta)$ in the class $(L, \rho) \in \operatorname{Lag}_{w}(M)$,

$$
T_{(L, \rho)} \operatorname{Lag}_{w}(M)=\Gamma_{\text {closed }}\left(i^{*} T M\right) \oplus \Omega_{0}^{n}\left(L_{0}\right) /\left\{\left(Y, \mathcal{L}_{Y} \eta\right) \mid Y \in \mathfrak{X}\left(L_{0}\right)\right\} .
$$

Proof. For each $(L, \rho) \in \operatorname{Lag}_{w}(M)$, by Theorem 1 there exists a symplectic embedding $\lambda_{(L, \rho)}$ : $M \supset W_{(L, \rho)} \rightarrow T^{*} L$ of a neighbourhood of $L$ onto a neighbourhood of the zero section in the cotangent bundle. If $\pi_{T^{*} L}: T^{*} L \rightarrow L$ denotes the cotangent bundle projection, then the restriction of $\pi_{T * L}$ to the graph of any 1-form $\alpha \in \Omega^{1}(L)$ in $T^{*} L$ defines a diffeomorphism of that graph onto $L$. Define

$$
\begin{aligned}
& U_{(L, \rho)}:=\left\{(N, \sigma) \in \operatorname{Lag}_{w}(M) \mid N \subset W_{(L, \rho)}, \lambda_{(L, \rho)}(N)=\operatorname{Graph}(\alpha),\right. \\
& \left.\quad \alpha \in Z^{1}(L), \sigma=\lambda_{(L, \rho)}^{*}\left(\left.\pi_{T^{*} L}\right|_{\operatorname{Graph}(\alpha)}\right)^{*}(\rho+\theta), \theta \in \Omega_{0}^{n}(L)\right\}, \\
& \varphi_{(L, \rho)}: U_{(L, \rho)} \rightarrow Z^{1}(L) \oplus \Omega_{0}^{n}(L), \\
& \varphi_{(L, \rho)}(N, \sigma):=(\alpha, \theta) .
\end{aligned}
$$

All chart changings are smooth again by the smoothness of the symplectic embeddings $\lambda_{L}$, so the collection $\left(U_{(L, \rho)}, \varphi_{(L, \rho)}\right)_{(L, \rho) \in \operatorname{Lag}_{w}(M)}$ defines a smooth atlas on $\operatorname{Lag}_{w}(M)$.

We will now describe the identification of tangent spaces of $\operatorname{Lag}_{w}(M)$ with spaces $\Gamma_{\text {closed }}\left(i^{*} T M\right)$ $\oplus \Omega_{0}^{n}\left(L_{0}\right) /\left\{\left(Y, \mathcal{L}_{Y} \eta\right) \mid Y \in \mathfrak{X}\left(L_{0}\right)\right\}$. Let $(i, \eta) \in \operatorname{Lag}_{w}\left(L_{0}, M\right)$ be a representative in the class $(L, \rho) \in \operatorname{Lag}(M)$. Let $\lambda_{i}: T^{*} L_{0} \supset W_{i} \rightarrow M$ denote the symplectic embedding chosen in the definition of the chart $\left(U_{i}, \varphi_{i}\right)$ on $\operatorname{Lag}_{w}\left(L_{0}, M\right)$, and $s_{i}: Z^{1}\left(L_{0}\right) \rightarrow \Gamma_{\text {closed }}\left(i^{*} T M\right)$ the corresponding splitting map. Then the linear map

$$
\begin{aligned}
& T_{[(i, \eta)]} \operatorname{Lag}_{w}(M) \rightarrow \Gamma_{\text {closed }}\left(i^{*} T M\right) \times \Omega_{0}^{n}\left(L_{0}\right) /\left\{\left(Y, \mathcal{L}_{Y} \eta\right) \mid Y \in \mathfrak{X}\left(L_{0}\right)\right\}, \\
& (\alpha, \theta) \mapsto\left[\left(s_{i}\left(i^{*} \alpha\right), \theta\right)\right]
\end{aligned}
$$

is a vector space isomorphism.

The canonical projection $\operatorname{Lag}_{w}(M) \rightarrow \operatorname{Lag}(M)$, which forgets the weightings, pulls back $\mathcal{F}$ to a foliation $\mathcal{F}_{w}$ on $\operatorname{Lag}_{w}(M)$. That is, the collection of subspaces $\left\{B^{1}(L) \oplus \Omega_{0}^{n}(L)\right\}$ and atlas $\left(U_{(L, \rho)}, \varphi_{(L, \rho)}\right)$ indexed by $(L, \rho) \in \operatorname{Lag}_{w}(M)$ define a foliation $\mathcal{F}_{w}$ on $\operatorname{Lag}_{w}(M)$.

Definition 5. The foliation $\mathcal{F}_{w}$ will be called the isodrastic foliation of $\operatorname{Lag}_{w}(M)$ and a leaf $\mathbf{I}_{w}$ of $\mathcal{F}_{w}$ will be called an isodrast in $\operatorname{Lag}_{w}(M)$.

Proposition 9. The smooth manifold $\operatorname{Lag}_{w}\left(L_{0}, M\right)^{\mathcal{E}_{w}}$ is the total space of a principal Diff ${ }_{+}\left(L_{0}\right)$ bundle over $\operatorname{Lag}_{w}(M)^{\mathcal{F}_{w}}$. The basic 2 -form $\Omega$ on $\operatorname{Lag}_{w}\left(L_{0}, M\right)^{\mathcal{E}_{w}}$ descends to a weak symplectic structure on $\operatorname{Lag}_{w}(M)^{\mathcal{F}}$. Thus each isodrast in $\operatorname{Lag}_{w}(M)$ is a weakly symplectic manifold.

Proof. We begin by describing a fiber bundle atlas. For $(i, \eta) \in \operatorname{Lag}_{w}\left(L_{0}, M\right)^{\mathcal{E}_{w}}$, let $\lambda_{(i, \eta)}$ : $T^{*} L_{0} \supset W_{i} \rightarrow M$ denote the symplectic embedding chosen in defining the chart $\left(U_{(i, \eta)}, \varphi_{(i, \eta)}\right)$. Define

$$
\begin{aligned}
U_{[(i, \eta)]}:=\{ & (N, \sigma) \in \operatorname{Lag}_{w}(M) \mid N=\lambda_{(i, \eta)}(\operatorname{Graph}(\alpha)), \\
& \left.\alpha \in B^{1}\left(L_{0}\right), \sigma=\left.\lambda_{(i, \eta)}^{-1}\right|_{N} ^{*} \pi^{*}\left(i^{*} \rho+\vartheta\right), \vartheta \in \Omega_{0}^{n}\left(L_{0}\right)\right\} .
\end{aligned}
$$


If $p: \operatorname{Lag}_{w}\left(L_{0}, M\right)^{\mathcal{E}_{w}} \rightarrow \operatorname{Lag}_{w}(M)^{\mathcal{F}_{w}}$ denotes the projection to the quotient, then

$$
\begin{aligned}
p^{-1}\left(U_{[(i, \eta)]}\right)=\{ & (l, \kappa) \in \operatorname{Lag}_{w}\left(L_{0}, M\right)^{\mathcal{E}_{w}} \mid l=\lambda_{(i, \eta)} \circ \alpha \circ a, \alpha \in B^{1}\left(L_{0}\right), \\
& \left.\alpha\left(L_{0}\right) \subset W_{i}, a \in \operatorname{Diff}_{+}\left(L_{0}\right)\right\} .
\end{aligned}
$$

Define $\psi_{[(i, \eta)]}: p^{-1}\left(U_{[(i, \eta)]}\right) \rightarrow U_{[(i, \eta)]} \times \operatorname{Diff}_{+}\left(L_{0}\right)$ by

$$
\psi_{[(i, \eta)]}\left(\left(\lambda_{(i, \eta)} \circ \alpha \circ a, \kappa\right)\right):=\left(\left(\lambda_{(i, \eta)}(\operatorname{Graph}(\alpha)),\left.\lambda_{(i, \eta)}^{-1}\right|_{N} ^{*} \pi^{*}\left(a^{-1}\right)^{*} \kappa\right), a\right)
$$

so that the collection $\left(U_{[(i, \eta)]}, \psi_{[(i, \eta)]}\right)_{(i, \eta) \in \operatorname{Lag}_{w}\left(L_{0}, M\right)}$ defines a fiber bundle atlas.

$$
\text { If }(N, \sigma) \in U_{[(i, \eta)][(j, \nu)]} \text {, and } \psi_{[(j, \nu)]}^{-1}((N, \sigma), a)=\left(\lambda_{j} \circ \alpha \circ a, \kappa\right) \text { then }
$$

$$
\psi_{[(i, \eta)][(j, \nu)]}((N, \sigma), a)=\left((N, \sigma), \pi \circ \lambda_{(i, \eta)}^{-1} \circ \lambda_{(j, \nu)} \circ \alpha \circ a\right) .
$$

So if $(N, \sigma) \in U_{[(i, \eta)][(j, \nu)]}$ and $N=\lambda_{j}(\operatorname{Graph}(\alpha))$ then define $u_{[i][j]}: U_{[(i, \eta)][(j, \nu)]} \rightarrow \operatorname{Diff}_{+}\left(L_{0}\right)$ by

$$
u_{[i][j]}((N, \sigma)):=\pi \circ \lambda_{(i, \eta)}^{-1} \circ \lambda_{(j, \nu)} \circ \alpha .
$$

It follows that if $(N, \sigma) \in U_{[(i, \eta)]} \cap U_{[(j, \nu)]} \cap U_{[(k, \mu)]}, N=\lambda_{(j, \nu)}(\operatorname{Graph}(\alpha))$, and $N=\lambda_{(k, \mu)}(\operatorname{Graph}(\beta))$ then

$$
\begin{aligned}
& u_{[i][j]}((N, \sigma)) u_{[j][k]}((N, \sigma))=\pi \circ \lambda_{i}^{-1} \circ \lambda_{j} \circ \alpha \circ \pi \circ \lambda_{j}^{-1} \circ \lambda_{k} \circ \beta \\
& =\pi \circ \lambda_{i}^{-1} \circ \lambda_{k} \circ \beta=u_{[i][k]}((N, \sigma)), \\
& u_{[i][i]}((N, \sigma))=\operatorname{Id}_{L_{0}}, \\
& \psi_{[(i, \eta)][(j, \nu)]}((N, \sigma), a)=u_{[(i, \eta)][(j, \nu)]} \circ a \text {. }
\end{aligned}
$$

Hence, the fiber bundle atlas $\left(U_{[(i, \eta)]}, \psi_{[(i, \eta)]}\right)_{(i, \eta) \in \operatorname{Lag}_{w}\left(L_{0}, M\right)}$ and the collection of maps $u_{[(i, \eta)][(j, \nu)]}$ : $U_{[(i, \eta)][(j, \nu)]} \rightarrow \operatorname{Diff}_{+}\left(L_{0}\right)$ define a principal Diff ${ }_{+}\left(L_{0}\right)$ structure.

Since $\Omega$ defines a basic 2 -form on the total $\operatorname{space}_{\operatorname{Lag}_{w}}\left(L_{0}, M\right)^{\mathcal{E}_{w}}$, it descends to a differential 2 -form (also denoted $\Omega$ ) on $\operatorname{Lag}_{w}(M)^{\mathcal{F}_{w}}$ (see Section 2.34).

We will now check closedness of $\Omega$ locally in a chart $\left(U_{(L, \rho)}, \varphi_{(L, \rho)}\right)$. On $U_{(L, \rho)}$ tangent vectors can be identified with pairs $(Z, \vartheta)$ where $Z \in \mathfrak{X}\left(T^{*} L_{0}\right)$ is a vector field on the cotangent bundle satisfying $\imath(Z) \omega=\pi^{*} d h$ for $h \in C^{\infty}\left(L_{0}, \mathbb{R}\right)$, and $\vartheta \in \Omega_{0}^{n}\left(L_{0}\right)$. Under such an identification, if $(i, \eta)$ is a representative in the class $(L, \rho) \in \operatorname{Lag}_{w}(M)$, and if we identify $M$ with $T^{*} L_{0}$ using the symplectic embedding $\lambda_{i}: T^{*} L_{0} \supset W_{i} \rightarrow M$, then terms like $\omega\left(X_{1}, X_{2}\right) \eta=i^{*}\left[\omega\left(Z_{1}, Z_{2}\right)\right] \eta$ in the expression for $\Omega$ vanish since the $Z_{k}$ 's are tangent to the cotangent fibers. So on $U_{(L, \rho)}$,

$$
\Omega_{(N, \sigma)}\left(\left(X_{1}, \vartheta_{1}\right),\left(X_{2}, \vartheta_{2}\right)\right)=\int_{L_{0}}\left(h_{1} \vartheta_{2}-h_{2} \vartheta_{1}\right)
$$

It follows that $d \Omega=0$ since locally $\Omega$ does not depend on $(N, \sigma)$.

Finally, weak nondegeneracy follows from the local expression for $\Omega$ in (4) and the fact that the $h_{k}$ 's and $\vartheta_{k}$ 's can be chosen independently. Indeed, if $h_{1}$ is nonzero on some open subset $V \subset L_{0}$, then we can take $h_{2}$ to be zero and choose $\vartheta_{2}$ such that it is supported on $V$ and $\int_{L_{0}} h_{1} \vartheta_{2}$ is nonzero. If $\vartheta_{1}$ is nonzero on an open subset $V \subset L_{0}$, then we can choose $\vartheta_{2}$ to be zero and choose $h_{2}$ to be supported on $V$ so that $\int_{L_{0}} h_{2} \vartheta_{1}$ is nonzero. 
$\operatorname{Lag}_{w}(M)$ can also be described as the set of equivalence classes $(L,[\widetilde{\rho}])$ where $\widetilde{\rho}$ is an $n$-form on a neighbourhood of $L$ satisfying $\int_{L} \widetilde{\rho}=1$, and $\widetilde{\rho}_{1} \sim \widetilde{\rho}_{2}$ if and only if $\widetilde{\rho}_{1}$ and $\widetilde{\rho}_{2}$ have the same pullback to $L$. In [23] Weinstein used this approach and heuristically viewed $\operatorname{Lag}_{w}(M)$ and each leaf $\mathbf{I}_{w}$ of $\mathcal{F}_{w}$ as infinite dimensional manifolds. He viewed tangent vectors as equivalence classes of paths in $\operatorname{Lag}_{w}(M)$ and $\mathbf{I}_{w}$ to give the following description of their tangent spaces and wrote down a closed, weakly nondegenerate, skew-symmetric bilinear form $\Omega^{W}$ on each isodrast $\mathbf{I}_{w}$ :

Theorem 2 (see Theorem $3.2 \&$ Lemma 3.3 in [23]). The tangent space to $\operatorname{Lag}_{w}(M)$ at $(L, \rho)$ can be identified with the set of quadruples $(L, \widetilde{\rho}, X, \widetilde{\theta})$, where $\widetilde{\rho}$ is an $n$-form on a neighbourhood of $L$ satisfying $\int_{L} \widetilde{\rho}=1, X$ is a symplectic vector field on a neighbourhood of $L$, and $\widetilde{\theta}$ is an $n$-form on a neighbourhood of L satisfying $\int_{L} L_{X} \widetilde{\rho}+\widetilde{\theta}=0$, subject to the following equivalence relation. $\left(L, \widetilde{\rho}_{1}, X_{1}, \widetilde{\theta}_{1}\right) \sim\left(L, \widetilde{\rho}_{2}, X_{2}, \widetilde{\theta}_{2}\right)$ if and only if the following conditions hold: (1) $\widetilde{\rho}_{1}$ and $\widetilde{\rho}_{2}$ have the same pullback to $L ;(2) X_{1}-X_{2}$ is tangent to $L$; (3) the pullbacks to $L$ of $L_{X_{1}} \widetilde{\rho}_{1}+\widetilde{\theta}_{1}$ and $L_{X_{1}} \widetilde{\rho}_{2}+\widetilde{\theta}_{2}$ are equal.

The tangent vectors to an isodrast $\mathbf{I}_{w}$ are represented by equivalence classes $\left[\left(L, \widetilde{\rho}, X_{f}, \widetilde{\theta}\right)\right]$ where $X_{f}$ is a Hamiltonian vector field on a neighbourhood of $L . \mathbf{I}_{w}$ admits a closed, weakly nondegenerate, skew-symmetric bilinear form $\Omega^{W}$ defined by

$$
\Omega_{(L, \rho)}^{W}\left(\xi_{1}, \xi_{2}\right):=\int_{L}\left[\left\{f_{1}, f_{2}\right\} \rho+f_{1}\left(L_{X_{f_{2}}} \widetilde{\rho}_{2}+\widetilde{\theta}_{2}\right)-f_{2}\left(L_{X_{f_{1}}} \widetilde{\rho}_{1}+\widetilde{\theta}_{1}\right)\right] .
$$

We will show that this heuristic description of the tangent spaces and bilinear structure $\Omega^{W}$ due to Weinstein can be derived from the smooth structures on $\operatorname{Lag}_{w}(M)$ and $\mathbf{I}_{w}$ defined in Proposition 8 and from the weak symplectic structure $\Omega$ on $\mathbf{I}_{w}$ (see (3)).

\section{Notation}

For $(L, \rho) \in \operatorname{Lag}_{w}(M)$ let $Q_{(L, \rho)}^{\text {symp }}$ denote the space of equivalence classes $[(L, \widetilde{\rho}, X, \widetilde{\theta})]$ where $X$ is a symplectic vector field defined on a neighbourhood of $L$. Let $Q_{(L, \rho)}^{\text {ham }}$ denote the space of equivalence classes $\left[\left(L, \widetilde{\rho}, X_{f}, \widetilde{\theta}\right)\right]$ where $X_{f}$ is a Hamiltonian vector field defined on a neighbourhood of $L$.

Proposition 10. For every representative $(i, \eta) \in \operatorname{Lag}_{w}\left(L_{0}, M\right)$ in the class $(L, \rho) \in \operatorname{Lag}_{w}(M)$ there exists a vector space isomorphism

$$
\tau_{(i, \eta)}^{\mathrm{symp}}: T_{(L, \rho)} \operatorname{Lag}_{w}(M)=\Gamma_{\text {closed }}\left(i^{*} T M\right) \times \Omega_{0}^{n}\left(L_{0}\right) /\left\{\left(Y, \mathcal{L}_{Y} \eta\right) \mid Y \in \mathfrak{X}\left(L_{0}\right)\right\} \rightarrow Q_{(L, \rho)}^{\mathrm{symp}} .
$$

For every representative $(i, \eta) \in \operatorname{Lag}_{w}\left(L_{0}, M\right)$ in the class $(L, \rho)$ in an isodrast $\mathbf{I}_{w}$, this restricts to a vector space isomorphism

$$
\tau_{(i, \eta)}^{\text {ham }}: T_{(L, \rho)} \mathbf{I}_{w}=\Gamma_{\text {exact }}\left(i^{*} T M\right) \times \Omega_{0}^{n}\left(L_{0}\right) /\left\{\left(Y, \mathcal{L}_{Y} \eta\right) \mid Y \in \mathfrak{X}\left(L_{0}\right)\right\} \rightarrow Q_{(L, \rho)}^{\text {ham }} .
$$

Under the induced vector space identification $T_{(L, \rho)} \mathbf{I}_{w} \simeq Q_{(L, \rho)}^{\text {ham }}$, if $\zeta_{1}, \zeta_{2} \in T_{(L, \rho)} \mathbf{I}_{w}$ correspond to $\xi_{1}, \xi_{2} \in Q_{(L, \rho)}^{\text {ham }}$ then $\Omega_{(L, \rho)}\left(\zeta_{1}, \zeta_{2}\right)=\Omega_{(L, \rho)}^{W}\left(\xi_{1}, \xi_{2}\right)$.

Proof. For $(L, \rho)=[(i, \eta)] \in \operatorname{Lag}_{w}(M)$ let $\lambda_{(i, \eta)}: T^{*} L_{0} \supset W_{(i, \eta)} \rightarrow M$ denote the symplectic embedding chosen for the chart $\left(U_{(i, \eta)}, \varphi_{(i, \eta)}\right)$ on $\operatorname{Lag}_{w}\left(L_{0}, M\right)$. Let $[(X, \vartheta)] \in T_{(L, \rho)} \operatorname{Lag}_{w}(M)$ be a tangent vector, with representative $(X, \vartheta)$ such that $\mathrm{d} \lambda_{(i, \eta)}^{-1} \circ X$ is tangent to the cotangent fibers in $T^{*} L_{0}$. Let $Z_{\alpha_{X}}$ be the unique vector field on $T^{*} L_{0}$ satisfying $\imath\left(Z_{\alpha_{X}}\right) \omega_{T^{*} L_{0}}=\pi^{*} \alpha_{X}$. Set $\widetilde{\rho}:=\left(\lambda_{(i, \eta)}^{-1}\right)^{*} \pi^{*} i^{*} \rho, \widetilde{Z}_{\alpha_{X}}:=\mathrm{d} \lambda_{(i, \eta)}\left(Z_{\alpha_{X}}\right)$ and $\widetilde{\vartheta}:=\left(\lambda_{(i, \eta)}^{-1}\right)^{*} \pi^{*} \vartheta$, and define

$$
\tau_{(L, \rho)}^{\mathrm{symp}}: T_{(L, \rho)} \operatorname{Lag}_{w}(M) \rightarrow Q_{(L, \rho)}^{\mathrm{symp}}, \quad \tau_{(L, \rho)}^{\mathrm{symp}}([(X, \vartheta)]):=\left[\left(L, \widetilde{\rho}, \widetilde{Z}_{\alpha_{X}}, \widetilde{\vartheta}\right)\right] .
$$




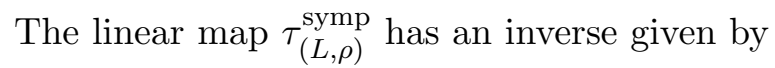

$$
Q_{(L, \rho)}^{\mathrm{symp}} \rightarrow T_{(L, \rho)} \operatorname{Lag}_{w}(M), \quad[(L, \widetilde{\rho}, \tilde{X}, \tilde{\vartheta})] \mapsto\left[\left(\tilde{X} \circ i, i^{*}\left(L_{\tilde{X}} \widetilde{\rho}+\widetilde{\vartheta}\right)\right)\right] .
$$

The isomorphism $\tau_{(L, \rho)}^{\text {ham }}: T_{(L, \rho)} \mathbf{I}_{w} \rightarrow Q_{(L, \rho)}^{\text {ham }}$ is described similarly.

Finally if $\zeta_{1}, \zeta_{2} \in T_{(L, \rho)} \mathbf{I}_{w}$ with $\zeta_{k}=\left[\left(X_{k}, \vartheta_{k}\right)\right]$, with representatives $\left(X_{k}, \vartheta_{k}\right)$ such that $\mathrm{d} \lambda_{(i, \eta)}^{-1} \circ X_{k}$ is tangent to the cotangent fibers in $T^{*} L_{0}$, then $\omega\left(X_{1}, X_{2}\right)=0$. So if $\alpha_{X_{k}}=d h_{k}$, $\widetilde{h}_{k}:=\left(\lambda_{(i, \eta)}^{-1}\right)^{*} \pi^{*} h_{k}$, and $\widetilde{\vartheta}_{k}:=\left(\lambda_{(i, \eta)}^{-1}\right)^{*} \pi^{*} \vartheta_{k}$ then

$$
\Omega_{(L, \rho)}^{W}\left(\tau_{(L, \rho)}^{\mathrm{ham}}\left(\zeta_{1}\right), \tau_{(L, \rho)}^{\mathrm{ham}}\left(\zeta_{2}\right)\right)=\int_{L}\left(\widetilde{h}_{1} \widetilde{\vartheta}_{2}-\widetilde{h}_{2} \widetilde{\vartheta}_{1}\right)=\int_{L_{0}}\left(h_{1} \vartheta_{2}-h_{2} \vartheta_{1}\right)=\Omega_{(L, \rho)}\left(\zeta_{1}, \zeta_{2}\right) .
$$

Example 1. Let $M=S^{2}$ and $L_{0}=S^{1}$. Since $S^{1}$ is one dimensional, all embeddings are Lagrangian and all 1-forms on $S^{1}$ are closed. So $\operatorname{Lag}\left(S^{1}, S^{2}\right)=\operatorname{Emb}\left(S^{1}, S^{2}\right)$ and for every embedding $i$ we have that $T_{i} \operatorname{Lag}\left(S^{1}, S^{2}\right)=\Gamma\left(i^{*} T S^{2}\right)$.

For any point $[(i, \eta)]=(L, \rho)$ in a leaf $\mathbf{I}_{w}$, if $j$ is a compatible almost complex structure on $S^{2}$, i.e. $g(\cdot, \cdot):=\omega_{S^{2}}(\cdot, j \cdot)$ defines a Riemannian metric on $S^{2}$, then for every tangent vector $\xi \in T_{[(i, \eta)]} \mathbf{I}_{w}$ there exists a unique representative $(X, \vartheta) \in \xi$ with $X(x) \in j T_{i(x)} L$ for every $x \in L_{0}$. For such choices of representatives the expression for $\Omega$ becomes

$$
\Omega\left(\left[\left(X_{1}, \vartheta_{1}\right)\right],\left[\left(X_{2}, \vartheta_{2}\right)\right]\right)=\int_{S^{1}}\left(h_{1} \vartheta_{2}-h_{2} \vartheta_{1}\right)
$$

where $\alpha_{X_{k}}=d h_{k}$ for $h_{k} \in C^{\infty}\left(S^{1}, \mathbb{R}\right)$ (because the subspaces $j T_{i(x)} L \subset T_{i(x)} S^{2}$ are Lagrangian).

Each $\vartheta_{k}$ can be written as $r_{k}(x) d x$ for some function $r_{k}$ on $S^{1}$. Meanwhile, any function $f$ on $S^{1}$ has a Fourier series expansion

$$
f(x)=\sum_{n=-\infty}^{\infty} \widehat{f}(n) e^{i n \pi x}
$$

which reduces the expression for $\Omega$ to

$$
\Omega\left(\xi_{1}, \xi_{2}\right)=\sum_{n=-\infty}^{\infty}\left(\widehat{h}_{1}(n) \widehat{\vartheta}_{2}(n)-\widehat{h}_{2}(n) \widehat{\vartheta}_{1}(n)\right) .
$$

This expression is a countably infinite version of the standard symplectic vector space structure.

Remark 10. Weinstein's original construction was more general than we have described so far. It included the case of Lagrangian submanifolds which are neither compact nor oriented. In this case Weinstein used compactly supported densities instead of volume forms. All of our constructions also carry through for non-oriented Lagrangian submanifolds equipped with compactly supported densities.

Example 2. Let $(M, \omega)=\left(\mathbb{R}^{2}, d q \wedge d p\right)$ and $L_{0}=\mathbb{R}$. As in Ex. $1, \mathbb{R}$ is one dimensional which means $\operatorname{Lag}\left(\mathbb{R}, \mathbb{R}^{2}\right)=\operatorname{Emb}\left(\mathbb{R}, \mathbb{R}^{2}\right)$ and for every embedding $i$ we have that $T_{i} \operatorname{Lag}\left(L_{0}, M\right)=$ $\Gamma\left(i^{*} T M\right)$. Moreover, since $H^{1}\left(L_{0}\right)=0$ the leaves of $\mathcal{E}$ consist of path connected components in $\operatorname{Emb}\left(\mathbb{R}, \mathbb{R}^{2}\right)$. Thus the leaves of $\mathcal{F}$ consist of oriented one dimensional submanifolds in $\mathbb{R}^{2}$ diffeomorphic to $\mathbb{R}$.

Though $L_{0}$ is not compact, we can still use compactly supported 1-forms as weightings. A leaf $\mathbf{I}_{w}$ then of $\mathcal{F}_{w}$ consists of isotopic one dimensional submanifolds in $\mathbb{R}^{2}$ diffeomorphic to $\mathbb{R}$, equipped with compactly supported 1-forms. Any tangent vector $X \in T_{i} \operatorname{Lag}\left(\mathbb{R}, \mathbb{R}^{2}\right)$ can be written in components as $X=\widetilde{q} \frac{\partial}{\partial q}+\widetilde{p} \frac{\partial}{\partial p}$. Since any 1 -form $\eta$ on $\mathbb{R}$ can be written as $\widetilde{\eta}(x) d x$ for some function $\tilde{\eta}$, the expression for $\Omega$ on such a leaf $\mathbf{I}_{w}$ becomes

$$
\Omega\left(\xi_{1}, \xi_{2}\right)=\int_{-\infty}^{\infty}\left(\widetilde{q}_{1}(x) \widetilde{p}_{2}(x) \widetilde{\eta}-\widetilde{q}_{2}(x) \widetilde{p}_{1}(x) \widetilde{\eta}+\widetilde{h}_{1} \widetilde{\vartheta}_{2}-\widetilde{h}_{2} \widetilde{\vartheta}_{1}\right) d x
$$




\section{Positive weighted Lagrangian submanifolds}

In this section we will consider an open subset $\operatorname{Lag}_{p w}(M)$ of $\operatorname{Lag}_{w}(M)$ consisting of Lagrangian submanifolds weighted with volume forms. All constructions involving not necessarily positive weightings from before carry over to this case. In particular there is a foliation $\mathcal{F}_{p w}$ of $\operatorname{Lag}_{p w}(M)$ whose leaves have a weak symplectic structure. The $\operatorname{space}^{\operatorname{Lag}_{p w}}(M)$ also has a different description. By fixing a positive weighting $\eta_{0}$, the space of positive weighted Lagrangian submanifolds can be identified with the quotient of $\operatorname{Lag}\left(L_{0}, M\right)$ by the group of diffeomorphisms that preserve $\eta_{0}$. This identification is $\operatorname{Ham}(M)$ equivariant and makes use of Moser's Lemma [10].

Fix $L_{0}$ to be a compact oriented manifold and $(M, \omega)$ a symplectic manifold with $\operatorname{dim} L_{0}=$ $\frac{1}{2} \operatorname{dim} M$ as before.

\section{Notation}

Let $\operatorname{Vol}_{1}\left(S_{0}\right)$ denote the set of volume forms on a compact oriented manifold $S_{0}$ that integrate to 1 and $\operatorname{Lag}_{p w}\left(L_{0}, M\right)$ the product $\operatorname{Lag}\left(L_{0}, M\right) \times \operatorname{Vol}_{1}\left(L_{0}\right)$. That is,

$$
\begin{aligned}
& \operatorname{Vol}_{1}\left(S_{0}\right):=\left\{\eta \in \Omega^{n}\left(S_{0}\right) \mid \eta \text { nowhere vanishing, } \int_{S_{0}} \eta=1\right\}, \\
& \operatorname{Lag}_{p w}\left(L_{0}, M\right):=\operatorname{Lag}\left(L_{0}, M\right) \times \operatorname{Vol}_{1}\left(L_{0}\right) .
\end{aligned}
$$

For every $\eta \in \operatorname{Vol}_{1}\left(L_{0}\right)$, there exists a $c^{\infty}$-open neighbourhood $U_{\eta}$ of $0 \in \Omega_{0}^{n}\left(L_{0}\right)$ such that $\eta+U_{\eta} \subset \operatorname{Vol}_{1}\left(L_{0}\right)$. Thus $\operatorname{Vol}_{1}\left(L_{0}\right)$ is a $c^{\infty}$-open subset of the affine space $\Omega_{1}^{n}\left(L_{0}\right)$. It follows that $\operatorname{Lag}\left(L_{0}, M\right) \times \operatorname{Vol}_{1}\left(L_{0}\right)$ is a smooth manifold with an atlas $\left(U_{(i, \eta)}, \varphi_{(i, \eta)}\right){ }_{(i, \eta) \in \operatorname{Lag}_{p w}\left(L_{0}, M\right)}$ given by (2) except that now

$$
U_{(i, \eta)}=U_{i} \times\left(\eta+U_{\eta}\right)
$$

The atlas $\left(U_{(i, \eta)}, \varphi_{(i, \eta)}\right)_{(i, \eta) \in \operatorname{Lag}_{p w}\left(L_{0}, M\right)}$ and the subspace $B^{1}\left(L_{0}\right) \oplus \mathfrak{X}\left(L_{0}\right) \oplus \Omega_{0}^{n}\left(L_{0}\right)$ define a foliation $\mathcal{E}_{p w}$ on $\operatorname{Lag}_{p w}\left(L_{0}, M\right)$.

Definition 6. We will call $\mathcal{E}_{p w}$ the isodrastic foliation of $\operatorname{Lag}_{p w}\left(L_{0}, M\right)$ and a leaf of $\mathcal{E}_{p w}$ will be called an isodrast in $\operatorname{Lag}_{p w}\left(L_{0}, M\right)$.

The quotient of $\operatorname{Lag}_{p w}\left(L_{0}, M\right)$ by the $\operatorname{Diff}_{+}\left(L_{0}\right)$ action

$$
a \cdot(i, \eta):=\left(i \circ a^{-1},\left(a^{-1}\right)^{*} \eta\right)
$$

can be identified with the set of oriented Lagrangian submanifolds in $M$ equipped with positive weightings.

\section{Notation}

Set

$$
\operatorname{Lag}_{p w}(M):=\operatorname{Lag}_{p w}\left(L_{0}, M\right) / \operatorname{Diff}_{+}\left(L_{0}\right) .
$$

As in Proposition 8, the set $\operatorname{Lag}_{p w}(M)$ admits an atlas of charts $\left(U_{(L, \rho)}, \varphi_{(L, \rho)}\right)$ indexed by $(L, \rho) \in \operatorname{Lag}_{p w}(M)$ modeled on spaces $Z^{1}(L) \oplus \Omega_{0}^{n}(L)$ for $L \in \operatorname{Lag}(M)$. For each representative $(i, \eta)$ in the class $(L, \rho) \in \operatorname{Lag}_{p w}(M)$ the tangent space to $\operatorname{Lag}_{p w}(M)$ at $(L, \rho)$ is equal to $\Gamma_{\text {closed }}\left(i^{*} T M\right) \oplus \Omega_{0}^{n}\left(L_{0}\right) /\left\{\left(Y, \mathcal{L}_{Y} \eta\right) \mid Y \in \mathfrak{X}\left(L_{0}\right)\right\}$. The canonical projection $\operatorname{Lag}_{p w}(M) \rightarrow$ $\operatorname{Lag}(M):(L, \rho) \mapsto L$ pulls back the foliation $\mathcal{F}$ on $\operatorname{Lag}(M)$ to a foliation $\mathcal{F}_{p w}$ on $\operatorname{Lag}_{p w}(M)$. 
Definition 7. We will call $\mathcal{F}_{p w}$ the isodrastic foliation of $\operatorname{Lag}_{p w}(M)$ and a leaf of $\mathcal{F}_{p w}$ will be called an isodrast in $\operatorname{Lag}_{p w}(M)$.

Using a result of Moser [10], we can describe isodrasts in $\operatorname{Lag}_{p w}(M)$ more explicitly. Moser's Lemma states that if $\Lambda_{0}$ and $\Lambda_{1}$ are two volume forms on a compact manifold $N$, such that $\int_{N} \Lambda_{0}=\int_{N} \Lambda_{1}$, then there exists an isotopy $\psi_{t} \in \operatorname{Diff}_{+}(N)$ satisfying $\psi_{1}^{*} \Lambda_{0}=\Lambda_{1}$. Thus a positive weighting on a Lagrangian submanifold $L$ can be moved to any other positive weighting via an isotopy of $L$. Any such isotopy $\psi_{t}$ can be lifted to a Hamiltonian isotopy of $M$ in the following way. Choose a symplectic embedding $\lambda: M \supset U \rightarrow T^{*} L$ of a neighbourhood $U$ of $L$ onto a neighbourhood of the zero section in $T^{*} L$. If $\psi_{t}^{\sharp}$ denotes the cotangent lift of $\psi_{t}$, i.e. $\psi_{t}^{\sharp}(x, p):=\left(\psi_{t}(x),\left(\psi_{t}^{-1}\right)^{*} p\right)$, then $\tau_{t}:=\lambda^{-1} \circ \psi_{t}^{\sharp} \circ \lambda$ extends $\psi_{t}$ to a Hamiltonian isotopy of a neighbourhood of $L$. Let $H_{t}$ be a time dependent family of Hamiltonians corresponding to $\tau_{t}$. If $\chi$ is a cutoff function supported near $L$, and equal to 1 near $L$, then the family $\chi H_{t}$ generates a Hamiltonian isotopy of $M$ which restricts to $\psi_{t}$ on $L$. It follows that the isodrasts in $\operatorname{Lag}_{p w}(M)$ are equal to the $\operatorname{Ham}(M)$ orbits in $\operatorname{Lag}_{p w}(M)$ under the action

$$
\phi \cdot[(i, \eta)]:=[(\phi \circ i, \eta)] \leftrightarrow \phi \cdot(L, \rho):=\left(\phi(L),\left(\phi^{-1}\right)^{*} \rho\right) .
$$

We can also describe $\operatorname{Lag}_{p w}(M)$ and each leaf $\mathbf{I}_{w} \subset \operatorname{Lag}_{p w}(M)$ in a different way. Suppose that $L_{0}$ is equipped with a fixed volume form $\eta_{0}$ that integrates to 1 .

\section{Notation}

Let $\operatorname{Diff}\left(S_{0}, \eta_{0}\right)$ denote the group of diffeomorphisms of a manifold $S_{0}$ that preserve a fixed volume form $\eta_{0}$, and $\mathfrak{X}\left(S_{0}, \eta_{0}\right)$ the set of divergence free vector fields on $S_{0}$. That is,

$$
\begin{aligned}
& \operatorname{Diff}\left(S_{0}, \eta_{0}\right):=\left\{a \in \operatorname{Diff}\left(S_{0}\right) \mid a^{*} \eta_{0}=\eta_{0}\right\}, \\
& \mathfrak{X}\left(S_{0}, \eta_{0}\right):=\left\{\xi \in \mathfrak{X}\left(S_{0}\right) \mid \mathcal{L}_{\xi} \eta_{0}=0\right\} .
\end{aligned}
$$

The space $\mathfrak{X}\left(S_{0}, \eta_{0}\right)$ is the Lie algebra of $\operatorname{Diff}\left(S_{0}, \eta_{0}\right)$ (see Theorem 43.7 in [9]). Meanwhile, $\operatorname{Diff}\left(L_{0}, \eta_{0}\right)$ acts freely on $\operatorname{Lag}\left(L_{0}, M\right)$ via

$$
a \cdot i:=i \circ a^{-1} \text {. }
$$

Proposition 11. The quotient space $\operatorname{Lag}\left(L_{0}, M\right) / \operatorname{Diff}\left(L_{0}, \eta_{0}\right)$ is a smooth manifold modeled on spaces $\Gamma_{\text {closed }}\left(i^{*} T M\right) / \mathfrak{X}\left(L_{0}, \eta_{0}\right)$ for $i \in \operatorname{Lag}\left(L_{0}, M\right)$. Moreover, there exists a $\operatorname{Ham}(M)$ equivariant diffeomorphism $v: \operatorname{Lag}\left(L_{0}, M\right) / \operatorname{Diff}\left(L_{0}, \eta_{0}\right) \rightarrow \operatorname{Lag}_{p w}(M)$.

Proof. Define

$$
v: \operatorname{Lag}\left(L_{0}, M\right) / \operatorname{Diff}\left(L_{0}, \eta_{0}\right) \rightarrow \operatorname{Lag}_{p w}(M), \quad[i] \mapsto\left[\left(i, \eta_{0}\right)\right] .
$$

Then $v$ is injective since

$$
\left[\left(i_{1}, \eta_{0}\right)\right]=\left[\left(i_{2}, \eta_{0}\right)\right] \Rightarrow i_{2}=i_{1} \circ a^{-1}, \quad\left(a^{-1}\right)^{*} \eta_{0}=\eta_{0}
$$

for some $a \in \operatorname{Diff}_{+}\left(L_{0}\right)$. To check surjectivity suppose that $(L, \rho)=[(i, \eta)] \in \operatorname{Lag}_{p w}(M)$ is a positive weighted Lagrangian submanifold. By Moser's Lemma, since $\eta$ and $\eta_{0}$ are volume forms on $L_{0}$ that both induce the orientation of $L_{0}$ and integrate to 1 , there exists an isotopy $\psi_{t} \in \operatorname{Diff}_{+}\left(L_{0}\right)$ such that $\psi_{1}^{*} \eta_{0}=\eta$. Thus

$$
v\left(\left[i \circ \psi_{1}^{-1}\right]\right)=\left[\left(i \circ \psi_{1}^{-1}, \eta_{0}\right)\right]=[(i, \eta)] .
$$

We will now describe charts into spaces $\Gamma_{\text {closed }}\left(i^{*} T M\right) / \mathfrak{X}\left(L_{0}, \eta_{0}\right)$. Let $i \in \operatorname{Lag}\left(L_{0}, M\right)$ and let $\lambda_{i}: T^{*} L_{0} \supset W_{i} \rightarrow M$ be the symplectic embedding chosen in defining the chart $\left(U_{i}, \varphi_{i}\right)$ on 
$\operatorname{Lag}\left(L_{0}, M\right)$. Given a representative $X$ of a class $[X] \in \Gamma_{\text {closed }}\left(i^{*} T M\right) / \mathfrak{X}\left(L_{0}, \eta_{0}\right)$, the section $\mathrm{d} \lambda_{i}^{-1} \circ X \in \Gamma\left(\left.T\left(T^{*} L_{0}\right)\right|_{L_{0}}\right)$ can be decomposed as $\mathrm{d} \lambda_{i}^{-1} \circ X=\left.Z_{\alpha_{X}}\right|_{L_{0}}+Y$ where $\imath\left(Z_{\alpha_{X}}\right) \omega_{T^{*} L_{0}}=$ $\pi^{*} \alpha_{X}$ and $Y \in \mathfrak{X}\left(L_{0}\right)$. For a different choice of representative, this decomposition changes only in the component $Y$ tangent to $L_{0}$. Thus this decomposition defines a vector space isomorphism

$$
\zeta_{1}: \Gamma_{\text {closed }}\left(i^{*} T M\right) / \mathfrak{X}\left(L_{0}, \eta_{0}\right) \rightarrow Z^{1}\left(L_{0}\right) \oplus\left(\mathfrak{X}\left(L_{0}\right) / \mathfrak{X}\left(L_{0}, \eta_{0}\right)\right), \quad[X] \mapsto\left(\alpha_{X},[Y]\right),
$$

where $\mathrm{d} \lambda_{i}^{-1} \circ X=\left.Z_{\alpha_{X}}\right|_{L_{0}}+Y$.

Consider the map

$$
\zeta_{2}: \mathfrak{X}\left(L_{0}\right) / \mathfrak{X}\left(L_{0}, \eta_{0}\right) \rightarrow \Omega_{0}^{n}\left(L_{0}\right), \quad[Y] \mapsto \mathcal{L}_{Y} \eta_{0} .
$$

We claim that $\zeta_{2}$ is a vector space isomorphism. It is injective since $\mathcal{L}_{Y_{1}} \eta_{0}=\mathcal{L}_{Y_{2}} \eta_{0}$ implies that $Y_{1}-Y_{2} \in \mathfrak{X}\left(L_{0}, \eta_{0}\right)$. To check surjectivity, choose a metric $g_{0}$ on $L_{0}$ such that the induced volume form $\mu\left(g_{0}\right)$ equals $\eta_{0}$. Suppose that $\vartheta=\vartheta^{\prime} \cdot \eta_{0} \in \Omega_{0}^{n}\left(L_{0}\right)$ for $\vartheta^{\prime} \in C^{\infty}\left(L_{0}, \mathbb{R}\right)$. By the Hodge Decomposition Theorem (see e.g. [20]), there exists a function $h^{\prime} \in C^{\infty}\left(L_{0}, \mathbb{R}\right.$ ) (unique up to constants) such that $\triangle h^{\prime}=\vartheta^{\prime}$. For such an $h^{\prime}$, it follows that $\mathcal{L}_{\nabla h^{\prime}} \eta_{0}=\Delta h^{\prime} \cdot \eta_{0}=\vartheta$.

The isomorphisms $\zeta_{1}$ and $\zeta_{2}$ combine to define a vector space isomorphism $\zeta$ from the quotient $\Gamma_{\text {closed }}\left(i^{*} T M\right) / \mathfrak{X}\left(L_{0}, \eta_{0}\right)$ to $Z^{1}\left(L_{0}\right) \oplus \Omega_{0}^{n}\left(L_{0}\right)$. Let $E_{U_{[i]}}$ denote $\Gamma_{\text {closed }}\left(i^{*} T M\right) / \mathfrak{X}\left(L_{0}, \eta_{0}\right)$ equipped with the convenient structure induced by this isomorphism. Define

$$
\begin{aligned}
& U_{[i]}:=\left\{[l] \in \operatorname{Lag}\left(L_{0}, M\right) / \mathfrak{X}\left(L_{0}, \eta_{0}\right) \mid\left[\left(l, \eta_{0}\right)\right]=(N, \sigma) \in U_{\left[\left(i, \eta_{0}\right)\right]}\right. \\
& \left.\quad N=\lambda_{(i, \eta)}(\operatorname{Graph}(\alpha)), \alpha \in Z^{1}\left(L_{0}\right), \sigma=\left.\lambda_{(i, \eta)}^{-1}\right|_{N} ^{*} \pi^{*}\left(i^{*} \rho+\vartheta\right), \vartheta \in \Omega_{0}^{n}\left(L_{0}\right)\right\}, \\
& \varphi_{[i]}: U_{[i]} \rightarrow E_{U_{[i]}}, \quad \varphi_{[i]}([l]):=\zeta^{-1}(\alpha, \vartheta),
\end{aligned}
$$

where $\left(U_{\left[\left(i, \eta_{0}\right)\right]}, \varphi_{\left[\left(i, \eta_{0}\right)\right]}\right)$ is one of the charts on $\operatorname{Lag}_{p w}(M)$. The charts $\left(U_{[i]}, \varphi_{[i]}\right)$ indexed by $i \in \operatorname{Lag}\left(L_{0}, M\right)$ define a smooth atlas on $\operatorname{Lag}\left(L_{0}, M\right) / \mathfrak{X}\left(L_{0}, \eta_{0}\right)$.

From the definition of the smooth structure on $\operatorname{Lag}\left(L_{0}, M\right) / \mathfrak{X}\left(L_{0}, \eta_{0}\right)$, it follows that the map $v$ is a diffeomorphism. Since the $\operatorname{Ham}(M)$ action commutes with the $\operatorname{Diff}\left(L_{0}, \eta_{0}\right)$ action on $\operatorname{Lag}\left(L_{0}, M\right)$ and with the $\operatorname{Diff}_{+}\left(L_{0}\right)$ action on $\operatorname{Lag}_{p w}\left(L_{0}, M\right)$, we have well defined Ham $(M)$ actions on the quotients. Thus for $\phi \in \operatorname{Ham}(M)$,

$$
v(\phi \cdot[i])=v([\phi \circ i])=\left[\left(\phi \circ i, \eta_{0}\right)\right]=\phi \cdot\left[\left(i, \eta_{0}\right)\right]=\phi \cdot v([i])
$$

which verifies the $\operatorname{Ham}(M)$ equivariance of $v$.

As in the case of not necessarily positive weighted Lagrangian submanifolds, the smooth manifold $\operatorname{Lag}_{p w}\left(L_{0}, M\right)^{\mathcal{E}_{p w}}$ is the total space of a $\operatorname{Diff}_{+}\left(L_{0}\right)$ bundle over $\operatorname{Lag}_{p w}(M)^{\mathcal{F}_{p w}}$. We can define a basic 2 -form $\Omega$ on $\operatorname{Lag}_{p w}\left(L_{0}, M\right)^{\mathcal{E}_{p w}}$ by the expression

$$
\Omega_{(i, \eta)}\left(\left(X_{1}, \vartheta_{1}\right),\left(X_{2}, \vartheta_{2}\right)\right):=\int_{L_{0}}\left[\omega\left(X_{1}, X_{2}\right) \eta+h_{1} \vartheta_{2}-h_{2} \vartheta_{1}\right]
$$

where $\alpha_{X_{k}}=d h_{k}$ for $h_{k} \in C^{\infty}\left(L_{0}, \mathbb{R}\right)$. This then descends to a weak symplectic structure (also labeled $\Omega$ ) on $\operatorname{Lag}_{p w}(M)^{\mathcal{F}_{p w}}$. So in particular the isodrasts in $\operatorname{Lag}_{p w}(M)$ are weakly symplectic manifolds.

\section{Embeddings into a symplectic manifold}

In this section we will make precise a heuristic construction by Donaldson [3] of a symplectic structure and moment map for a diffeomorphism group action restricted to the space of embeddings. 
Let $S_{0}$ be a fixed finite dimensional, compact, and oriented manifold equipped with a volume form $\eta_{0}$, and let $(M, \omega)$ be a finite dimensional symplectic manifold. The set of embeddings $\operatorname{Emb}\left(S_{0}, M\right)$ of $S_{0}$ into $M$ is an open subset of the space $C^{\infty}\left(S_{0}, M\right)$ of all smooth maps. Thus $\operatorname{Emb}\left(S_{0}, M\right)$ is a smooth manifold modeled on spaces $\Gamma\left(i^{*} T M\right)$ for $i \in \operatorname{Emb}\left(S_{0}, M\right)$. Assign to each point $i \in \operatorname{Emb}\left(S_{0}, M\right)$ a skew symmetric bilinear form on $T_{i} \operatorname{Emb}\left(S_{0}, M\right)$ via the expression

$$
\Omega_{i}^{D}\left(X_{1}, X_{2}\right):=\int_{S_{0}} \omega\left(X_{1}, X_{2}\right) \eta_{0}
$$

for $X_{1}, X_{2} \in T_{i} \operatorname{Emb}\left(S_{0}, M\right)$.

Proposition 12. The pointwise assignment $\Omega^{D}$ in (7) defines a weak symplectic structure on $\operatorname{Emb}\left(S_{0}, M\right)$.

Proof. Checking smoothness amounts to checking the following statement: If $X_{1}(t)$ and $X_{2}(t)$ are smooth time dependent vector fields on $M, i_{t}$ is a smooth curve in $\operatorname{Emb}\left(S_{0}, M\right)$, and $s: \mathbb{R} \rightarrow \mathbb{R}$ is a smooth function, then the map

$$
\begin{aligned}
\mathbb{R} & \rightarrow \mathbb{R} \\
t & \mapsto \int_{S_{0}} \omega_{i_{s(t)}}\left(X_{1}(t) \circ i_{s(t)}, X_{2}(t) \circ i_{s(t)}\right) \eta_{0}
\end{aligned}
$$

is smooth. This statement follows from the smoothness of all functions in the integrand.

We will now prove closedness by choosing special extensions of tangent vectors to vector fields on $\operatorname{Emb}\left(S_{0}, M\right)$. Let $X_{1}, X_{2}, X_{3} \in T_{i} \operatorname{Emb}\left(S_{0}, M\right)$ be tangent vectors. Let $Z_{1}, Z_{2}$ and $Z_{3}$ be vector fields defined on a neighbourhood of $i\left(S_{0}\right)$ in $M$ such that $Z_{k} \circ i=X_{k}$. Let $\xi_{1}, \xi_{2}$ and $\xi_{3}$ be vector fields defined on the chart neighbourhood $U_{i} \subset \operatorname{Emb}\left(S_{0}, M\right)$ by the expression $\xi_{k}(j):=Z_{k} \circ j$. For these particular vector fields, Lie brackets like $\left[\xi_{1}, \xi_{2}\right]$ at a point $i \in$ $\operatorname{Emb}\left(S_{0}, M\right)$ can be written in terms of the Lie bracket $\left[Z_{1}, Z_{2}\right]$ :

$$
\left[\xi_{1}, \xi_{2}\right](i)=\mathrm{d} \xi_{2}(i) \cdot \xi_{1}(i)-\mathrm{d} \xi_{1}(i) \cdot \xi_{2}(i)=\mathrm{d} Z_{2} \cdot Z_{1} \circ i-\mathrm{d} Z_{1} \cdot Z_{2} \circ i=\left[Z_{1}, Z_{2}\right] \circ i .
$$

Thus,

$$
\begin{aligned}
d \Omega_{i}^{D}\left(X_{1}, X_{2}, X_{3}\right)= & \left(d \Omega^{D}\left(\xi_{1}, \xi_{2}, \xi_{3}\right)\right)_{i}=\left(\xi_{1} \Omega^{D}\left(\xi_{2}, \xi_{3}\right)+\xi_{2} \Omega^{D}\left(\xi_{3}, \xi_{1}\right)+\xi_{3} \Omega^{D}\left(\xi_{1}, \xi_{2}\right)\right. \\
& \left.-\Omega^{D}\left(\left[\xi_{1}, \xi_{2}\right], \xi_{3}\right)-\Omega^{D}\left(\left[\xi_{2}, \xi_{3}\right], \xi_{1}\right)-\Omega^{D}\left(\left[\xi_{3}, \xi_{2}\right], \xi_{1}\right)\right)_{i} \\
= & \int_{S_{0}} d \omega\left(Z_{1}, Z_{2}, Z_{3}\right) \eta_{0}=0
\end{aligned}
$$

by closedness of $\omega$.

As for weak nondegeneracy, suppose that $X_{1} \in \Gamma\left(i^{*} T M\right)$ is nonzero on a neighbourhood $W$ of $x \in S_{0}$. Let $j$ be a compatible almost complex structure on $M$ (i.e. $g(\cdot, \cdot):=\omega(\cdot, j \cdot)$ is a Riemannian metric on $M)$. Let $\chi$ be a positive function supported on $W$. Define $X_{2} \in$ $\Gamma\left(i^{*} T M\right)$ by $X_{2}(x):=\chi(i(x)) \cdot j X_{1}(x)$. It follows that

$$
\int_{S_{0}} \omega\left(X_{1}, X_{2}\right) \eta_{0}=\int_{S_{0}}(\chi \circ i) \cdot g\left(X_{1}, X_{1}\right) \eta_{0}>0
$$

Remark 11. Donaldson originally defined the above weakly symplectic structure $\Omega^{D}$ on the space of smooth mappings $C^{\infty}\left(S_{0}, M\right)$. When $S_{0}=M$, the 2 -form $\Omega^{D}$ restricts to a symplectic structure defined by Khesin and Lee on the open subset of orientation preserving diffeomorphisms of $M$ (relative to the orientation induced by the symplectic volume form, which is taken to be $\eta_{0}$; see Section 3 in [8]). 
The Lie group $\operatorname{Diff}\left(S_{0}, \eta_{0}\right)$ acts freely on $\operatorname{Emb}\left(S_{0}, M\right)$ via

$$
a \cdot i:=i \circ a^{-1} \text {. }
$$

Definition 8. Let $G$ be a Lie group with Lie algebra $\mathfrak{g}$. By $\mathfrak{g}^{*}$ we will mean all bounded linear functionals on the convenient vector space $\mathfrak{g}$. Let $\langle\cdot, \cdot\rangle: \mathfrak{g}^{*} \times \mathfrak{g} \rightarrow \mathbb{R}$ denote the canonical pairing between $\mathfrak{g}^{*}$ and $\mathfrak{g}$. The coadjoint representation of $G, \mathrm{Ad}^{*}: G \rightarrow G L\left(\mathfrak{g}^{*}\right) \subset L\left(\mathfrak{g}^{*}, \mathfrak{g}^{*}\right)$, is defined by

$$
\left\langle\operatorname{Ad}_{g}^{*} \zeta, \xi\right\rangle:=\left\langle\zeta, \operatorname{Ad}_{g^{-1}} \xi\right\rangle \quad \text { for any } \xi \in \mathfrak{g} .
$$

Definition 9. Let $(M, \sigma)$ be a weakly symplectic smooth manifold. Let $G \times M \rightarrow M$ be a smooth action of a Lie group $G$ on $M$, such that $l_{g}^{*} \sigma=\sigma$ for all $g \in G$. This $G$ action is called Hamiltonian if there exists a $G$ equivariant map (called the moment map) $\mu \in C^{\infty}\left(M, \mathfrak{g}^{*}\right.$ ) such that for all $\xi \in \mathfrak{g}$, the function $\langle\mu, \xi\rangle \in C^{\infty}(M, \mathbb{R})$ is a Hamiltonian for $\xi_{M}$ :

$$
\mathrm{d}\langle\mu, \xi\rangle=\imath\left(\xi_{M}\right) \sigma .
$$

Proposition 13. If $i^{*}[\omega]$ is the zero class in $H^{2}\left(S_{0}\right)$ for all $i \in \operatorname{Emb}\left(S_{0}, M\right)$ and $H^{1}\left(S_{0}\right)=0$, then the $\operatorname{Diff}\left(S_{0}, \eta_{0}\right)$ action on $\operatorname{Emb}\left(S_{0}, M\right)$ is Hamiltonian.

Proof. We first note that the $\operatorname{Diff}\left(S_{0}, \eta_{0}\right)$ action on $\operatorname{Emb}\left(S_{0}, M\right)$ is symplectic:

$$
\begin{aligned}
\left(l_{a}^{*} \Omega^{D}\right)_{i}\left(X_{1}, X_{2}\right) & =\int_{S_{0}} \omega_{i \circ a^{-1}(x)}\left(X_{1} \circ a^{-1}(x), X_{2} \circ a^{-1}(x)\right) \eta_{0}(x) \\
& =\int_{S_{0}} \omega_{i(y)}\left(X_{1}(y), X_{2}(y)\right)\left(a^{-1}\right)^{*} \eta_{0}(y)=\Omega_{i}^{D}\left(X_{1}, X_{2}\right) .
\end{aligned}
$$

If $i^{*} \omega=d A$, define $\mu: \operatorname{Emb}\left(S_{0}, M\right) \rightarrow \mathfrak{X}\left(S_{0}, \eta_{0}\right)^{*}$ by

$$
\langle\mu, \xi\rangle:=\int_{S_{0}} A(\xi) \eta_{0}
$$

This definition is independent of the choice of $A$ since $H^{1}\left(S_{0}\right)=0$. The map $\mu$ is smooth by the usual local arguments. To check that $\mu$ is a moment map, let $X \in \Gamma\left(i^{*} T M\right)$ be a tangent vector at $i \in \operatorname{Emb}\left(S_{0}, M\right)$. Let $Z$ be a vector field on a neighbourhood of $i\left(S_{0}\right)$ satisfying $Z \circ i=X$, and suppose $Z$ generates a flow $\tau_{t}$ on $M$. Let $A_{t}$ be a smooth curve in $\Omega^{1}\left(S_{0}\right)$ satisfying $d A_{t}:=\left(\tau_{t} \circ i\right)^{*} \omega$. Then

$$
\left.\frac{d}{d t}\right|_{t=0} d A_{t}=\left.i^{*} \frac{d}{d t}\right|_{t=0} \tau_{t}^{*} \omega=i^{*} \mathcal{L}_{Z} \omega=i^{*}(d \circ \imath(Z) \omega) .
$$

This together with $H^{1}\left(S_{0}\right)=0$ imply that $\left.\frac{d}{d t}\right|_{t=0} A_{t}=i^{*} \imath(Z) \omega+d h$ for some $h \in C^{\infty}\left(S_{0}, \mathbb{R}\right)$. It follows that

$$
\langle d \mu(X), \xi\rangle=\int_{S_{0}}\left[\omega\left(X, i_{*} \xi\right)+d h(\xi)\right] \eta_{0}=\Omega^{D}\left(X, i_{*} \xi\right)
$$

which verifies the moment map condition.

Finally, $\mu$ is $\operatorname{Diff}\left(S_{0}, \eta_{0}\right)$ equivariant:

$$
\begin{aligned}
\left\langle\mu\left(l_{a}(i)\right), \xi\right\rangle & =\int_{S_{0}}\left(a^{-1}\right)^{*} A(\xi) \eta_{0}=\int_{S_{0}} A\left(\mathrm{~d} a^{-1} \cdot \xi\right) \eta_{0} \\
& =\left\langle\mu(i), \operatorname{Ad}_{a^{-1}} \xi\right\rangle=\left\langle\operatorname{Ad}_{a}^{*} \mu(i), \xi\right\rangle .
\end{aligned}
$$


Let us now consider the special case when $\int_{S_{0}} \eta_{0}=1$, the manifold $S_{0}$ is half the dimension of $M$, and assume that the topological conditions $H^{1}\left(S_{0}\right)=0$ and $\left[i^{*} \omega\right]=0 \in H^{2}\left(S_{0}\right)$ in Proposition 13 hold so that we have a moment map $\mu$ on $\operatorname{Emb}\left(S_{0}, M\right)$. The level surface $\mu^{-1}\{0\}$ is given by

$$
\mu^{-1}\{0\}=\left\{i \in \operatorname{Emb}\left(S_{0}, M\right) \mid i^{*} \omega=0\right\}=\operatorname{Lag}\left(S_{0}, M\right) .
$$

The group $\operatorname{Diff}\left(S_{0}, \eta_{0}\right)$ acts freely on $\mu^{-1}\{0\}$ in the usual way, with the quotient given by

$$
\mu^{-1}\{0\} / \operatorname{Diff}\left(S_{0}, \eta_{0}\right)=\operatorname{Lag}\left(S_{0}, M\right) / \operatorname{Diff}\left(S_{0}, \eta_{0}\right) .
$$

By Proposition 11, the set $\operatorname{Lag}\left(S_{0}, M\right) / \operatorname{Diff}\left(S_{0}, \eta_{0}\right)$ is a smooth manifold modeled on convenient spaces $\Gamma_{\text {closed }}\left(i^{*} T M\right) / \mathfrak{X}\left(S_{0}, \eta_{0}\right)$ for $i \in \operatorname{Lag}\left(S_{0}, M\right)$. In fact, the manifold $\operatorname{Lag}\left(S_{0}, M\right)$ is the total space of a principal $\operatorname{Diff}\left(S_{0}, \eta_{0}\right)$ bundle over the quotient $\operatorname{Lag}\left(S_{0}, M\right) / \operatorname{Diff}\left(S_{0}, \eta_{0}\right)$. Since the 2 -form $\left.\Omega^{D}\right|_{\mu^{-1}\{0\}}$ is basic, it descends to a unique 2 -form $\Omega_{\text {red }}^{D}$ on $\mu^{-1}\{0\} / \operatorname{Diff}\left(S_{0}, \eta_{0}\right)$.

Under the topological assumption that $H^{1}\left(S_{0}\right)=0$, the subspace given by the isodrastic foliation $\mathcal{F}_{p w}$ at each point equals the entire tangent space to $\operatorname{Lag}_{p w}(M)$ at that point. The weak symplectic structure $\Omega$ on isodrasts in this case becomes well defined on all of $\operatorname{Lag}_{p w}(M)$. In fact, the pull back of $\Omega$ under the diffeomorphism $v$ in Proposition 11 is given by

$$
\left(v^{*} \Omega\right)\left(\left[X_{1}\right],\left[X_{2}\right]\right)=\int_{L_{0}} \omega\left(X_{1}, X_{2}\right) \eta_{0}=\Omega_{\mathrm{red}}^{D}\left(\left[X_{1}\right],\left[X_{2}\right]\right) .
$$

In other words, the "symplectic quotient" $\left(\mu^{-1}\{0\} / \operatorname{Diff}_{+}\left(S_{0}, \eta_{0}\right), \Omega_{\text {red }}^{D}\right)$ is symplectomorphic to $\left(\operatorname{Lag}_{p w}(M), \Omega\right)$.

This last result is suggestive, leading one to wonder if the symplectic structure $\Omega^{D}$ on $\operatorname{Emb}\left(S_{0}, M\right)$ might be related to the symplectic structure $\Omega$ on isodrasts in $\operatorname{Lag}_{p w}(M)$ via some sort of reduction procedure. In the next section we will make this relationship clear.

\section{$7 \quad$ Reduction of $\left(\operatorname{Emb}\left(S_{0}, M\right), \Omega^{D}\right)$}

In this section we will define a notion of reduction of weakly symplectic spaces with respect to a group action. We will then show that the image of $\operatorname{Ham}(M)$ orbits through isotropic embeddings in $\operatorname{Emb}\left(S_{0}, M\right)$ under the projection to the quotient $\operatorname{Emb}\left(S_{0}, M\right) / \operatorname{Diff}\left(S_{0}, \eta_{0}\right)$ are reductions. Moreover, these reductions are naturally symplectomorphic to the spaces of positive weighted isotropic submanifolds of $M$. In particular, when $\operatorname{dim} S_{0}=\frac{1}{2} \operatorname{dim} M$, the particular reductions of $\mathrm{Emb}\left(S_{0}, M\right)$ consisting of positive weighted Lagrangian submanifolds are symplectomorphic to isodrasts in $\operatorname{Lag}_{p w}(M)$.

In Proposition 13, the topological assumption $H^{1}\left(S_{0}\right)=0$ was essential in defining a moment map. Since the transverse spaces to the leaves of an isodrastic foliation become nontrivial exactly when $H^{1}\left(L_{0}\right)$ is nontrivial, we would like to remove such a topological condition on $S_{0}$. This means we must use a notion of reduction that does not depend on having a moment map.

Let us begin by looking at the standard reduction of a finite dimensional symplectic manifold $(P, \sigma)$ with respect to a Hamiltonian $G$ action using a moment map $\mu$. Suppose $r$ is a regular value of $\mu$. The tangent space at $p$ to the level surface $\mu^{-1}\{r\}$ is equal to the set $\mathcal{D}_{p}$ of all vectors $X \in T_{p} P$ satisfying $\sigma\left(X, \xi_{P}(p)\right)=0$ for all $\xi \in \mathfrak{g}$. These subspaces $\mathcal{D}_{p}$ are defined for any symplectic action on $P$, even in the absence of a moment map, and define a distribution $\mathcal{D}$ on $P$. If $G \circlearrowright P$ is a free symplectic action, then this distribution can be taken as the starting point of the "optimal reduction method" of Juan-Pablo Ortega and Tudor S. Ratiu [11]. We will not describe the details here, but simply note that for a symplectic $G$ action $G \circlearrowright(P, \sigma)$ 
- the optimal reduction method yields symplectic spaces $\left(P_{\rho}, \sigma_{\rho}\right)$ where $\rho \in P / G_{\mathcal{D}}$ and $G_{\mathcal{D}}$ is the pseudogroup of flows generated by Hamiltonian vector fields in $\mathfrak{X}_{\mathcal{D}}(P)$ corresponding to $G$-invariant Hamiltonian functions;

- the "optimal momentum map" is given by the projection $\mathcal{J}: P \rightarrow P / G_{\mathcal{D}}$;

- each reduced symplectic space $\left(P_{\rho}, \sigma_{\rho}\right)$ is the quotient of an integral manifold of $\mathcal{D}$ (the level surface $\mathcal{J}^{-1}\{\rho\}$ ) by the stabilizer $G_{\rho}$ at $\rho$ under the action $G \circlearrowright P / G_{\mathcal{D}}: g \cdot[p]:=[g \cdot p]$;

- if $G \circlearrowright P$ is a free Hamiltonian action with moment map $\mu$, and the point $r \in \mathfrak{g}^{*}$ is a regular value of $\mu$, and $\mu^{-1}\{r\}$ is connected, then $\mu^{-1}\{r\}$ is a $G_{\mathcal{D}}$ orbit $\rho$ and the reduced symplectic space $P_{\rho}$ coincides with the symplectic quotient $\mu^{-1}\{r\} / G_{r}$ (here $G_{r}$ denotes the stabilizer of $r \in \mathfrak{g}^{*}$ with respect to the coadjoint action of $G$ ).

This suggests a way to define reduction in the infinite dimensional case, and motivates the following definition:

Definition 10. Let $(P, \sigma)$ be a weakly symplectic smooth manifold. Let $G \circlearrowright P$ be a smooth free action of a Lie group $G$ on $P$, such that $l_{g}^{*} \sigma=\sigma$ for all $g \in G$. The collection of subspaces

$$
\mathcal{D}_{x}:=\left\{X \in T_{x} M \mid \sigma_{x}\left(X, \xi_{M}(x)\right)=0 \forall \xi \in \mathfrak{g}\right\}
$$

for $x \in P$ defines a distribution $\mathcal{D}$ on $P$. Let $i_{N}: N \hookrightarrow P$ be a maximal integral manifold of $\mathcal{D}$ and let $q: P \rightarrow P / G$ denote the projection to the orbit space. Suppose that

1) $q(N)$ is a smooth manifold,

2) there exists a unique weak symplectic structure $\sigma_{\text {red }}$ on $q(N)$ such that $\left(\left.q\right|_{N}\right)^{*} \sigma_{\text {red }}=i_{N}^{*} \sigma$.

Then the weakly symplectic manifold $\left(q(N), \sigma_{\text {red }}\right)$ will be called a reduction or symplectic quotient of $(P, \sigma)$ with respect to the $G$ action.

\section{Convention}

From now on, we will make no topological assumptions on $i^{*} \omega$ or $H^{1}\left(S_{0}\right)$.

For the $\operatorname{Diff}\left(S_{0}, \eta_{0}\right)$ action on the symplectic manifold $\left(\operatorname{Emb}\left(S_{0}, M\right), \Omega^{D}\right)$, the subspaces $\mathcal{D}_{i}$ can be described in very familiar terms:

Proposition 14. For every $i \in \operatorname{Emb}\left(S_{0}, M\right)$,

$$
\mathcal{D}_{i}=\Gamma_{\text {exact }}\left(i^{*} T M\right)=\left\{X \in \Gamma\left(i^{*} T M\right) \mid \alpha_{X} \in B^{1}\left(S_{0}\right)\right\} .
$$

Proof. The distribution $\mathcal{D}$ on $\operatorname{Emb}\left(S_{0}, M\right)$ is defined by

$$
\mathcal{D}_{i}:=\left\{X \in \Gamma\left(i^{*} T M\right) \mid \Omega_{i}^{D}\left(X, \xi_{\operatorname{Emb}\left(S_{0}, M\right)}(i)\right)=0, \forall \xi \in \mathfrak{X}\left(S_{0}, \eta_{0}\right)\right\}
$$

for $i \in \operatorname{Emb}\left(S_{0}, M\right)$. Since $\int_{S_{0}} d h(\xi) \eta_{0}=0$ for any function $h$ on $S_{0}$ and all $\xi \in \mathfrak{X}\left(S_{0}, \eta_{0}\right)$, it follows that $\left\{X \in \Gamma\left(i^{*} T M\right) \mid \alpha_{X} \in B^{1}\left(S_{0}\right)\right\} \subset \mathcal{D}_{i}$.

Let $X \in \mathcal{D}_{i}$, i.e. $\int_{S_{0}} \alpha_{X}(\xi) \eta_{0}=\int_{S_{0}} \alpha_{X} \wedge \imath_{\xi} \eta_{0}=0$ for all divergence free $\xi$. If $U$ is a coordinate neighbourhood in $S_{0}, \eta_{0}=\eta d x_{1} \wedge \cdots \wedge d x_{n}$ on $U$, and $f$ a function with supp $(f) \subset U$, then define the divergence free vector field $Y_{12}:=\frac{1}{\eta}\left(\frac{\partial f}{\partial x_{2}} \frac{\partial}{\partial x_{1}}-\frac{\partial f}{\partial x_{1}} \frac{\partial}{\partial x_{2}}\right)$. If $\alpha_{X}=a_{i} d x_{i}$ on $U$, then

$$
\begin{aligned}
\int_{S_{0}} \alpha_{X} \wedge \imath_{Y_{12}} \eta_{0} & =\int_{U}\left(a_{1} \frac{\partial f}{\partial x_{2}}-a_{2} \frac{\partial f}{\partial x_{1}}\right) d x_{1} \wedge \cdots \wedge d x_{n} \\
& =\int_{U}\left(\frac{\partial a_{2}}{\partial x_{1}}-\frac{\partial a_{1}}{\partial x_{2}}\right) f d x_{1} \wedge \cdots \wedge d x_{n}
\end{aligned}
$$


where we have used integration by parts. If this is to vanish for all $f$ then $\frac{\partial a_{2}}{\partial x_{1}}=\frac{\partial a_{1}}{\partial x_{2}}$. Similarly, by considering vector fields like $Y_{13}:=\frac{1}{\eta}\left(\frac{\partial f}{\partial x_{3}} \frac{\partial}{\partial x_{1}}-\frac{\partial f}{\partial x_{1}} \frac{\partial}{\partial x_{3}}\right)$ it follows that $\frac{\partial a_{3}}{\partial x_{1}}=\frac{\partial a_{1}}{\partial x_{3}}$, etc., which means $\alpha_{X}$ is closed.

Let $g_{0}$ be a Riemannian metric on $S_{0}$ whose volume form equals $\eta_{0}$. For every $X \in \mathcal{D}_{i}$, since $\alpha_{X}$ is closed, there exists a function $h$ on $S_{0}$ such that $\beta_{X}:=\alpha_{X}-d h$ is harmonic. Moreover, $\int_{S_{0}} \beta_{X}(\xi) \eta_{0}=0$ for every $\xi \in \mathfrak{X}\left(S_{0}, \eta_{0}\right)$. Define the vector field $Y_{\beta_{X}}$ on $S_{0}$ by $\beta_{X}=g_{0}\left(Y_{\beta_{X}}, \cdot\right)$. Let $V$ be a coordinate neighbourhood in $S_{0}$, and suppose $\beta_{X}=b_{i} d x_{i}$ on $V$. On $V$,

$$
\begin{aligned}
\mathcal{L}_{Y_{\beta_{X}}} \eta_{0} & =d \circ \imath_{Y_{\beta_{X}}}\left(\sqrt{\operatorname{det} g_{0}} d x_{1} \wedge \cdots \wedge d x_{n}\right) \\
& =d\left(\sqrt{\operatorname{det} g_{0}} g_{0}^{i j} b_{j}(-1)^{i} d x_{1} \wedge \cdots \wedge \hat{d x_{i}} \wedge \cdots \wedge d x_{n}\right)=d * \beta_{X}=* \delta \beta_{X}=0
\end{aligned}
$$

which means $Y_{\beta_{X}} \in \mathfrak{X}\left(S_{0}, \eta_{0}\right)$. So $0=\int_{S_{0}} \beta_{X}\left(Y_{\beta_{X}}\right) \eta_{0}=\int_{S_{0}} g_{0}\left(Y_{\beta_{X}}, Y_{\beta_{X}}\right) \eta_{0}$ implies that $\beta_{X}=0$, which means $\alpha_{X}=d h$.

The group $\operatorname{Ham}(M)$ acts freely on $\operatorname{Emb}\left(S_{0}, M\right)$ under the action

$$
\operatorname{Ham}(M) \circlearrowright \operatorname{Emb}\left(S_{0}, M\right): \quad \phi \cdot i:=\phi \circ i .
$$

In what follows we will show that $\operatorname{Ham}(M)$ orbits through isotropic embeddings are maximal integral manifolds of $\mathcal{D}$. For this purpose, we will need to make use of the following isotropic embedding theorem of Weinstein:

Theorem 3 (see [22]). Let $(M, \omega)$ be a symplectic manifold and $i: I \hookrightarrow M$ be an isotropic submanifold, i.e. $i^{*} \omega=0$. The vector bundle $T^{*} I \oplus\left(T I^{\omega} / T I\right)$ admits a symplectic structure on a neighbourhood of the zero section, which is given by $\omega_{T^{*} I}+\omega_{\mathbb{R}^{2 n}}$ on the zero section. Furthermore, there exists a neighbourhood $U_{1}$ of $I$ in $M$, a neighbourhood $U_{2}$ of $I$ in $T^{*} I \oplus$ $\left(T I^{\omega} / T I\right)$, and a symplectomorphism from $U_{1}$ to $U_{2}$ fixing $I$.

Proposition 15. If $i \in \operatorname{Emb}\left(S_{0}, M\right)$ is an isotropic embedding, then the $\operatorname{Ham}(M)$ orbit $\mathcal{N}$ through $i$ is a maximal integral manifold of the distribution $\mathcal{D}\left(\right.$ see (8)). The restriction of $\Omega^{D}$ to $\mathcal{N}$ in $\operatorname{Emb}\left(S_{0}, M\right)$ descends to a unique weak symplectic structure $\Omega_{\text {red }}^{D}$ on the image $\mathcal{O}:=q(\mathcal{N})$ in the orbit space under the projection $q: \operatorname{Emb}\left(S_{0}, M\right) \rightarrow \operatorname{Emb}\left(S_{0}, M\right) / \operatorname{Diff}\left(S_{0}, \eta_{0}\right)$. Thus the symplectic spaces $\left(\mathcal{O}, \Omega_{\mathrm{red}}^{D}\right)$ are reductions of $\operatorname{Emb}\left(S_{0}, M\right)$ with respect to the $\operatorname{Diff}\left(S_{0}, \eta_{0}\right)$ action.

Proof. We will first show that a Ham $(M)$ orbit through an isotropic embedding is an integral manifold of $\mathcal{D}$. Suppose that $i: S_{0} \rightarrow M$ is an isotropic embedding. By Theorem 3, if $S=$ $i\left(S_{0}\right)$ and $N=T S^{\omega} / T S$ denotes the symplectic normal bundle, then by choosing a symplectic embedding $\lambda: M \supset U \rightarrow T^{*} S \oplus N$ we can assume that $M=T^{*} S \oplus N$ and that $i$ is the zero section inclusion. Let $\operatorname{pr}_{1}: T^{*} S \oplus N \rightarrow T^{*} S$ and $\mathrm{pr}_{2}: T^{*} S \oplus N \rightarrow N$ be the natural maps which forget the points in the symplectic normal and cotangent fibers respectively. That is, $\operatorname{pr}_{1}(x, p, v):=(x, p)$ and $\operatorname{pr}_{2}(x, p, v):=(x, v)$. Given $X \in \mathcal{D}_{i}$ with $\alpha_{X}=d h$ for some $h \in C^{\infty}\left(S_{0}, \mathbb{R}\right)$, let $X=X_{\text {fib }}+X_{\text {tan }}$ denote the decomposition of $X$ into components tangent to the fibers and tangent to $S$. Extend $X_{\text {fib }}$ constantly along the fibers in $T^{*} S \oplus N$ to a vector field $Z$ defined on a neighbourhood of the zero section. It follows that $Z$ is a Hamiltonian vector field satisfying $\imath(Z) \omega=d\left(\operatorname{pr}_{1}^{*} \pi_{T^{*} S}^{*} f+\operatorname{pr}_{2}^{*} H_{N}\right)$ where $f \in C^{\infty}(S, \mathbb{R})$ satisfies $i^{*} f=h$, and $H_{N} \in C^{\infty}(N, \mathbb{R})$ is defined by $H_{N}(x, v):=\omega_{\mathbb{R}^{2 n}}\left(\operatorname{dpr}_{2} \circ X(x), v\right)$. Since $\omega=\omega_{T^{*} S}+\omega_{\mathbb{R}^{2 n}}$ along the zero section, $\left.\imath\left(X_{\tan }\right) \omega\right|_{T S}=0$. Thus for each point $x$ in the zero section, we have that $\imath\left(X_{\tan }\right) \omega$ defines a linear functional $\left(\imath\left(X_{\tan }\right) \omega\right)_{x}$ on the fiber $T_{x}^{*} S \oplus N_{x}$. The smooth function $H_{\tan }: T^{*} S \oplus N \rightarrow \mathbb{R}$ defined by $H_{\tan }(x, p, v):=\left(\imath\left(X_{\tan }\right) \omega\right)_{x}(p, v)$ is the primitive of a Hamiltonian vector field $Z_{\text {tan }}$ satisfying $Z_{\tan } \circ i=X_{\text {tan }}$. It follows that $\mathcal{D}_{i} \subset T_{i}(\operatorname{Ham}(M) \cdot i)$. The converse inclusion follows from the fact that $\alpha_{X_{H} \circ i}=d i^{*} H$ for any Hamiltonian vector field 
$X_{H}$. So Ham $(M)$ orbits must be integral manifolds of $\mathcal{D}$. The fact that they must be maximal can be shown as in the proof of Proposition 4.

Let $\mathcal{N}$ be a $\operatorname{Ham}(M)$ orbit through an isotropic embedding $i$. The image $\mathcal{O}:=q(\mathcal{N})$ is equal to the $\operatorname{Ham}(M)$ orbit in the quotient $\operatorname{Emb}\left(S_{0}, M\right) / \operatorname{Diff}\left(S_{0}, \eta_{0}\right)$ under the action $\phi \cdot[i]:=[\phi \circ i]$, it consists of positive weighted isotropic submanifolds of $M$ diffeomorphic to $S_{0}$, and can be given a smooth manifold structure similar to that on the space of positive weighted Lagrangian submanifolds. The space $\operatorname{Diff}\left(S_{0}, \eta_{0}\right) \cdot \mathcal{N}$ is the total space of a principal $\operatorname{Diff}\left(S_{0}, \eta_{0}\right)$ bundle over $\mathcal{O}$. The pullback of $\Omega^{D}$ to $\operatorname{Diff}\left(S_{0}, \eta_{0}\right) \cdot \mathcal{N}$ defines a closed basic 2 -form which descends to a closed 2 -form $\Omega_{\text {red }}^{D}$ on $\mathcal{O}$. To check weak nondegeneracy, we first note that the 2 -form $\Omega_{\text {red }}^{D}$ is given by the expression

$$
\Omega_{\mathrm{red}}^{D}\left(\left[X_{1}\right],\left[X_{2}\right]\right)=\int_{S_{0}} \omega\left(X_{1}, X_{2}\right) \eta_{0}
$$

Again, by choosing a symplectic embedding $\lambda: M \supset U \rightarrow T^{*} S \oplus N$ we can assume that $M=$ $T^{*} S \oplus N$ and that $i$ is the zero section inclusion. Given $\left[X_{2}\right] \in \operatorname{Ker}\left(\Omega_{\text {red }}^{D}\right)$, let $X_{2}=X_{\text {fib }}+X_{\tan }$ be the decomposition of $X_{2}$ into components tangent to the fibers and to the zero section respectively. Extend $X_{\text {fib }}$ and $X_{\text {tan }}$ to Hamiltonian vector fields $Z_{\text {fib }}$ and $Z_{\text {tan }}$ respectively as before. For $\left[X_{1}\right] \in T_{[i]} \mathcal{O}$, let $Z_{f_{1}}$ be a Hamiltonian vector field on $T^{*} S \oplus N$ such that $X_{1}=Z_{f_{1}} \circ i$. It follows that

$$
\begin{aligned}
0=\Omega_{\mathrm{red}}^{D}\left(\left[X_{1}\right],\left[X_{2}\right]\right) & =\int_{S_{0}} \omega\left(Z_{f_{1}}, X_{2}\right) \eta_{0}=\int_{S_{0}} i^{*}\left[\omega\left(Z_{f_{1}}, Z_{\mathrm{fib}}\right)\right] \eta_{0}+\int_{S_{0}} i^{*}\left[\omega\left(Z_{f_{1}}, Z_{\mathrm{tan}}\right)\right] \eta_{0} \\
& =\int_{S_{0}} i^{*} \mathcal{L}_{Z_{\mathrm{fib}}} f_{1} \eta_{0}+\int_{S_{0}} i^{*} \mathcal{L}_{Z_{\mathrm{tan}}} f_{1} \eta_{0}=\int_{S_{0}} i^{*} \mathcal{L}_{Z_{\mathrm{fib}}} f_{1} \eta_{0}+\int_{S_{0}} \mathcal{L}_{Y}\left(i^{*} f_{1}\right) \eta_{0},
\end{aligned}
$$

where $Y \in \mathfrak{X}\left(S_{0}\right)$ and $i_{*} Y=Z_{\tan } \circ i$. If $f_{1}$ is the pullback through the bundle projection of a function on the base $S$, then the first term vanishes and the second term vanishes if and only if $Y \in \mathfrak{X}\left(S_{0}, \eta_{0}\right)$. It follows that $Z_{\text {fib }}$ must vanish which means $\left[X_{2}\right]=0$.

Finally, uniqueness of the 2 -form $\Omega_{\text {red }}^{D}$ follows from the fact that the principal $\operatorname{Diff}\left(S_{0}, \eta_{0}\right)$ bundle $\operatorname{Emb}\left(S_{0}, \eta_{0}\right) \rightarrow \operatorname{Emb}\left(S_{0}, \eta_{0}\right) / \operatorname{Diff}\left(S_{0}, \eta_{0}\right)$ restricted to $\mathcal{O}$ admits local sections.

Thus the image $\mathcal{O}:=q(\mathcal{N})$ of a $\operatorname{Ham}(M)$ orbit in $\operatorname{Emb}\left(S_{0}, M\right)$ through an isotropic embedding is a symplectic quotient of $\left(\operatorname{Emb}\left(S_{0}, M\right), \Omega^{D}\right)$ with respect to the $\operatorname{Diff}\left(S_{0}, M\right)$ action.

\section{Convention}

From now on we will assume that $\int_{S_{0}} \eta_{0}=1$.

Theorem 4. Suppose $\operatorname{dim}\left(S_{0}\right)=\frac{1}{2} \operatorname{dim}(M)=n$ and that $\mathcal{N}$ is a $\operatorname{Ham}(M)$ orbit through a Lagrangian embedding, i.e. $\mathcal{N}$ is an isodrast in $\operatorname{Lag}\left(S_{0}, M\right)$. If $\mathbf{I}_{w}:=p\left(\mathcal{N} \times \operatorname{Vol}_{1}\left(S_{0}\right)\right)$, where $p: \operatorname{Lag}_{p w}\left(S_{0}, M\right) \rightarrow \operatorname{Lag}_{p w}(M)$, and $\mathcal{O}:=q(\mathcal{N})$, then the reduction $\left(\mathcal{O}, \Omega_{\text {red }}^{D}\right)$ of $\left(\operatorname{Emb}\left(S_{0}, M\right), \Omega^{D}\right)$ with respect to the $\operatorname{Diff}\left(S_{0}, \eta_{0}\right)$ action is symplectomorphic to the isodrast $\left(\mathbf{I}_{w}, \Omega\right)$ in $\operatorname{Lag}_{p w}(M)$.

Proof. By Proposition 11, we have a Ham $(M)$ equivariant diffeomorphism

$$
v: \operatorname{Lag}\left(S_{0}, M\right) / \operatorname{Diff}\left(S_{0}, \eta_{0}\right) \rightarrow \operatorname{Lag}_{p w}(M)
$$

which induces a diffeomorphism (also labeled by $v$ )

$$
v: \mathcal{O} \rightarrow \mathbf{I}_{w} .
$$


The pull back of $\Omega$ under this map is given by

$$
\begin{aligned}
\left(v^{*} \Omega\right)_{[i]}\left(\left[X_{1}\right],\left[X_{2}\right]\right) & =\Omega_{\left[\left(i, \eta_{0}\right)\right]}\left(\left[\left(X_{1}, 0\right)\right],\left[\left(X_{2}, 0\right)\right]\right) \\
& =\int_{S_{0}} \omega\left(X_{1}, X_{2}\right) \eta_{0}=\left(\Omega_{\mathrm{red}}^{D}\right)_{[i]}\left(\left[X_{1}\right],\left[X_{2}\right]\right) .
\end{aligned}
$$

So indeed, the symplectic quotient $\left(\mathcal{O}, \Omega_{\text {red }}^{D}\right)$ is symplectomorphic to the isodrast $\left(\mathbf{I}_{w}, \Omega\right)$.

Remark 12. This last theorem clarifies the relationship between Donaldson's symplectic structure $\Omega^{D}$ on $\operatorname{Emb}\left(S_{0}, M\right)$ and Weinstein's symplectic structure $\Omega^{W}$ on isodrasts in $\operatorname{Lag}_{p w}(M)$. Namely, the isodrasts $\left(\mathbf{I}_{w}, \Omega^{W}\right)$ can be viewed as symplectic quotients of $\left(\operatorname{Emb}\left(S_{0}, M\right), \Omega^{D}\right)$. And while Donaldson's and Weinstein's constructions were done heuristically, our constructions of the symplectic spaces $\left(\operatorname{Emb}\left(S_{0}, M\right), \Omega^{D}\right)$ and $\left(\mathbf{I}_{w}, \Omega\right)$ as well as the description of the relationship between them are rigourous.

Example 3. Let $(M, \omega)=\left(\mathbb{R}^{2}, d q \wedge d p\right)$ and $S_{0}=S^{1}$. Since $S^{1}$ is one dimensional Lag $\left(S^{1}, \mathbb{R}^{2}\right)$ $=\operatorname{Emb}\left(S^{1}, \mathbb{R}^{2}\right)$. However, since $H^{1}\left(S^{1}\right)$ is nontrivial, we have nontrivial isodrasts in $\operatorname{Emb}\left(S^{1}, \mathbb{R}^{2}\right)$ that can be described as follows.

Let $\beta=p d q$ denote the canonical 1-form on the plane. Given a map $i \in \operatorname{Emb}\left(S^{1}, \mathbb{R}^{2}\right)$, the action integral $A(i)$ of $i$ is defined as the integral of $\beta$ around $i\left(S^{1}\right)$ :

$$
A(i):=\int_{S^{1}} i^{*} \beta
$$

An isotopic deformation is Hamiltonian if and only if the action integrals are constant along the deformation (see Proposition 2.1 in [23]) ${ }^{1}$. The idea is as follows. Given two nearby loops in a symplectic manifold $(M, \omega)$, we can define the difference in their action integrals to be the integral of $-\omega$ over a cylindrical surface joining the two loops. This is well defined even when $\omega$ is not exact. Lagrangian submanifolds near a given $L \in \operatorname{Lag}(M)$ can be identified with graphs of 1 -forms in $T^{*} L$ by Theorem 1 . Two such graphs can be joined by a Hamiltonian deformation if and only if their corresponding 1-forms are cohomologous. If $\gamma^{\prime}$ is a small deformation of a loop $\gamma$ in the zero section corresponding to a deformation of the zero section to a Lagrangian submanifold $L^{\prime}=\operatorname{Graph}(\alpha), C$ denotes a cylinder joining $\gamma$ and $\gamma^{\prime}$, and if $\beta_{T^{*} L}$ denotes the canonical 1-form of the $T^{*} L$, then

$$
\int_{C}-\omega_{T^{*} L}=\int_{\gamma^{\prime}} \beta_{T^{*} L}=\int_{\gamma} \alpha
$$

So a small deformation of the zero section is the graph of an exact 1-form if and only if the difference in action integrals is 0 for all loops $\gamma$ and $\gamma^{\prime}$ in the zero section and the deformed image respectively. It follows that two graphs of 1 -forms can be joined by a Hamiltonian deformation if and only if the difference in action integrals remains constant for all loops in these Lagrangian submanifolds. Returning to our example, this means that the isodrasts in $\operatorname{Emb}\left(S^{1}, \mathbb{R}^{2}\right)$ consist of circle embeddings that can be joined by an isotopy that preserves action integrals, i.e. $\mathbf{H} \subset$ $\operatorname{Emb}\left(S^{1}, \mathbb{R}^{2}\right)$ is an isodrast if and only if it consists of isotopic circle embeddings and the map $A: \operatorname{Emb}\left(S^{1}, \mathbb{R}^{2}\right) \rightarrow \mathbb{R}$ sending $i$ to its action integral $A(i)$ is constant on $\mathbf{H}$.

On the circle we can take $\eta_{0}=\frac{d t}{2 \pi}$ so that $\operatorname{Diff}\left(S^{1}, \frac{d t}{2 \pi}\right)$ consists of rigid rotations of the circle. Let $\mathcal{O}:=q(\mathbf{H})$ be the image of an isodrast $\mathbf{H}$ in the orbit space. Each representative $X$ for a tangent vector $[X] \in T_{[i]} \mathcal{O}$ can be decomposed as $X=Z+Y$ where $Z$ is a normal to $i\left(S^{1}\right)$

\footnotetext{
${ }^{1}$ In fact, this was Weinstein's original motivation for the terminology "isodrast".
} 
and $Y$ is tangent to $i\left(S^{1}\right)$. It follows that if $\alpha_{X_{k}}=d h_{k}$ then the reduced symplectic structure is given by

$$
\begin{aligned}
\Omega_{\text {red }}^{D}\left(\left[X_{1}\right],\left[X_{2}\right]\right) & =\frac{1}{2 \pi}\left[\int_{0}^{2 \pi} \omega\left(Z_{1}, Y_{2}\right) d t+\omega\left(Y_{1}, Z_{2}\right)\right] d t=\frac{1}{2 \pi} \int_{0}^{2 \pi}\left[\mathcal{L}_{Y_{2}} h_{1} d t-\mathcal{L}_{Y_{1}} h_{2}\right] d t \\
& =\Omega\left(\left[\left(Z_{1},-\mathcal{L}_{Y_{1}} \frac{d t}{2 \pi}\right)\right],\left[\left(Z_{2},-\mathcal{L}_{Y_{2}} \frac{d t}{2 \pi}\right)\right]\right)=\Omega\left(\left[\left(X_{1}, 0\right)\right],\left[\left(X_{2}, 0\right)\right]\right) .
\end{aligned}
$$

\section{Weighted isotropic submanifolds}

The results of the last section suggest a way to generalize Weinstein's construction of a symplectic structure on spaces of weighted Lagrangian submanifolds to spaces of weighted isotropic submanifolds. In this section we will pursue this idea and thereby obtain a generalization of Theorem 4 suggested by Proposition 15.

Let $(M, \omega)$ be a symplectic manifold and $I_{0}$ a fixed compact oriented manifold with $\operatorname{dim} I_{0} \leq$ $\frac{1}{2} \operatorname{dim}(M)$.

\section{Notation}

Let Iso $\left(I_{0}, M\right)$ denote the set of isotropic embeddings of $I_{0}$ into $(M, \omega)$. That is,

$$
\operatorname{Iso}\left(I_{0}, M\right):=\left\{i \in \operatorname{Emb}\left(I_{0}, M\right) \mid i^{*} \omega=0\right\} .
$$

Similar to the discussion in Proposition 1, Theorem 3 can be used to equip Iso $\left(I_{0}, M\right)$ with a smooth manifold structure locally modeled on spaces $\Gamma_{\text {closed }}\left(i^{*} T M\right)$ for $i \in \operatorname{Iso}\left(I_{0}, M\right)$. For each $i \in \operatorname{Iso}\left(I_{0}, M\right)$, if $I:=i\left(I_{0}\right)$ then the sequence

$$
0 \longrightarrow \Gamma\left((T I)^{\omega}\right) \stackrel{f_{1}}{\longrightarrow} T_{i} \operatorname{Iso}\left(I_{0}, M\right) \stackrel{f_{2}}{\longrightarrow} Z^{1}\left(I_{0}\right) \longrightarrow 0
$$

where $f_{1}(Z)=Z \circ i$ and $f_{2}(X)=\alpha_{X}$, is an exact sequence.

Meanwhile, the atlas on Iso $\left(I_{0}, M\right)$ and the spaces $\Gamma_{\text {exact }}\left(i^{*} T M\right)$ for $i \in \operatorname{Iso}\left(I_{0}, M\right)$ define a foliation $\mathcal{E}$ of Iso $\left(I_{0}, M\right)$, whose leaves consist of $\operatorname{Ham}(M)$ orbits under the action

$$
\operatorname{Ham}(M) \circlearrowright \operatorname{Iso}\left(I_{0}, M\right): \quad \phi \cdot i:=\phi \circ i .
$$

Definition 11. The foliation $\mathcal{E}$ will be called the isodrastic foliation of Iso $\left(I_{0}, M\right)$, and each leaf of $\mathcal{E}$ will be called an isodrast in Iso $\left(I_{0}, M\right)$.

The group Diff $+\left(I_{0}\right)$ acts freely on Iso $\left(I_{0}, M\right)$ via

$$
a \cdot i:=i \circ a^{-1} \text {. }
$$

\section{Notation}

Set

$$
\operatorname{Iso}(M):=\operatorname{Iso}\left(I_{0}, M\right) / \operatorname{Diff}_{+}\left(I_{0}\right)
$$

If $p: \operatorname{Iso}\left(I_{0}, M\right) \rightarrow \operatorname{Iso}(M)$ denotes the projection to the quotient, then Iso $\left(I_{0}, M\right)$ is the total space of a principal $\operatorname{Diff}_{+}\left(I_{0}\right)$ bundle whose base Iso $(M)$ is identified with the set of oriented isotropic submanifolds in $M$ diffeomorphic to $I_{0}$. 


\section{Notation}

Let $\operatorname{Iso}_{w}\left(I_{0}, M\right)$ denote the product Iso $\left(I_{0}, M\right) \times \Omega_{1}^{k}\left(I_{0}\right)$ (where $\left.k=\operatorname{dim} I_{0}\right)$.

The group $\operatorname{Diff}_{+}\left(I_{0}\right)$ acts freely on $\operatorname{Iso}_{w}\left(I_{0}, M\right)$ by

$$
a \cdot(i, \eta):=\left(i \circ a^{-1},\left(a^{-1}\right)^{*} \eta\right) .
$$

\section{Notation}

Set

$$
\operatorname{Iso}_{w}(M):=\operatorname{Iso}_{w}\left(I_{0}, M\right) / \operatorname{Diff}_{+}\left(I_{0}\right) .
$$

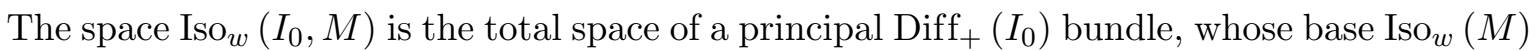
can be identified with the space of weighted isotropic submanifolds in $M$. The foliation $\mathcal{E}$ of Iso $\left(I_{0}, M\right)$ induces a foliation $\mathcal{E}_{w}$ of $\operatorname{Iso}_{w}\left(I_{0}, M\right)$ which descends to a foliation $\mathcal{F}_{w}$ of $\operatorname{Iso}_{w}(M)$.

Definition 12. We will call $\mathcal{E}_{w}$ the isodrastic foliation of $\operatorname{Iso}_{w}\left(I_{0}, M\right)$, and each leaf of $\mathcal{E}_{w}$ will be called an isodrast in $\operatorname{Iso}_{w}\left(I_{0}, M\right)$. Similarly, $\mathcal{F}_{w}$ will be called the isodrastic foliation of $\operatorname{Iso}_{w}(M)$, and each leaf of $\mathcal{F}_{w}$ will be called an isodrast in $\operatorname{Iso}_{w}(M)$.

The pointwise assignment

$$
\Omega_{(i, \eta)}\left(\left(X_{1}, \vartheta_{1}\right),\left(X_{2}, \vartheta_{2}\right)\right):=\int_{I_{0}}\left[\omega\left(X_{1}, X_{2}\right) \eta+h_{1} \vartheta_{2}-h_{2} \vartheta_{1}\right]
$$

where $\alpha_{X_{k}}=d h_{k}$ for $h_{k} \in C^{\infty}\left(I_{0}, \mathbb{R}\right)$ defines a basic 2-form on $\operatorname{Iso}_{w}\left(I_{0}, M\right)^{\mathcal{E}_{w}}$. It descends to a weak symplectic structure (also labeled $\Omega$ ) on $\operatorname{Iso}_{w}\left(I_{0}, M\right)^{\mathcal{F}_{w}}$. Here closedness and nondegeneracy of $\Omega$ on $\mathbf{I}_{w}$ follow from using the local model of isotropic submanifolds afforded by Theorem 3, and the fact that in such a model the symplectic form along the zero section in $T^{*} I \oplus \Gamma\left(T I^{\omega} / T I\right)$ is given by $\omega_{T^{*} I}+\omega_{\mathbb{R}^{2 n}}$ where $\omega_{\mathbb{R}^{2 n}}$ is the standard symplectic vector space structure on the fibers of $\Gamma\left(T I^{\omega} / T I\right)$.

\section{Notation}

Let $\operatorname{Iso}_{p w}\left(I_{0}, M\right)$ denote the product Iso $\left(I_{0}, M\right) \times \operatorname{Vol}_{1}\left(I_{0}\right)$ and set

$$
\operatorname{Iso}_{p w}(M):=\operatorname{Iso}_{p w}\left(I_{0}, M\right) / \operatorname{Diff}_{+}\left(I_{0}\right) \text {. }
$$

$\operatorname{Iso}_{p w}(M)$ is an open subset of $\operatorname{Iso}_{w}(M)$, and so is a smooth manifold locally modeled on spaces $\Gamma_{\text {closed }}\left(i^{*} T M\right) \oplus \Omega_{0}^{k}\left(I_{0}\right) /\left\{\left(Y, \mathcal{L}_{Y} \eta\right) \mid Y \in \mathfrak{X}\left(I_{0}\right)\right\}$ for $(i, \eta) \in \operatorname{Isopw}_{p w}\left(I_{0}, M\right)$. If $I_{0}$ is equipped with a fixed volume form $\eta_{0}$ satisfying $\int_{I_{0}} \eta_{0}=1$, then as in Proposition 11 there is a natural $\operatorname{Ham}(M)$ equivariant diffeomorphism $v: \operatorname{Iso}\left(I_{0}, M\right) / \operatorname{Diff}\left(I_{0}, \eta_{0}\right) \rightarrow \operatorname{Iso}_{p w}(M)$. This leads to the following generalization of Theorem 4 :

Theorem 5. Suppose that $\mathcal{N}$ is a Ham $(M)$ orbit through an isotropic embedding, i.e. $\mathcal{N}$ is an isodrast in Iso $\left(S_{0}, M\right)$. If $\mathbf{I}_{w}:=p\left(\mathcal{N} \times \operatorname{Vol}_{1}\left(S_{0}\right)\right)$ and $\mathcal{O}:=q(\mathcal{N})$, where $p: \operatorname{Iso}_{p w}\left(L_{0}, M\right) \rightarrow$ $\operatorname{Iso}_{p w}(M)$ and $q: \operatorname{Emb}\left(S_{0}, M\right) \rightarrow \operatorname{Emb}\left(S_{0}, M\right) / \operatorname{Diff}\left(S_{0}, \eta_{0}\right)$ are the projections to the quotients, then the reduction $\left(\mathcal{O}, \Omega_{\text {red }}^{D}\right)$ of $\left(\operatorname{Emb}\left(S_{0}, M\right), \Omega^{D}\right)$ with respect to the $\operatorname{Diff}\left(S_{0}, \eta_{0}\right)$ action is symplectomorphic to the isodrast $\left(\mathbf{I}_{w}, \Omega\right)$ in $\operatorname{Iso}_{p w}(M)$.

The proof is completely analogous to that of Theorem 4. 


\section{Coadjoint orbits of Ham $(M)$}

In this section we will extend a $\operatorname{Ham}(M)$ moment map written down by Weinstein (see Theorem 5.1 in [23]), which maps each symplectic leaf $\left(\mathbf{I}_{w}, \Omega\right) \subset \operatorname{Lag}_{p w}(M)$ onto a coadjoint orbit of a central extension of $\operatorname{Ham}(M)$. The result will be an identification of isodrasts consisting of positive weighted isotropic submanifolds with coadjoint orbits of $\operatorname{Ham}(M)$ (when $M$ is compact).

Suppose that $(M, \omega)$ is compact. The Lie algebra of $G=\operatorname{Ham}(M)$ can be identified with the space

$$
\mathfrak{g}=\left\{f \in C^{\infty}(M, \mathbb{R}) \mid \int_{M} f \frac{\omega^{n}}{n !}=0\right\} \simeq C^{\infty}(M, \mathbb{R}) / \mathbb{R}
$$

Each positive weighted isotropic submanifold $(I, \rho) \in \operatorname{Iso}_{p w}(M)$ defines a element of the dual $\mathfrak{g}^{*}$ by integration:

$$
f \mapsto \int_{I} f \rho .
$$

Theorem 6. Let $\mathbf{I}_{w}$ be an isodrast in $\operatorname{Iso}_{p w}(M)$. Then the Ham $(M)$ action on $\left(\mathbf{I}_{w}, \Omega\right)$ defined by $\phi \cdot(I, \rho):=\left(\phi(I),\left(\phi^{-1}\right)^{*} \rho\right)$ is Hamiltonian with moment map $\Phi: \mathbf{I}_{w} \rightarrow \mathfrak{g}^{*}$ given by

$$
(I, \rho) \mapsto\left(f \mapsto \int_{I} f \rho\right)
$$

Moreover, the map $\Phi$ is a symplectomorphism onto a coadjoint orbit in $\mathfrak{g}^{*}$ equipped with the usual Kirillov-Kostant-Souriau form.

Proof. We will first show that the $\operatorname{Ham}(M)$ action is symplectic. If $(I, \rho)=[(i, \eta)]$, then the $\operatorname{Ham}(M)$ action on $\mathbf{I}_{w}$ can be written as

$$
\phi \cdot[(i, \eta)]:=[(\phi \circ i, \eta)] .
$$

It follows that

$$
\begin{aligned}
\left(l_{\phi}^{*} \Omega\right)_{[(\phi \cdot i, \eta)]}\left(\left[\left(X_{1}, \vartheta_{1}\right)\right],\left[\left(X_{2}, \vartheta_{2}\right)\right]\right) & =\int_{I_{0}}\left[\omega\left(\mathrm{d} \phi \cdot X_{1}, \mathrm{~d} \phi \cdot X_{2}\right) \eta+h_{1} \vartheta_{2}-h_{2} \vartheta_{1}\right] \\
& =\int_{I_{0}}\left[\omega\left(X_{1}, X_{2}\right) \eta+h_{1} \vartheta_{2}-h_{2} \vartheta_{1}\right] \\
& =\Omega_{[(i, \eta)]}\left(\left[\left(X_{1}, \vartheta_{1}\right)\right],\left[\left(X_{2}, \vartheta_{2}\right)\right]\right) .
\end{aligned}
$$

To check the moment map condition, we first note that each tangent vector $\xi \in T_{[(i, \eta)]} \mathbf{I}_{w}$ has a unique representative of the form $(X, 0)$. If $f \in \mathfrak{g}$ then the generating vector field $f_{\mathbf{I}_{w}}$ assigns to each point $[(i, \eta)] \in \mathbf{I}_{w}$ the tangent vector $\left[\left(X_{f} \circ i, 0\right)\right]$ where $X_{f}$ is the Hamiltonian vector field on $M$ corresponding to the Hamiltonian $f$. If $[(X, 0)] \in T_{[(i, \eta)]} \mathbf{I}_{w}$ is a tangent vector to the isodrast, then choose an extension of $X$ to a Hamiltonian vector field $Z$ defined on a neighbourhood of $I=i\left(I_{0}\right)$. It follows that

$$
\begin{aligned}
\mathrm{d}\langle\Phi, f\rangle_{[(i, \eta)]}([(X, 0)]) & =\int_{I_{0}} i^{*} \mathcal{L}_{Z} f \eta=\int_{I_{0}} i^{*} \omega\left(X_{f}, Z\right) \eta \\
& =\int_{I_{0}} \omega\left(X_{f} \circ i, X\right) \eta=\Omega_{[(i, \eta)]}\left(f_{\mathbf{I}_{w}},[(X, 0)]\right) .
\end{aligned}
$$


To check that the map $\Phi: \mathbf{I}_{w} \rightarrow \mathfrak{g}^{*}$ is $\operatorname{Ham}(M)$ equivariant we observe that

$$
\begin{aligned}
\langle\Phi(\phi \cdot[(i, \eta)]), f\rangle & =\int_{I_{0}} i^{*} \phi^{*} f \eta=\int_{I_{0}} i^{*}(f \circ \phi) \eta \\
& =\left\langle\Phi([(i, \eta)]), \operatorname{Ad}_{\phi^{-1}} f\right\rangle=\left\langle\operatorname{Ad}_{\phi}^{*} \Phi([(i, \eta)]), f\right\rangle .
\end{aligned}
$$

As for the image of $\Phi$, since the stabilizers at points $(I, \rho) \in \mathbf{I}_{w}$ and $\Phi(I, \rho) \in \mathfrak{g}^{*}$ are given by

$$
\operatorname{Stab}_{(I, \rho)} \operatorname{Ham}(M)=\left\{\phi \in \operatorname{Ham}(M) \mid \phi(I)=I,\left(\phi^{-1}\right)^{*} \rho=\rho\right\}=\operatorname{Stab}_{\Phi(I, \rho)} \operatorname{Ham}(M),
$$

the map $\Phi$ maps the isodrast $\mathbf{I}_{w}$ diffeomorphically onto a coadjoint orbit. Meanwhile, if $\left[\left(X_{1}, 0\right)\right]$ and $\left[\left(X_{2}, 0\right)\right]$ are tangent vectors in $T_{[(i, \eta)]} \mathbf{I}_{w}$, and $Z_{f_{1}}$ and $Z_{f_{2}}$ are extensions of $X_{1}$ and $X_{2}$ respectively to Hamiltonian vector fields defined on a neighbourhood of $I=i\left(I_{0}\right)$, then the pullback under $\Phi$ of the usual Kirillov-Kostant-Souriau form $\Omega_{\mathrm{KKS}}$ is given by

$$
\begin{aligned}
\left(\Phi^{*} \Omega_{\mathrm{KKS}}\right)_{[(i, \eta)]}\left(\left[\left(X_{1}, 0\right)\right],\left[\left(X_{2}, 0\right)\right]\right) & =-\int_{I_{0}} i^{*}\left\{f_{1}, f_{2}\right\} \eta \\
& =\int_{I_{0}} i^{*} \omega\left(Z_{f_{1}}, Z_{f_{2}}\right) \eta=\Omega_{[(i, \eta)]}\left(\left[\left(X_{1}, 0\right)\right],\left[\left(X_{2}, 0\right)\right]\right) .
\end{aligned}
$$

Remark 13. This last theorem makes rigourous the heuristic moment map written down by Weinstein. Also, it extends it to a map identifying isodrasts in the space of positive weighted isotropic submanifolds with coadjoint orbits in the dual of the Lie algebra of the group of Hamiltonian symplectomorphisms.

\section{Poisson structures}

In this section we will define a Poisson algebra $(\mathcal{A},\{\cdot, \cdot\})$ for a subalgebra $\mathcal{A} \subset C^{\infty}(M)$ of smooth functions on a smooth manifold $M$. Such a manifold will be called an $\mathcal{A}$-Poisson manifold if there are enough Hamiltonian vector fields in a sense we will specify. We define what a leaf of such an $\mathcal{A}$-Poisson manifold is. We then show that reductions of $\operatorname{Emb}\left(S_{0}, M\right)$ consisting of positive weighted isotropic submanifolds are symplectic leaves of a Poisson structure.

\section{Notation}

Let $C_{\int}^{\infty}\left(\operatorname{Emb}\left(S_{0}, M\right)\right)$ denote the set of maps $F: \operatorname{Emb}\left(S_{0}, M\right) \rightarrow \mathbb{R}$ such that for all $i_{0} \in$ $\operatorname{Emb}\left(S_{0}, M\right)$ there exists a $c^{\infty}$-open neighbourhood $U$ of $i_{0}$, a map $A \in C^{\infty}\left(\mathbb{R}^{n}, \mathbb{R}\right)$, and smooth functions $h_{1}, \ldots, h_{n} \in C^{\infty}(W, \mathbb{R})$ defined on a neighbourhood $W$ of $i_{0}\left(S_{0}\right)$ so that

$$
F(i)=A\left(\int_{S_{0}}\left(i^{*} h_{1}\right) \eta_{0}, \ldots, \int_{S_{0}}\left(i^{*} h_{n}\right) \eta_{0}\right)
$$

for all $i \in U$.

Since functions in $C_{\int}^{\infty}\left(\operatorname{Emb}\left(S_{0}, M\right)\right)$ locally amount to integrating functions against $\eta_{0}$, the set $C_{\int}^{\infty}\left(\operatorname{Emb}\left(S_{0}, M\right)\right)$ is a subalgebra of $C^{\infty}\left(\operatorname{Emb}\left(S_{0}, M\right), \mathbb{R}\right)$.

Remark 14. The algebra of functions $C_{\int}^{\infty}\left(\operatorname{Emb}\left(S_{0}, M\right)\right)$ was chosen with the axioms of differential structures in mind (see [13]; also cf. Section 3 in [2]). A differential structure on a topological space $Q$ is a set $C^{\infty}(Q)$ of continuous functions on $Q$ with the following properties:

1. The topology of $Q$ is generated by sets of the form $F^{-1}(V)$ where $F \in C^{\infty}(Q)$ and $V$ is an open subset of $\mathbb{R}$. 
2. If $B \in C^{\infty}\left(\mathbb{R}^{m}, \mathbb{R}\right)$ and $F_{1}, \ldots, F_{m} \in C^{\infty}(Q)$ then $B\left(F_{1}, \ldots, F_{m}\right) \in C^{\infty}(Q)$.

3. If $F: Q \rightarrow \mathbb{R}$ is a function such that, for every $x \in Q$ there is an open neighbourhood $U$ of $x$ in $Q$ and a function $F_{U} \in C^{\infty}(Q)$ such that $\left.F\right|_{U}=F_{U}$, then $F \in C^{\infty}(Q)$.

A topological space $Q$ together with a differential structure $C^{\infty}(Q)$ is called a differential space. When $\operatorname{Emb}\left(S_{0}, M\right)$ is equipped with the topology $\mathcal{T}$ generated by sets of the form $F^{-1}(V)$ where $F \in C_{\int}^{\infty}\left(\operatorname{Emb}\left(S_{0}, M\right)\right)$ and $V$ is open in $\mathbb{R}$, then the topological space $\left(\operatorname{Emb}\left(S_{0}, M\right), \mathcal{T}\right)$ together with $C_{\int}^{\infty}\left(\operatorname{Emb}\left(S_{0}, M\right)\right)$ defines a differential space.

Proposition 16. For every $F \in C_{\int}^{\infty}\left(\operatorname{Emb}\left(S_{0}, M\right)\right)$, the local assignments given by $v_{F}(i):=$ $\left.\sum_{j=1}^{n} \frac{\partial A}{\partial y_{j}} X_{h_{j}}\right|_{i\left(S_{0}\right)} \circ i$ on each neighbourhood $U$ define a unique vector field $v_{F}$ on $\operatorname{Emb}\left(S_{0}, M\right)$ satisfying $d F=\imath\left(v_{F}\right) \Omega^{D}$.

Proof. We will first compute the exterior derivative of a map in $C_{\int}^{\infty}\left(\operatorname{Emb}\left(S_{0}, M\right)\right)$ locally. Suppose that $F \in C_{\int}^{\infty}\left(\operatorname{Emb}\left(S_{0}, M\right)\right)$ can be written as in $(9)$ on a $c^{\infty}$-open neighbourhood $U$ of $i_{0} \in \operatorname{Emb}\left(S_{0}, M\right)$. Let $X \in T_{i_{0}} \operatorname{Emb}\left(S_{0}, M\right)$ be a tangent vector at $i_{0}$. Choose a vector field $Z$ defined on a neighbourhood of $i\left(S_{0}\right)$ such that $Z \circ i=X$. Such a $Z$ can be viewed as a vector field $v$ on $U$ (or perhaps on a $c^{\infty}$-open subset $V \subset U$ ), assigning $Z \circ i$ to each $i \in U$. If

$$
F(i)=A\left(\int_{S_{0}}\left(i^{*} h_{1}\right) \eta_{0}, \ldots, \int_{S_{0}}\left(i^{*} h_{n}\right) \eta_{0}\right)
$$

on $U$ for some $A \in C^{\infty}\left(\mathbb{R}^{n}, \mathbb{R}\right)$ and smooth functions $h_{1}, \ldots, h_{n} \in C^{\infty}(W, \mathbb{R})$ defined on a neighbourhood $W$ of $i_{0}\left(S_{0}\right)$, then the pointwise exterior derivative of $F$ in the direction of $v$ is given by

$$
d F_{i}\left(v_{i}\right)=\sum_{j=1}^{n} \frac{\partial A}{\partial y_{j}} \int_{S_{0}}\left(i^{*} \mathcal{L}_{Z} h_{j}\right) \eta_{0}
$$

It follows that the vector field $v_{F} \in \mathfrak{X}(U)$, defined by $v_{F}(i):=\sum_{j=1}^{n} \frac{\partial A}{\partial y_{j}} X_{h_{j}} \circ i$ (where $X_{h_{j}}$ is the Hamiltonian vector field with Hamiltonian $\left.h_{j}\right)$, satisfies $d F=\imath\left(v_{F}\right) \Omega^{D}$ on $U$. In fact, if $U_{1}$ and $U_{2}$ are $c^{\infty}$-open neighbourhoods in $\operatorname{Emb}\left(S_{0}, M\right)$ with nonempty intersection, and

$$
\begin{aligned}
& F(i)=A\left(\int_{S_{0}}\left(i^{*} h_{1}\right) \eta_{0}, \ldots, \int_{S_{0}}\left(i^{*} h_{n}\right) \eta_{0}\right) \text { on } U_{1}, \\
& F(i)=B\left(\int_{S_{0}}\left(i^{*} g_{1}\right) \eta_{0}, \ldots, \int_{S_{0}}\left(i^{*} g_{m}\right) \eta_{0}\right) \text { on } U_{2},
\end{aligned}
$$

then on $U_{1} \cap U_{2}$

$$
\begin{aligned}
0 & =d\left(A\left(\int_{S_{0}}\left(i^{*} h_{1}\right) \eta_{0}, \ldots, \int_{S_{0}}\left(i^{*} h_{n}\right) \eta_{0}\right)-B\left(\int_{S_{0}}\left(i^{*} g_{1}\right) \eta_{0}, \ldots, \int_{S_{0}}\left(i^{*} g_{m}\right) \eta_{0}\right)\right)(v) \\
& =\Omega^{D}\left(\sum_{j=1}^{n} \frac{\partial A}{\partial y_{j}} X_{h_{j}} \circ i-\sum_{l=1}^{m} \frac{\partial B}{\partial z_{l}} X_{g_{l}} \circ i, v\right) .
\end{aligned}
$$

So by the nondegeneracy of $\Omega^{D}$, the local assignments $v_{F}(i):=\sum_{j=1}^{n} \frac{\partial A}{\partial y_{j}} X_{h_{j}} \circ i$ coincide on overlapping regions and so define a vector field $v_{F}$ on $\operatorname{Emb}\left(S_{0}, M\right)$ satisfying $d F=\imath\left(v_{F}\right) \Omega^{D}$. Uniqueness of the Hamiltonian vector field $v_{F}$ also follows from nondegeneracy of $\Omega^{D}$. 
Definition 13. Let $M$ be a smooth manifold. A subalgebra $\mathcal{A} \subset C^{\infty}(M, \mathbb{R})$ of smooth functions together with a Lie structure $[\cdot, \cdot]$ will be called a Poisson algebra if

$$
[F G, H]=F[G, H]+[F, H] G .
$$

In this case the bracket $[\cdot, \cdot]$ will be called a Poisson bracket. If $(\mathcal{A},[\cdot, \cdot])$ is a Poisson algebra, we will say that $M$ is an $\mathcal{A}$-Poisson manifold.

Define a skew-symmetric bilinear structure $\{\cdot, \cdot\}_{\operatorname{Emb}\left(S_{0}, M\right)}$ on $C_{\int}^{\infty}\left(\operatorname{Emb}\left(S_{0}, M\right)\right)$ via

$$
\{F, G\}_{\operatorname{Emb}\left(S_{0}, M\right)}:=-\Omega^{D}\left(v_{F}, v_{G}\right) .
$$

Proposition 17. The subalgebra $C_{\int}^{\infty}\left(\operatorname{Emb}\left(S_{0}, M\right)\right)$ together with $\{\cdot, \cdot\}_{\operatorname{Emb}\left(S_{0}, M\right)}$ is a Poisson algebra.

Proof. $\{\cdot, \cdot\}_{\operatorname{Emb}\left(S_{0}, M\right)}$ satisfies Jacobi's identity pointwise since the Poisson bracket $\{\cdot, \cdot\}_{M}$ on $M$ does, and is a derivation in the first argument for the same reason. (Here $\{\cdot, \cdot\}_{M}$ is defined by $\{f, g\}_{M}:=-\omega\left(X_{f}, X_{g}\right)$ for $f, g \in C^{\infty}(M, \mathbb{R})$.)

By taking restrictions of functions in $C_{\int}^{\infty}\left(\operatorname{Emb}\left(S_{0}, M\right)\right)$ to Iso $\left(S_{0}, M\right)$ we obtain an algebra $C_{\int}^{\infty}$ (Iso $\left.\left(S_{0}, M\right)\right)$ of smooth functions on Iso $\left(S_{0}, M\right)$. For each $F \in C_{\int}^{\infty}\left(\operatorname{Iso}\left(S_{0}, M\right)\right)$ and $i \in$ Iso $\left(S_{0}, M\right)$ the vector $v_{F}(i)$ is tangent to Iso $\left(S_{0}, M\right)$ at $i$. Since the functions in $C_{\int}^{\infty}\left(\right.$ Iso $\left.\left(S_{0}, M\right)\right)$ are $\operatorname{Diff}\left(S_{0}, \eta_{0}\right)$ invariant, the algebra $C_{\int}^{\infty}\left(\operatorname{Iso}\left(S_{0}, M\right)\right)$ descends to an algebra of functions $C_{\int}^{\infty}\left(\operatorname{Iso}_{p w}(M)\right)$ on $\operatorname{Iso}_{p w}(M)$.

\section{Convention}

In what follows, we will use the same letter $F$ to denote a function in $C_{\int}^{\infty}\left(\operatorname{Emb}\left(S_{0}, M\right)\right)$, its restriction to Iso $\left(S_{0}, M\right)$, as well as the corresponding map on the quotient $\operatorname{Iso}_{p w}(M)$ to avoid introducing more notation.

For $F, G \in C_{\int}^{\infty}\left(\operatorname{Iso}_{p w}(M)\right)$ and $[i] \in \operatorname{Iso}_{p w}(M)$ define

$$
\{F, G\}_{\operatorname{Iso} p w_{p(M)}}([i]):=-\Omega_{i}^{D}\left(v_{F}(i), v_{G}(i)\right)=-\left(\Omega_{\mathrm{red}}^{D}\right)_{[i]}\left(\mathrm{d} r\left(v_{F}\right), \mathrm{d} r\left(v_{G}\right)\right),
$$

where $r:$ Iso $\left(S_{0}, M\right) \rightarrow \operatorname{Iso}_{p w}(M)$ is the projection to the quotient. Then the subalgebra $C_{\int}^{\infty}\left(\operatorname{Iso}_{p w}(M)\right)$ together with $\{\cdot, \cdot\}_{\operatorname{Iso}_{p w}(M)}$ is a Poisson algebra, which means $\operatorname{Iso}_{p w}(M)$ is a $C_{\int}^{\infty}\left(\operatorname{Iso}_{p w}(M)\right)$-Poisson manifold.

Definition 14. Let $(M, \mathcal{A},\{\cdot, \cdot\})$ be an $\mathcal{A}$-Poisson manifold. Suppose that for every $F \in \mathcal{A}$ there exists a unique vector field $X_{F}$ on $M$ such that $\mathrm{d} G\left(X_{F}\right)=\{F, G\}$ for every $G \in \mathcal{A}$. The collection of vectors

$$
\mathcal{C}_{x}:=\left\{X_{F}(x) \in T_{x} M \mid F \in \mathcal{A}\right\}
$$

at each $x \in M$ defines a distribution $\mathcal{C}$ on $M$. A maximal integral manifold $\mathcal{M}$ of $\mathcal{C}$ will be called a leaf of the $\mathcal{A}$-Poisson manifold $M$.

Remark 15. In finite dimensions the distribution $\mathcal{C}$ is integrable. In infinite dimensions it might be possible to prove an analogue of Frobenius' theorem for some spaces of mappings and apply it to our spaces.

Remark 16. The first condition in the previous definition is satisfied within the subalgebra $C_{\int}^{\infty}\left(\operatorname{Iso}_{p w}(M)\right)$ but not in $C_{\int}^{\infty}\left(\operatorname{Emb}\left(S_{0}, M\right)\right)$ because of the directions generated by the $\operatorname{Diff}\left(S_{0}, \eta_{0}\right)$ action. 
For each $F \in C_{\int}^{\infty}\left(\operatorname{Iso}_{p w}(M)\right)$ we have that $\mathrm{d} r\left(v_{F}\right)$ is the unique vector field on $\operatorname{Iso}_{p w}(M)$ satisfying $d F=\imath\left(\mathrm{d} r\left(v_{F}\right)\right) \Omega_{\text {red }}^{D}$ (uniqueness follows from the nondegeneracy of $\Omega_{\text {red }}^{D}$ on $\left.\operatorname{Iso}_{p w}(M)^{\mathcal{F}_{p w}}\right)$. It follows that if $F \in C_{\int}^{\infty}\left(\operatorname{Iso}_{p w}(M)\right)$ then $\mathrm{d} G\left(X_{F}\right)=\{F, G\}_{\operatorname{Iso}_{p w}(M)}$ for all $G \in C_{\int}^{\infty}\left(\operatorname{Iso}_{p w}(M)\right)$. On the $C_{\int}^{\infty}\left(\operatorname{Iso}_{p w}(M)\right)$-Poisson manifold $\operatorname{Iso}_{p w}(M)$, the distribution $\mathcal{C}$ is given by the collection of vectors

$$
\mathcal{C}_{[i]}:=\left\{\mathrm{d} r\left(v_{F}\right)([i]) \in T_{[i]} \operatorname{Iso}_{p w}(M) \mid F \in C_{\int}^{\infty}\left(\operatorname{Iso}_{p w}\left(S_{0}, M\right)\right)\right\}
$$

at each $[i] \in \operatorname{Iso}_{p w}(M)$.

Theorem 7. Suppose that $\mathcal{N}$ is a $\operatorname{Ham}(M)$ orbit through an isotropic embedding in $\operatorname{Emb}\left(S_{0}, \eta_{0}\right)$, i.e., $\mathcal{N}$ is an isodrast in Iso $\left(S_{0}, M\right)$, with $\mathcal{O}:=q(\mathcal{N})$ where the mapping $q: \operatorname{Emb}\left(S_{0}, M\right) \rightarrow$ $\operatorname{Emb}\left(S_{0}, M\right) / \operatorname{Diff}\left(S_{0}, \eta_{0}\right)$ is the projection to the quotient. Then the reduction $\left(\mathcal{O}, \Omega_{\mathrm{red}}^{D}\right)$ is a symplectic leaf of the $C_{\int}^{\infty}\left(\operatorname{Iso}_{p w}(M)\right)$-Poisson manifold $\operatorname{Iso}_{p w}(M)$.

Proof. We will first show that any maximal integral manifold of $\mathcal{C}$ is a $\operatorname{Ham}(M)$ orbit in $\operatorname{Iso}_{p w}(M)$ under the action $\phi \cdot[i]:=[\phi \circ i]$. The manifold Iso $\left(S_{0}, M\right)$ is the total space of a principal Diff $\left(S_{0}, M\right)$ bundle over Iso $p w(M)$. Each vector field $v_{F}$ on Iso $\left(S_{0}, M\right)$ with Hamiltonian $F \in C_{\int}^{\infty}$ (Iso $\left.\left(S_{0}, M\right)\right)$ is locally of the form $v_{F}(i)=\sum_{j=1}^{n} \frac{\partial A}{\partial y_{j}} X_{h_{j}} \circ i$. It follows that curves in $\operatorname{Iso}_{p w}(M)$ are everywhere tangent to the distribution $\mathcal{C}$ if and only if they belong to a $\operatorname{Ham}(M)$ orbit. So the symplectic quotient $\left(\mathcal{O}:=q(\mathcal{N}), \Omega_{\text {red }}^{D}\right)$ is a leaf of the $C_{\int}^{\infty}\left(\operatorname{Iso}_{p w}(M)\right)$-Poisson manifold $\operatorname{Iso}_{p w}(M)$.

Remark 17. By now we have revealed three different faces of isodrasts in $\operatorname{Iso}_{p w}(M)$. Namely, isodrasts consisting of positively weighted isotropic submanifolds can be identified with symplectic reductions of $\left(\operatorname{Emb}\left(S_{0}, M\right), \Omega^{D}\right)$, with coadjoint orbits of $\operatorname{Ham}(M)$, and with symplectic leaves of the $C_{\int}^{\infty}\left(\operatorname{Iso}_{p w}(M)\right)$-Poisson manifold $\operatorname{Iso}_{p w}(M)$.

\section{Momentum weighted Lagrangian submanifolds}

In this section we will discuss a kinematic interpretation of isodrasts in Lag $(M)$ to motivate a different choice of "weightings" to obtain a symplectic structure.

Let us view points in an isodrast $\mathbf{I} \subset \operatorname{Lag}(M)$ as configurations of a submanifold constrained to move in I. What are the possible velocities? By Proposition 5 and the description of the isodrastic foliation $\mathcal{F}$, we know that the tangent bundle of $\mathbf{I}$ can be described by

$$
T \mathbf{I}=\left\{(L, d h) \in \mathbf{I} \times B^{1}(L) \mid L \in \mathbf{I}\right\} .
$$

Thus the velocities at a configuration $L \in \mathbf{I}$ correspond to functions on $L$ modulo constants, i.e. to elements of $C^{\infty}(L, \mathbb{R}) / \mathbb{R}$. The conjugate momenta to configurations in $\mathbf{I}$ (i.e. cotangent vectors) should be linear functionals from $C^{\infty}(L, \mathbb{R}) / \mathbb{R}$ to $\mathbb{R}$, that are in 1-1 correspondence with $C^{\infty}(L, \mathbb{R}) / \mathbb{R}$. This expectation that the cotangent fibers should be the "same size" as $C^{\infty}(L, \mathbb{R}) / \mathbb{R}$ reflects a physical expectation that all momenta should be accessible by motions of particles in the configuration space, and that all such motions can be assigned momenta. In any case, integration against $n$-forms in $\Omega_{0}^{n}(L)$ certainly fits the above description. This motivates the following definition:

Definition 15. Let $\mathbf{I}$ be an isodrast in $\operatorname{Lag}(M)$. A momentum weighting of a Lagrangian submanifold $L \in \mathbf{I}$ is a top degree form $\chi$ on $L$ satisfying $\int_{L} \chi=0$. Pairs $(L, \chi)$ will be called momentum weighted Lagrangian submanifolds. 


\section{Notation}

Let $\operatorname{Lag}_{m w}\left(L_{0}, M\right)$ denote the product $\operatorname{Lag}\left(L_{0}, M\right) \times \Omega_{0}^{n}\left(L_{0}\right)$ and $\operatorname{Lag}_{m w}(M)$ the quotient of $\operatorname{Lag}_{m w}\left(L_{0}, M\right)$ by $\operatorname{Diff}_{+}\left(L_{0}\right)$ under the action $a \cdot(i, \chi):=\left(i \circ a^{-1},\left(a^{-1}\right)^{*} \chi\right)$.

As in the case of weighted Lagrangian submanifolds, the canonical projection from $\operatorname{Lag}_{m w}(M)$ to $\operatorname{Lag}(M)$ pulls back the foliation $\mathcal{F}$ to a foliation $\mathcal{F}_{m w}$ on $\operatorname{Lag}_{m w}(M)$, whose leaves $\mathbf{I}_{m w}$ are $\operatorname{Ham}(M)$ orbits under the action

$$
\operatorname{Ham}(M) \circlearrowright \operatorname{Lag}_{m w}(M): \phi \cdot[(i, \chi)]:=[(\phi \circ i, \chi)] .
$$

Definition 16. We will call $\mathcal{F}_{m w}$ the isodrastic foliation of $\operatorname{Lag}_{m w}(M)$ and each leaf of $\mathcal{F}_{m w}$ will be called an isodrast in $\operatorname{Lag}_{m w}(M)$.

The tangent space to an isodrast $\mathbf{I}_{m w}$ at a point $[(i, \chi)]$ is given by

$$
T_{[(i, \nu)]} \mathbf{I}_{m w}=\left\{(X, \vartheta) \in \Gamma\left(i^{*} T M\right) \times \Omega_{0}^{n}\left(L_{0}\right) \mid \alpha_{X} \in B^{1}\left(L_{0}\right)\right\} /\left\{\left(Y, \mathcal{L}_{Y} \eta\right) \mid Y \in \mathfrak{X}\left(L_{0}\right)\right\} .
$$

Theorem 8. The bilinear map

$$
\Omega_{[(i, \chi)]}\left(\left[\left(X_{1}, \vartheta_{1}\right)\right],\left[\left(X_{2}, \vartheta_{2}\right)\right]\right):=\int_{L_{0}}\left[\omega\left(X_{1}, X_{2}\right) \chi+h_{1} \vartheta_{2}-h_{2} \vartheta_{1}\right]
$$

where $\alpha_{X_{k}}=d h_{k}$, defines an exact weak symplectic structure on $\mathbf{I}_{m w}$ satisfying $\Omega=-d \Theta$ where

$$
\Theta_{[(i, \chi)]}([(X, \vartheta)]):=\int_{L_{0}} h \chi
$$

and $\alpha_{X}=d h$.

Proof. We will compute the exterior derivative of $\Theta$ locally in charts. That is, be means of a symplectic embedding $\lambda_{(i, \chi)}: T^{*} L_{0} \supset W_{(i, \chi)} \rightarrow M$ chosen in defining a chart $\left(U_{(i, \chi)}, \varphi_{(i, \chi)}\right)$ on $\operatorname{Lag}_{m w}(M)$, we can assume that $M=T^{*} L_{0}$ and that each tangent vector in $T_{[(i, \chi)]} \mathbf{I}_{m w}$ is represented by a pair $(X, \vartheta)$ where $\alpha_{X} \in B^{1}\left(L_{0}\right)$ and $X$ is tangent to the cotangent fibers. Given a tangent vector $\xi=(X, \vartheta) \in T_{[(i, \chi)]} \mathbf{I}_{m w}$, we can extend it to a vector field on $\mathbf{I}_{m w}$ (also labeled $\xi$ ) in the following way. Let $Z_{f}$ denote the Hamiltonian vector field defined on $T^{*} L_{0}$ satisfying $\imath\left(Z_{f}\right) \omega=\pi^{*} \alpha_{X}$ where $\alpha_{X}=d h$ and $f=\pi^{*} h$. Define $\xi$ to be the vector field on $\mathbf{I}_{m w}$ that assigns $\xi\left(\left[i^{\prime}, \nu^{\prime}\right]\right)=\left(Z_{f} \circ i^{\prime}, \vartheta\right)$ to nearby points $\left[\left(i^{\prime}, \chi^{\prime}\right)\right]$. So given tangent vectors $\xi_{1}$ and $\xi_{2}$ in $T_{[(i, \chi)]} \mathbf{I}_{m w}$, extend them to vector fields (also labeled $\xi_{1}$ and $\xi_{2}$ ) as just described. Then

$$
\begin{aligned}
d \Theta_{[(i, \nu)]}\left(\xi_{1}, \xi_{2}\right)= & {\left[\xi_{1} \Theta\left(\xi_{2}\right)-\xi_{2} \Theta\left(\xi_{1}\right)-\Theta\left(\left[\xi_{1}, \xi_{2}\right]\right)\right]_{[(i, \nu)]} } \\
= & \int_{L_{0}}\left[\left(i^{*} L_{X_{f_{1}}} f_{2}\right) \chi+\left(i^{*} f_{2}\right) \vartheta_{1}-\left(i^{*} L_{X_{f_{2}}} f_{1}\right) \chi-\left(i^{*} f_{1}\right) \vartheta_{2}\right. \\
& \left.+\left(i^{*} \omega\left(X_{f_{1}}, X_{f_{2}}\right)\right) \chi\right]=-\int_{L_{0}}\left(h_{1} \vartheta_{2}-h_{2} \vartheta_{1}\right)=-\Omega_{[(i, \chi)]}\left(\xi_{1}, \xi_{2}\right) .
\end{aligned}
$$

Nondegeneracy then follows from the fact that the $h$ 's and $\vartheta$ 's are independent of one another.

\section{Momentum weighted metrics}

In this section we apply the kinematic approach of the last section to the space of pseudoRiemannian metrics of fixed signature. We obtain a cotangent bundle and identify a Poisson algebra of functions on this space. These functions are Hamiltonians for Hamiltonian vector fields. In the particular case of Lorentzian metrics on a fixed 4-dimensional manifold, this gives some ingredients for a possible geometric quantization of gravity in a vacuum. 


\section{Convention}

From now on $M$ will no longer necessarily be symplectic. Instead we will simply assume that $M$ is a finite dimensional manifold.

\section{Notation}

Let $\operatorname{Met}^{q}(M)$ denote the set of pseudo Riemannian metrics on $M$ of signature $q$ and $\Gamma_{c}\left(S^{2} T^{*} M\right)$ the set of compactly supported symmetric two tensors on $M$.

Then $\operatorname{Met}^{q}(M)$ is a smooth manifold modeled on the space $\Gamma_{c}\left(S^{2} T^{*} M\right)$ (see Theorem 45.13 in [9]). By identifying such metrics with their graphs in $\Gamma\left(L\left(T M, T^{*} M\right)\right)$, we can view $\operatorname{Met}^{q}(M)$ as a collection of submanifolds. If as in Section 11 we view $\operatorname{Met}^{q}(M)$ kinematically as the set of possible configurations of a submanifold moving in $\Gamma\left(L\left(T M, T^{*} M\right)\right)$, then by analogy we should be able to describe the "conjugate momenta" in terms of some kind of "weightings" on the submanifolds.

To determine what these weightings should be, we first note that since the submanifolds in $\Gamma\left(L\left(T M, T^{*} M\right)\right)$ corresponding to pseudo-Riemannian metrics in $\operatorname{Met}^{q}(M)$ are graphs, any sections of bundles over such manifolds can be obtained as pullups of sections over corresponding bundles on the base $M$. Second, the weightings should give linear functionals on each tangent space to $\operatorname{Met}^{q}(M)$ and be in 1-1 correspondence with $\Gamma_{c}\left(S^{2} T^{*} M\right)$. This motivates the following definition:

Definition 17. A momentum weighting of a pseudo Riemannian metric $g \in \operatorname{Met}^{q}(M)$ of signature $q$ is a symmetric two tensor $h \in \Gamma_{c}\left(S^{2} T^{*} M\right)$. Pairs $(g, h)$ will be called momentum weighted metrics.

\section{Notation}

Let $\operatorname{Met}_{m w}^{q}(M)$ denote the set of momentum weighted metrics of signature $q$. That is,

$$
\operatorname{Met}_{m w}^{q}(M):=\operatorname{Met}^{q}(M) \times \Gamma_{c}\left(S^{2} T^{*} M\right) .
$$

We will show that $\operatorname{Met}_{m w}^{q}(M)$ is the appropriate phase space for $\operatorname{Met}^{q}(M)$. Define a 1-form $\Theta$ on $\operatorname{Met}_{m w}^{q}(M)$ via

$$
\Theta_{(g, h)}(k, l):=\int_{M} \operatorname{Tr}\left(g^{-1} k g^{-1} h\right) \mu(g),
$$

where $\operatorname{Tr}\left(g^{-1} k g^{-1} h\right)=g^{j r} k_{r q} g^{q p} h_{p j}, \mu(g)=\sqrt{|\operatorname{det} g|}\left|d x_{1} \wedge \cdots \wedge d x_{n}\right|$.

We will now explicitly calculate the exterior derivative of $\Theta$. Given a pair of tangent vectors $\left(k_{1}, l_{1}\right),\left(k_{2}, l_{2}\right) \in T_{(g, h)} \operatorname{Met}_{m w}^{q}(M)$, extend each $\left(k_{p}, l_{p}\right)$ to a vector field $\xi_{p}$ on $\operatorname{Met}_{m w}^{q}(M)$ satisfying $\xi_{p}\left(\left(g^{\prime}, h^{\prime}\right)\right)=\left(k_{p}, l_{p}\right)$ for all $\left(g^{\prime}, h^{\prime}\right)$ near $(g, h)$. Since as pointwise matrices,

$$
\begin{aligned}
& \left.\frac{d}{d t}\right|_{t=0}(g+t k)^{-1}=-g^{-1} k g^{-1}, \\
& \left.\frac{d}{d t}\right|_{t=0} \sqrt{|\operatorname{det}(g+t k)|}=\sqrt{|\operatorname{det} g|} \operatorname{Tr}\left(g^{-1} k\right),
\end{aligned}
$$


it follows that

$$
\begin{aligned}
d \Theta_{(g, h)}\left(\left(k_{1}, l_{1}\right),\left(k_{2}, l_{2}\right)\right)= & {\left[d \Theta\left(\xi_{1}, \xi_{2}\right)\right]_{(g, h)} } \\
= & \int_{M}\left[-\operatorname{Tr}\left(g^{-1} k_{1} g^{-1} k_{2} g^{-1} h\right)-\operatorname{Tr}\left(g^{-1} k_{2} g^{-1} k_{1} g^{-1} h\right)\right. \\
& \left.+\operatorname{Tr}\left(g^{-1} k_{2} g^{-1} h\right) \operatorname{Tr}\left(g^{-1} k_{1}\right)+\operatorname{Tr}\left(g^{-1} k_{2} g^{-1} l_{1}\right)\right] \mu(g)-(1 \leftrightarrow 2) \\
= & \int_{M}\left[\operatorname{Tr}\left(g^{-1} k_{2} g^{-1} h\right) \operatorname{Tr}\left(g^{-1} k_{1}\right)-\operatorname{Tr}\left(g^{-1} k_{1} g^{-1} h\right) \operatorname{Tr}\left(g^{-1} k_{2}\right)\right. \\
& \left.+\operatorname{Tr}\left(g^{-1} k_{2} g^{-1} l_{1}\right)-\operatorname{Tr}\left(g^{-1} k_{1} g^{-1} l_{2}\right)\right] \mu(g) .
\end{aligned}
$$

Theorem 9. The 2-form $\Omega:=-d \Theta$ defined by

$$
\begin{aligned}
\Omega_{(g, h)}\left(\xi_{1}, \xi_{2}\right)= & \int_{M}\left[\operatorname{Tr}\left(g^{-1} k_{1} g^{-1} h\right) \operatorname{Tr}\left(g^{-1} k_{2}\right)-\operatorname{Tr}\left(g^{-1} k_{2} g^{-1} h\right) \operatorname{Tr}\left(g^{-1} k_{1}\right)\right. \\
& \left.+\operatorname{Tr}\left(g^{-1} k_{1} g^{-1} l_{2}\right)-\operatorname{Tr}\left(g^{-1} k_{2} g^{-1} l_{1}\right)\right] \mu(g)
\end{aligned}
$$

defines an exact symplectic structure on $\operatorname{Met}_{m w}^{q}(M)$.

Proof. If $k_{1}$ is nonzero, then by taking $k_{2}=0$ the expression for $\Omega_{(g, h)}\left(\xi_{1}, \xi_{2}\right)$ reduces to $\int_{M} \operatorname{Tr}\left(g^{-1} k_{1} g^{-1} l_{2}\right) \mu(g)$. Since

$$
G_{g}\left(k_{1}, k_{2}\right)=\int_{M} \operatorname{Tr}\left(g^{-1} k_{1} g^{-1} k_{2}\right) \mu(g)
$$

is weakly nondegenerate (see Lemma 45.3 in [9]), $l_{2}$ can be chosen so that the integral does not vanish.

If $k_{1}=0$, then we are left with $-\int_{M} \operatorname{Tr}\left(g^{-1} k_{2} g^{-1} l_{1}\right) \mu(g)$ which means we can choose $k_{2}$ so that the integral does not vanish for the same reason.

\section{Notation}

For every section $r \in \Gamma_{c}\left(S^{2} T^{*} M\right)$, define the function

$$
F_{r}: \operatorname{Met}_{m w}^{q}(M) \rightarrow \mathbb{R}, \quad F_{r}(g, h):=\int_{M} \operatorname{Tr}\left(g^{-1} r g^{-1} h\right) \mu(g) .
$$

Proposition 18. For every $r \in \Gamma_{c}\left(S^{2} T^{*} M\right)$, the vector field $\xi_{F_{r}}$ defined by

$$
\xi_{F_{r}}(g, h):=\left(r, r g^{-1} h+h g^{-1} r-\operatorname{Tr}\left(g^{-1} r\right) \cdot h\right)
$$

is a Hamiltonian vector field on $\operatorname{Met}_{m w}^{q}(M)$ corresponding to the Hamiltonian function $F_{r}$, i.e. $\imath\left(\xi_{F_{r}}\right) \Omega=d F_{r}$.

Proof. Given $(k, l) \in T_{(g, h)} \operatorname{Met}_{m w}^{q}(M)$, extend $(k, l)$ constantly to a vector field $\xi$ on $\operatorname{Met}_{m w}^{q}(M)$ satisfying $\xi\left(g^{\prime}, h^{\prime}\right)=(k, l)$. Then

$$
\begin{aligned}
\left(d F_{r}\right)_{(g, h)}(\xi)= & \int_{M}\left[-\operatorname{Tr}\left(g^{-1} k g^{-1} r g^{-1} h\right)-\operatorname{Tr}\left(g^{-1} r g^{-1} k g^{-1} h\right)\right. \\
& \left.+\operatorname{Tr}\left(g^{-1} r g^{-1} h\right) \operatorname{Tr}\left(g^{-1} k\right)+\operatorname{Tr}\left(g^{-1} r g^{-1} l\right)\right] \mu(g)=\Omega_{(g, h)}\left(\xi_{F_{r}}, \xi\right) .
\end{aligned}
$$




\section{Notation}

The manifold topology on $\operatorname{Met}_{m w}^{q}(M)$ is finer than the trace of the Whitney $C^{\infty}$-topology on $\Gamma\left(L\left(T M, T^{*} M\right)\right)$ (see Section 45.1 in [9]). Let $C_{\int}^{\infty}\left(\operatorname{Met}_{m w}^{q}(M)\right)$ denote the set of functions $F: \operatorname{Met}_{m w}^{q}(M) \rightarrow \mathbb{R}$ such that for every $g_{0} \in \operatorname{Met}_{m w}^{q}(M)$ there exists a neighbourhood $U$ of $g_{0}$, a map $A: \mathbb{R}^{n} \rightarrow \mathbb{R}$, and sections $r_{1}, \ldots, r_{n} \in \Gamma_{c}\left(S^{2} T^{*} M\right)$ so that

$$
F(g)=A\left(\int_{M} \operatorname{Tr}\left(g^{-1} r_{1} g^{-1} h\right) \mu(g), \ldots, \int_{M} \operatorname{Tr}\left(g^{-1} r_{n} g^{-1} h\right) \mu(g)\right)
$$

for all $g \in U$.

Remark 18. The algebra $C_{\int}^{\infty}\left(\operatorname{Met}_{m w}^{q}(M)\right)$ contains the constant functions.

By an argument similar to that in Proposition 16, we have the following proposition:

Proposition 19. For every $F \in C_{\int}^{\infty}\left(\operatorname{Met}_{m w}^{q}(M)\right)$, the local assignments given by $v_{F}(g):=$ $\sum_{j=1}^{n} \frac{\partial A}{\partial y_{j}} \cdot X_{F_{r_{j}}}(g)$ on each neighbourhood $U$ define a unique vector field $v_{F}$ on $\operatorname{Met}_{m w}^{q}(M)$ satisfying $d F=\imath\left(v_{F}\right) \Omega$.

It follows that $\{F, G\}:=-\Omega\left(v_{F}, v_{G}\right)$ defines a Poisson bracket on $\operatorname{Met}_{m w}^{q}(M)$.

\section{Acknowledgements}

I thank Eckhard Meinrenken for suggesting this project and Yael Karshon who joint supervised this work as part of the author's PhD thesis. I also thank Boris Khesin for his many helpful suggestions towards improving both the content and exposition of this paper.

\section{References}

[1] Binz E., Fischer H.R., The manifold of embeddings of a closed manifold, in Differential Geometric Methods in Mathematical Physics (Proc. Internat. Conf., Tech. Univ. Clausthal, Clausthal-Zellerfeld, 1978), Editor H.D. Doebner, Lecture Notes in Phys., Vol. 139, Springer, Berlin - New York, 1981, 310-329.

[2] Cushman R., Sniatycki J., Differential structure of orbit spaces, Canad. J. Math. 53 (2001), 715-755.

[3] Donaldson S., Moment maps and diffeomorphisms, Asian J. Math. 3 (1999), 1-15.

[4] Donaldson S., Scalar curvature and projective embeddings. I, J. Differential Geom. 59 (2001), 479-522.

[5] Ebin D., The manifold of Riemannian metrics, in Proceedings of Symposia in Pure Mathematics (1968, Berkeley), Global Analysis, American Mathematical Society, Providence, RI, 1970, 11-40.

[6] Frölicher A., Kriegl A., Linear spaces and differentiation theory, Pure and Applied Mathematics, John Wiley \& Sons, Chichester, 1988.

[7] Hamilton R.S., The inverse function theorem of Nash and Moser, Bull. Amer. Math. Soc. (N.S.) 7 (1982), $65-222$.

[8] Khesin B., Lee P., Poisson geometry and first integrals of geostrophic equations, Phys. D 237 (2008), 2072-2077, arXiv:0802.4439.

[9] Kriegl A., Michor P.W., The convenient setting of global analysis, Mathematical Surveys and Monographs, Vol. 53, American Mathematical Society, Providence, RI, 1997.

[10] Moser J., On the volume elements on a manifold, Trans. Amer. Math. Soc. 120 (1965), 280-296.

[11] Ortega J.P., Ratiu T.S., The optimal momentum map, in Geometry, Mechanics, and Dynamics, Springer, New York, 2002, 329-362.

[12] Polterovich L., The geometry of the group of symplectic diffeomorphisms, Lectures in Mathematics ETH Zürich, Birkhäuser Verlag, Basel, 2001.

[13] Sikorski R., Introduction to differential geometry, Biblioteka Matematyczna, Tom 42, PWN, Warsaw, 1972 (in Polish). 
[14] Smolentsev N.K., Natural weak Riemannian structures in the space of Riemannian metrics, Sibirsk. Mat. Zh. 35 (1994), 439-445 (English transl.: Siberian Math. J. 35 (1994), 396-402).

[15] Souriau J.M., Groupes différentiels, in Differential Geometrical Methods in Mathematical Physics (Proc. Conf., Aix-en-Provence/Salamanca, 1979), Lecture Notes in Math., Vol. 836, Springer, Berlin - New York, 1980, 91-128.

[16] Stefan P., Accessibility and foliations with singularities, Bull. Amer. Math. Soc. 80 (1974), 1142-1145.

[17] Stefan P., Accessible sets, orbits, and foliations with singularities, Proc. London Math. Soc. (3) 29 (1974), 699-713.

[18] Sussmann H.J., Orbits of families of vector fields and integrability of systems with singularities, Bull. Amer. Math. Soc. 79 (1973), 197-199.

[19] Sussmann H.J., Orbits of families of vector fields and integrability of distributions, Trans. Amer. Math. Soc. 180 (1973), 171-188.

[20] Warner F.W., Foundations of differentiable manifolds and Lie groups, Graduate Texts in Mathematics, Vol. 94, Springer-Verlag, New York - Berlin, 1983.

[21] Weinstein A., Symplectic manifolds and their Lagrangian submanifolds, Adv. Math. 6 (1971), 329-346.

[22] Weinstein A., Neighborhood classification of isotropic embeddings, J. Differential Geom. 16 (1981), 125-128.

[23] Weinstein A., Connections of Berry and Hannay type for moving Lagrangian submanifolds, Adv. Math. 82 (1990), 133-159. 